$$
\text { for }
$$

\title{
Deoxyfluorination of Carboxylic Acids with CpFluor: Access to Acyl Fluorides and Amides
}

\author{
Xiu Wang, Fei Wang, Fengfeng Huang, Chuanfa Ni, and Jinbo Hu* \\ Key Laboratory of Organofluorine Chemistry, Center for Excellence in Molecular Synthesis, Shanghai \\ Institute of Organic Chemistry, University of Chinese Academy of Sciences, Chinese Academy of Sciences, \\ 345 Ling-Ling Road, Shanghai 200032, China. \\ Email: jinbohu@sioc.ac.cn
}

\section{Table of Contents}

$\begin{array}{ll}\text { 1. General Information } & S 2\end{array}$

2. Synthesis of 3,3-Difluoro-1,2-diphenylcyclopropene (CpFluor 2) S3

3. Procedures for Deoxyfluorination of Carboxylic Acids $\quad S 4$

4. Procedures for One-Pot Deoxyfluorination/Amination of Carboxylic Acids $\quad$ S15

5. Procedures for Two-Step Acyl Fluorides Synthesis via in-situ Formed CpFluor $\quad$ S19

6. Copies of NMR Charts for Products $\quad S 21$

7. References $\quad S 52$ 


\section{General Information}

Unless otherwise noted, all the reactions were carried out under a nitrogen atmosphere using standard Schlenk techniques. Glassware was dried in an oven $\left(100{ }^{\circ} \mathrm{C}\right)$ and heated under reduced pressure prior to use. Solvents were employed as eluents for all other routine operation, compounds $\mathbf{1}$ and dehydrated solvents were purchased from commercial suppliers and employed without any further purification. ${ }^{1} \mathrm{H}$ NMR spectra were recorded at $400 \mathrm{MHz}$ or $300 \mathrm{MHz} .{ }^{13} \mathrm{C}$ NMR spectra were recorded at $101 \mathrm{MHz} .{ }^{19} \mathrm{~F}$ NMR spectra were recorded at $396 \mathrm{MHz}$ or $282 \mathrm{MHz} .{ }^{1} \mathrm{H}$ NMR chemical shifts were determined relative to internal $\left(\mathrm{CH}_{3}\right)_{4} \mathrm{Si}$ (TMS) at $\delta 0.00 \mathrm{ppm}$ or to the signal of the residual protonated solvent: $\mathrm{CDCl}_{3}$ at $\delta 7.26$ ppm, ${ }^{13} \mathrm{C}$ NMR chemical shifts were determined relative to the signal of the solvent: $\mathrm{CDCl}_{3}$ at $\delta 77.16 \mathrm{ppm}$.

${ }^{19} \mathrm{~F}$ NMR chemical shifts were determined relative to internal $\mathrm{CFCl}_{3}$ at $\delta 0.00 \mathrm{ppm}$. Data for ${ }^{1} \mathrm{H},{ }^{13} \mathrm{C},{ }^{19} \mathrm{~F}$ NMR were recorded as follows: chemical shift $(\delta, \mathrm{ppm})$, multiplicity $(\mathrm{s}=$ singlet, $\mathrm{d}=$ doublet, $\mathrm{t}=$ triplet, $\mathrm{m}$ $=$ multiplet, $\mathrm{q}=$ quartet, $\mathrm{dd}=$ doublet of doublets, $\mathrm{dt}=$ doublet of triplets, $\mathrm{td}=$ triplet of doublets, $\mathrm{br}=$ broad). High-resolution mass data were recorded on a high-resolution mass spectrometer in the EI or ESI mode. 


\section{Synthesis of 3,3-Difluoro-1,2-diphenylcyclopropene (CpFluor, 2)}

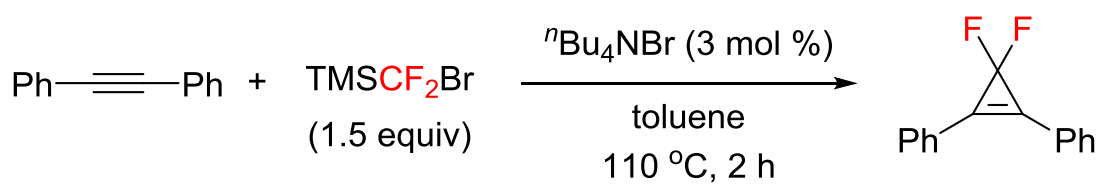

To an oven-dried sealed tube charged with a magnetic stirrer bar, were successively added 1,2-diphenylethyne $(10 \mathrm{mmol}, 1.78 \mathrm{~g}),{ }^{n} \mathrm{Bu}_{4} \mathrm{NBr}(0.3 \mathrm{mmol}, 3 \mathrm{~mol} \%, 96.7 \mathrm{mg})$, toluene $(10 \mathrm{~mL})$ and $\mathrm{TMSCF}_{2} \mathrm{Br}(15 \mathrm{mmol}, 1.5$ equiv, $3.05 \mathrm{~g})$. The reaction mixture was immersed into $110{ }^{\circ} \mathrm{C}$ oil bath and stirred for $2 \mathrm{~h}$. After the reaction was completed, the reaction mixture was cooled to room temperature and quenched with $60 \mathrm{~mL}$ diethyl ether and $10 \mathrm{~mL} \mathrm{Et}{ }_{3} \mathrm{~N}$, extracted with $5 \%$ of $\mathrm{Na}_{2} \mathrm{CO}_{3}(30 \mathrm{~mL} \times 3)$. The combined organic layer was dried over $\mathrm{K}_{2} \mathrm{CO}_{3}$. The organic solution was then concentrated to dryness and the residue was recrystallized by hexane/Et ${ }_{3} \mathrm{~N}=10 / 1$ to afford 3,3-difluoro-1,2-diphenylcyclopropene (CpFluor 2) in 53\% (1.2 g).

\section{(3,3-Difluorocycloprop-1-ene-1,2-diyl)dibenzene (2)}

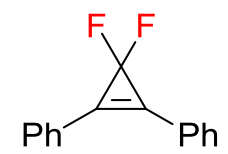

Performed on $10 \mathrm{mmol}$ scale, recrystallization by hexane/Et $\mathrm{t}_{3} \mathrm{~N}=10 / 1$ to give $2(1.2 \mathrm{~g}, 53 \%$ yield $)$.

White solid. ${ }^{1} \mathbf{H}$ NMR $\left(400 \mathrm{MHz}, \mathrm{CDCl}_{3}\right) \delta$ 7.82-7.75 (m, 4H), 7.56-7.48 (m, 6H).

${ }^{19}$ F NMR $\left(376 \mathrm{MHz}, \mathrm{CDCl}_{3}\right) \delta-112.2(\mathrm{~s}, 1 \mathrm{~F})$.

MS (EI, m/z): $228\left(\mathbf{M}^{+}\right)$.

The characterization data are consistent with the previous report. ${ }^{1}$ 


\section{Procedures for Deoxyfluorination of Carboxylic Acids}<smiles>[R]C(=O)O</smiles>

1<smiles>FC1(F)C(P)=C1c1ccccc1</smiles>

2

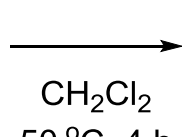

$50{ }^{\circ} \mathrm{C}, 4 \mathrm{~h}$<smiles>[R]C(=O)F</smiles>

3

To an oven-dried sealed tube charged with a magnetic stirrer bar, were successively added carboxylic acid $\mathbf{1}$ (0.5 mmol), CpFluor 2 (0.8 mmol, 1.6 equiv, $183 \mathrm{mg}$ ), and $\mathrm{CH}_{2} \mathrm{Cl}_{2}(2.5 \mathrm{~mL})$. The reaction mixture was immersed into $50{ }^{\circ} \mathrm{C}$ oil bath and stirred for $4 \mathrm{~h}$. The solution was then cooled to room temperature, and the mixture was extracted with $\mathrm{CH}_{2} \mathrm{Cl}_{2}$ and $\mathrm{H}_{2} \mathrm{O}$. The obtained organic layer was dried over $\mathrm{MgSO}_{4}$, then concentrated under vacuum. The residue was purified by flash chromatography with petroleum ether/ethyl acetate as eluent to afford the corresponding acyl fluorides $\mathbf{3}$.

\section{3-Methylbenzoyl fluoride (3a)}<smiles>Cc1cccc(C(=O)F)c1</smiles>

Performed on $0.5 \mathrm{mmol}$ scale, eluted with petroleum ether/ethyl acetate $=20 / 1$ to give $\mathbf{3 a}(48.3 \mathrm{mg}, 70 \%$ yield).

Colorless oil. ${ }^{1} \mathbf{H}$ NMR $\left(300 \mathrm{MHz}, \mathrm{CDCl}_{3}\right) \delta$ 8.05-7.77 (m, 2H), 7.45 (dt, $\left.J=26.6,7.7 \mathrm{~Hz}, 2 \mathrm{H}\right), 2.43$ (s, $3 \mathrm{H})$.

${ }^{19}$ F NMR $\left(282 \mathrm{MHz}, \mathrm{CDCl}_{3}\right) \delta 18.5(\mathrm{~s}, 1 \mathrm{~F})$.

MS (EI, m/z): $138\left(\mathbf{M}^{+}\right)$.

The characterization data are consistent with the previous report. ${ }^{2}$

\section{2-Naphthoyl fluoride (3b)}<smiles>O=C(F)c1ccc2ccccc2c1</smiles>

Performed on $0.5 \mathrm{mmol}$ scale, eluted with petroleum ether/ethyl acetate $=20 / 1$ to give $\mathbf{3 b}(73.2 \mathrm{mg}, 84 \%$ yield). 
White solid. ${ }^{1}$ H NMR $\left(400 \mathrm{MHz}, \mathrm{CDCl}_{3}\right) \delta 8.62(\mathrm{~s}, 1 \mathrm{H}), 7.95(\mathrm{dt}, J=23.8,9.1 \mathrm{~Hz}, 4 \mathrm{H}), 7.68(\mathrm{td}, J=8.2$, 6.9, $1.4 \mathrm{~Hz}, 1 \mathrm{H}), 7.61(\mathrm{td}, J=8.2,6.9,1.3 \mathrm{~Hz}, 1 \mathrm{H})$.

${ }^{19}$ F NMR $\left(376 \mathrm{MHz}, \mathrm{CDCl}_{3}\right) \delta 18.1(\mathrm{~s}, 1 \mathrm{~F})$.

MS (EI, m/z): $174\left(\mathbf{M}^{+}\right)$.

The characterization data are consistent with the previous report. ${ }^{3}$

\section{1-Naphthoyl fluoride (3c)}<smiles>O=C(F)c1cccc2ccccc12</smiles>

Performed on $0.5 \mathrm{mmol}$ scale, eluted with petroleum ether/ethyl acetate $=20 / 1$ to give $3 \mathbf{c}(61.0 \mathrm{mg}, 70 \%$ yield).

White solid. ${ }^{1} \mathbf{H}$ NMR $\left(400 \mathrm{MHz}, \mathrm{CDCl}_{3}\right) \delta 9.01(\mathrm{~d}, J=8.7 \mathrm{~Hz}, 1 \mathrm{H}), 8.34(\mathrm{~d}, J=7.3 \mathrm{~Hz}, 1 \mathrm{H}), 8.16(\mathrm{~d}, J=$ $8.2 \mathrm{~Hz}, 1 \mathrm{H}), 7.93(\mathrm{~d}, J=8.2 \mathrm{~Hz}, 1 \mathrm{H}), 7.71(\mathrm{td}, J=8.5,6.8,1.5 \mathrm{~Hz}, 1 \mathrm{H}), 7.58(\mathrm{dt}, J=27.2,8.0 \mathrm{~Hz}, 2 \mathrm{H})$.

${ }^{19}$ F NMR $\left(376 \mathrm{MHz}, \mathrm{CDCl}_{3}\right) \delta 29.9(\mathrm{~s}, 1 \mathrm{~F})$

MS (EI, m/z): $174\left(\mathbf{M}^{+}\right)$.

The characterization data are consistent with the previous report. ${ }^{3}$

\section{4-Tert-butylbenzoyl fluoride (3e)}<smiles>CC(C)(C)c1ccc(C(=O)F)cc1</smiles>

Performed on $0.5 \mathrm{mmol}$ scale, eluted with petroleum ether/ethyl acetate $=20 / 1$ to give $3 \mathbf{e}(63.0 \mathrm{mg}, 70 \%$ yield).

White solid. ${ }^{1} \mathbf{H}$ NMR $\left(300 \mathrm{MHz}, \mathrm{CDCl}_{3}\right) \delta 7.97(\mathrm{dd}, J=8.6,2.3 \mathrm{~Hz}, 2 \mathrm{H}), 7.54(\mathrm{~d}, J=8.4 \mathrm{~Hz}, 2 \mathrm{H}), 1.36$ (s, 9H).

${ }^{19}$ F NMR $\left(282 \mathrm{MHz}, \mathrm{CDCl}_{3}\right) \delta 18.2(\mathrm{~s}, 1 \mathrm{~F})$.

MS (EI, m/z): $180\left(\mathbf{M}^{+}\right)$.

The characterization data are consistent with the previous report. ${ }^{4}$ 


\section{4-Methoxybenzoyl fluoride (3f)}<smiles>COc1ccc(C(=O)F)cc1</smiles>

Performed on $0.5 \mathrm{mmol}$ scale, eluted with petroleum ether/ethyl acetate $=20 / 1$ to give $\mathbf{3 f}(64.7 \mathrm{mg}, 84 \%$ yield).

Colorless oil. ${ }^{1} \mathbf{H}$ NMR $\left(300 \mathrm{MHz}, \mathrm{CDCl}_{3}\right) \delta 7.99(\mathrm{~d}, J=7.7 \mathrm{~Hz}, 2 \mathrm{H}), 7.03-6.94(\mathrm{~m}, 2 \mathrm{H}), 3.90(\mathrm{~s}, 3 \mathrm{H})$.

${ }^{19}$ F NMR $\left(282 \mathrm{MHz}, \mathrm{CDCl}_{3}\right) \delta 16.2(\mathrm{~s}, 1 \mathrm{~F})$.

MS (EI, m/z): $154\left(\mathrm{M}^{+}\right)$.

The characterization data are consistent with the previous report. ${ }^{2}$

\section{Benzo $[d][1,3]$ dioxole-5-carbonyl fluoride $(3 \mathrm{~g})$}<smiles>O=C(F)c1ccc2c(c1)OCO2</smiles>

Performed on $0.5 \mathrm{mmol}$ scale, eluted with petroleum ether/ethyl acetate $=20 / 1$ to give $\mathbf{3 g}(68.9 \mathrm{mg}, 82 \%$ yield).

White solid. ${ }^{1} \mathbf{H}$ NMR $\left(400 \mathrm{MHz}, \mathrm{CDCl}_{3}\right) \delta 7.65(\mathrm{~d}, J=8.2 \mathrm{~Hz}, 1 \mathrm{H}), 7.39(\mathrm{~s}, 1 \mathrm{H}), 6.89(\mathrm{~d}, J=8.4 \mathrm{~Hz}, 1 \mathrm{H})$, $6.09(\mathrm{~s}, 2 \mathrm{H})$

${ }^{19}$ F NMR $\left(376 \mathrm{MHz}, \mathrm{CDCl}_{3}\right) \delta 16.3(\mathrm{~s}, 1 \mathrm{~F})$.

MS (EI, m/z): $168\left(\mathrm{M}^{+}\right)$.

The characterization data are consistent with the previous report. ${ }^{3}$

\section{4-Chlorobenzoyl fluoride $(3 \mathrm{~h})$}<smiles>O=C(F)c1ccc(Cl)cc1</smiles>

Performed on $0.5 \mathrm{mmol}$ scale, eluted with petroleum ether/ethyl acetate $=20 / 1$ to give $\mathbf{3 h}(60.8 \mathrm{mg}, 77 \%$ yield).

White solid. ${ }^{1} \mathbf{H}$ NMR $\left(300 \mathrm{MHz}, \mathrm{CDCl}_{3}\right) \delta 7.99(\mathrm{~d}, J=8.5 \mathrm{~Hz}, 2 \mathrm{H}), 7.52(\mathrm{~d}, J=7.8 \mathrm{~Hz}, 2 \mathrm{H})$.

${ }^{19}$ F NMR $\left(282 \mathrm{MHz}, \mathrm{CDCl}_{3}\right) \delta 18.6(\mathrm{~s}, 1 \mathrm{~F})$ 
MS (EI, m/z): $158\left(\mathrm{M}^{+}\right)$.

The characterization data are consistent with the previous report. ${ }^{2}$

\section{4-Bromobenzoyl fluoride (3i)}<smiles>O=C(F)c1ccc(Br)cc1</smiles>

Performed on $0.5 \mathrm{mmol}$ scale, eluted with petroleum ether/ethyl acetate $=20 / 1$ to give $3 \mathbf{i}(78.2 \mathrm{mg}, 77 \%$ yield).

White solid. ${ }^{1} \mathbf{H}$ NMR $\left(300 \mathrm{MHz}, \mathrm{CDCl}_{3}\right) \delta 7.91(\mathrm{~d}, J=7.3 \mathrm{~Hz}, 2 \mathrm{H}), 7.69(\mathrm{~d}, J=8.1 \mathrm{~Hz}, 2 \mathrm{H})$.

${ }^{19}$ F NMR $\left(282 \mathrm{MHz}, \mathrm{CDCl}_{3}\right) \delta 18.7(\mathrm{~s}, 1 \mathrm{~F})$.

MS (EI, m/z): $202\left(\mathbf{M}^{+}\right)$.

The characterization data are consistent with the previous report. ${ }^{2}$

\section{4-(Bromomethyl)benzoyl fluoride $(3 \mathbf{j})$}<smiles>O=C(F)c1ccc(CBr)cc1</smiles>

Performed on $0.5 \mathrm{mmol}$ scale, eluted with petroleum ether/ethyl acetate $=20 / 1$ to give $\mathbf{3 j}$ ( $79.2 \mathrm{mg}, 73 \%$ yield).

White solid. m.p. 90-91 ${ }^{\circ} \mathrm{C} .{ }^{1} \mathbf{H}$ NMR $\left(300 \mathrm{MHz}, \mathrm{CDCl}_{3}\right) \delta 8.03(\mathrm{dd}, J=8.4,1.9 \mathrm{~Hz}, 2 \mathrm{H}), 7.55(\mathrm{~d}, J=8.3$ $\mathrm{Hz}, 2 \mathrm{H}), 4.51(\mathrm{~d}, J=1.9 \mathrm{~Hz}, 2 \mathrm{H})$.

${ }^{19}$ F NMR $\left(282 \mathrm{MHz}, \mathrm{CDCl}_{3}\right) \delta 33.0(\mathrm{~s}, 1 \mathrm{~F})$.

${ }^{13} \mathbf{C ~ N M R}\left(101 \mathrm{MHz}, \mathrm{CDCl}_{3}\right) \delta 156.9\left(\mathrm{~d},{ }^{1} J_{\mathrm{CF}}=344.2 \mathrm{~Hz}\right), 145.5,132.0\left(\mathrm{~d},{ }^{3} J_{\mathrm{CF}}=3.9 \mathrm{~Hz}\right), 129.8,124.9(\mathrm{~d}$, $\left.{ }^{2} J_{\mathrm{CF}}=61.4 \mathrm{~Hz}\right), 31.6$.

MS (EI, m/z): $206\left(\mathrm{M}^{+}\right)$.

HRMS (EI) m/z: [M] $]^{+}$Calcd. for $\mathrm{C}_{8} \mathrm{H}_{6} \mathrm{BrFO}$ : 215.9581; Found: 215.9584.

IR (film): 3060, 3036, 1808, 1605, 1578, 1417, 1259, 1200, 1176, 1032, 1012, 858, 825, 764, 736, 691, 608, $598,500,472 \mathrm{~cm}^{-1}$. 


\section{4-Nitrobenzoyl fluoride (3k)}<smiles>O=C(F)c1ccc([N+](=O)[O-])cc1</smiles>

Performed on $0.5 \mathrm{mmol}$ scale, eluted with petroleum ether/ethyl acetate $=20 / 1$ to give $3 \mathbf{k}(60.0 \mathrm{mg}, 71 \%$ yield).

White solid. ${ }^{1} \mathbf{H}$ NMR $\left(300 \mathrm{MHz}, \mathrm{CDCl}_{3}\right) \delta 8.39(\mathrm{~d}, J=8.5 \mathrm{~Hz}, 2 \mathrm{H}), 8.26(\mathrm{~d}, J=8.7 \mathrm{~Hz}, 2 \mathrm{H})$.

${ }^{19}$ F NMR $\left(282 \mathrm{MHz}, \mathrm{CDCl}_{3}\right) \delta 21.6(\mathrm{~s}, 1 \mathrm{~F})$.

MS (EI, m/z): $169\left(\mathrm{M}^{+}\right)$.

The characterization data are consistent with the previous report. ${ }^{5}$

\section{3-Nitrobenzoyl fluoride (31)}<smiles>O=C(F)c1cccc([N+](=O)[O-])c1</smiles>

Performed on $0.5 \mathrm{mmol}$ scale, eluted with petroleum ether/ethyl acetate $=20 / 1$ to give $3 \mathbf{3}(72.7 \mathrm{mg}, 86 \%$ yield).

White solid. ${ }^{1} \mathbf{H}$ NMR $\left(300 \mathrm{MHz}, \mathrm{CDCl}_{3}\right) \delta 8.89(\mathrm{~s}, 1 \mathrm{H}), 8.57(\mathrm{~d}, J=8.5 \mathrm{~Hz}, 1 \mathrm{H}), 8.39(\mathrm{~d}, J=7.8 \mathrm{~Hz}, 1 \mathrm{H})$, $7.79(\mathrm{t}, J=8.0 \mathrm{~Hz}, 1 \mathrm{H})$.

${ }^{19}$ F NMR $\left(282 \mathrm{MHz}, \mathrm{CDCl}_{3}\right) \delta 20.4(\mathrm{~s}, 1 \mathrm{~F})$.

MS (EI, m/z): $169\left(\mathrm{M}^{+}\right)$.

The characterization data are consistent with the previous report. ${ }^{6}$

\section{3-Chlorobenzoyl fluoride (3m)}<smiles>O=C(F)c1cccc(Cl)c1</smiles>

Performed on $0.5 \mathrm{mmol}$ scale, eluted with petroleum ether/ethyl acetate $=20 / 1$ to give $\mathbf{3 m}(58.7 \mathrm{mg}, 74 \%$ yield).

White solid. ${ }^{1} \mathbf{H}$ NMR $\left(300 \mathrm{MHz}, \mathrm{CDCl}_{3}\right) \delta 8.03(\mathrm{~s}, 1 \mathrm{H}), 7.95(\mathrm{~d}, J=8.1 \mathrm{~Hz}, 1 \mathrm{H}), 7.69(\mathrm{~d}, J=7.9 \mathrm{~Hz}, 1 \mathrm{H})$, $7.49(\mathrm{dt}, J=8.0,3.2 \mathrm{~Hz}, 1 \mathrm{H})$. 
${ }^{19}$ F NMR $\left(282 \mathrm{MHz}, \mathrm{CDCl}_{3}\right) \delta 19.4(\mathrm{~s}, 1 \mathrm{~F})$.

MS (EI, m/z): $158\left(\mathrm{M}^{+}\right)$.

The characterization data are consistent with the previous report. ${ }^{7}$

\section{2-Methoxybenzoyl fluoride (3n)}<smiles>COc1ccccc1C(=O)F</smiles>

Performed on $0.5 \mathrm{mmol}$ scale, eluted with petroleum ether/ethyl acetate $=20 / 1$ to give $3 \mathbf{n}(60.1 \mathrm{mg}, 78 \%$ yield).

Colorless oil. ${ }^{1} \mathbf{H}$ NMR $\left(300 \mathrm{MHz}, \mathrm{CDCl}_{3}\right) \delta 7.93(\mathrm{~d}, J=8.1 \mathrm{~Hz}, 1 \mathrm{H}), 7.63(\mathrm{t}, J=7.9 \mathrm{~Hz}, 1 \mathrm{H}), 7.04(\mathrm{td}, J=$ 8.2, 3.4, $1.9 \mathrm{~Hz}, 2 \mathrm{H}), 3.96(\mathrm{~s}, 3 \mathrm{H})$.

${ }^{19}$ F NMR $\left(282 \mathrm{MHz}, \mathrm{CDCl}_{3}\right) \delta 32.0(\mathrm{~s}, 1 \mathrm{~F})$.

MS (EI, m/z): $154\left(\mathrm{M}^{+}\right)$.

The characterization data are consistent with the previous report. ${ }^{8}$

\section{2-Iodobenzoyl fluoride (3o)}<smiles>O=C(F)c1ccccc1I</smiles>

Performed on $0.5 \mathrm{mmol}$ scale, eluted with petroleum ether/ethyl acetate $=20 / 1$ to give $3 \mathbf{3}(97.5 \mathrm{mg}, 78 \%$ yield).

White solid. ${ }^{1} \mathbf{H}$ NMR $\left(300 \mathrm{MHz}, \mathrm{CDCl}_{3}\right) \delta 8.14(\mathrm{~d}, J=7.9 \mathrm{~Hz}, 1 \mathrm{H}), 8.03(\mathrm{~d}, J=7.8 \mathrm{~Hz}, 1 \mathrm{H}), 7.51(\mathrm{t}, J=$ $6.9 \mathrm{~Hz}, 1 \mathrm{H}), 7.35-7.25(\mathrm{~m}, 1 \mathrm{H})$.

${ }^{19}$ F NMR $\left(282 \mathrm{MHz}, \mathrm{CDCl}_{3}\right) \delta 28.9(\mathrm{~s}, 1 \mathrm{~F})$.

MS (EI, m/z): $259\left(\mathrm{M}^{+}\right)$.

The characterization data are consistent with the previous report. ${ }^{9}$

\section{5-Bromo-2-methylbenzoyl fluoride (3p)}


<smiles>Cc1ccc(Br)cc1C(=O)F</smiles>

Performed on $0.5 \mathrm{mmol}$ scale, eluted with petroleum ether/ethyl acetate $=20 / 1$ to give $\mathbf{3 p}(85.7 \mathrm{mg}, 79 \%$ yield).

White solid. m.p. $47-48{ }^{\circ} \mathrm{C} .{ }^{1} \mathbf{H}$ NMR $\left(300 \mathrm{MHz}, \mathrm{CDCl}_{3}\right) \delta 8.11(\mathrm{~s}, 1 \mathrm{H}), 7.66(\mathrm{dd}, J=8.2,2.1 \mathrm{~Hz}, 1 \mathrm{H})$, 7.27-7.20 (m, 1H), $2.60(\mathrm{~s}, 3 \mathrm{H})$.

${ }^{19}$ F NMR $\left(282 \mathrm{MHz}, \mathrm{CDCl}_{3}\right) \delta 30.0(\mathrm{~s}, 1 \mathrm{~F})$.

${ }^{13} \mathrm{C}$ NMR $\left(101 \mathrm{MHz}, \mathrm{CDCl}_{3}\right) \delta 155.5\left(\mathrm{~d},{ }^{1} J_{\mathrm{CF}}=346.5 \mathrm{~Hz}\right), 142.5,137.6,135.1,133.9,125.5\left(\mathrm{~d},{ }^{2} J_{\mathrm{CF}}=57.8\right.$ Hz), 119.8, 21.5.

MS (EI, m/z): $216\left(\mathrm{M}^{+}\right)$.

HRMS (EI) m/z: [M] $]^{+}$Calcd. for $\mathrm{C}_{8} \mathrm{H}_{6} \mathrm{BrFO}$ : 215.9581; Found: 215.9583.

IR (film): 3580, 3077, 2992, 2928, 2856, 1935, 1800, 1590, 1562, 1481, 1445, 1383, 1289, 1238, 1217 , $1094,1007,994,888,829,763,750,663,626,517,500 \mathrm{~cm}^{-1}$.

\section{5-Bromo-2-chlorobenzoyl fluoride $(3 q)$}<smiles>O=C(F)c1cc(Br)ccc1Cl</smiles>

Performed on $0.5 \mathrm{mmol}$ scale, eluted with petroleum ether/ethyl acetate $=20 / 1$ to give $\mathbf{3 q}(72.4 \mathrm{mg}, 61 \%$ yield).

White solid. m.p. $54-55{ }^{\circ} \mathrm{C} .{ }^{1} \mathbf{H}$ NMR $\left(300 \mathrm{MHz}, \mathrm{CDCl}_{3}\right) \delta 8.14(\mathrm{~s}, 1 \mathrm{H}), 7.70(\mathrm{~d}, J=8.5 \mathrm{~Hz}, 1 \mathrm{H}), 7.44(\mathrm{~d}, J$ $=8.6 \mathrm{~Hz}, 1 \mathrm{H})$

${ }^{19}$ F NMR $\left(376 \mathrm{MHz}, \mathrm{CDCl}_{3}\right) \delta 32.5(\mathrm{~s}, 1 \mathrm{~F})$.

${ }^{13} \mathbf{C ~ N M R}\left(101 \mathrm{MHz}, \mathrm{CDCl}_{3}\right) \delta 153.3\left(\mathrm{~d},{ }^{1} J_{\mathrm{CF}}=345.6 \mathrm{~Hz}\right), 138.4,136.2,135.9,133.4,125.2\left(\mathrm{~d},{ }^{2} J_{\mathrm{CF}}=62.5\right.$ $\mathrm{Hz}), 120.7$.

MS (EI, m/z): $236\left(\mathrm{M}^{+}\right)$.

HRMS (EI) m/z: [M] $]^{+}$Calcd. for $\mathrm{C}_{7} \mathrm{H}_{3} \mathrm{BrClFO}$ : 235.9034; Found: 235.9039.

IR (film): 3606, 3092, 1934, 1811, 1576, 1526, 1462, 1378, 1284, 1266, 1218, 1147, 1091, 1002, 896, 831, $761,677,651,507 \mathrm{~cm}^{-1}$. 


\section{2-Chloronicotinoyl fluoride (3r)}<smiles>O=C(F)c1cccnc1Cl</smiles>

Performed on $0.5 \mathrm{mmol}$ scale, eluted with petroleum ether/ethyl acetate $=20 / 1$ to give $\mathbf{3 r}(40.7 \mathrm{mg}, 51 \%$ yield).

White solid. Mp. $75-76{ }^{\circ} \mathrm{C} .{ }^{1} \mathbf{H}$ NMR $\left(300 \mathrm{MHz}, \mathrm{CDCl}_{3}\right) \delta 8.73-8.63(\mathrm{~m}, 1 \mathrm{H}), 8.35(\mathrm{~d}, J=7.8 \mathrm{~Hz}, 1 \mathrm{H})$, $7.45(\mathrm{dd}, J=7.9,4.9 \mathrm{~Hz}, 1 \mathrm{H})$.

${ }^{19}$ F NMR $\left(282 \mathrm{MHz}, \mathrm{CDCl}_{3}\right) \delta 32.9(\mathrm{~s}, 1 \mathrm{~F})$.

${ }^{13} \mathbf{C ~ N M R}\left(101 \mathrm{MHz}, \mathrm{CDCl}_{3}\right) \delta 155.4,154.4,152.3\left(\mathrm{~d},{ }^{1} J_{\mathrm{CF}}=82.2 \mathrm{~Hz}\right), 142.4,122.7,121.1\left(\mathrm{~d},{ }^{2} J_{\mathrm{CF}}=64.9\right.$ $\mathrm{Hz})$.

MS (EI, m/z): $159\left(\mathbf{M}^{+}\right)$.

HRMS (EI) m/z: $[\mathrm{M}]^{+}$Calcd. for $\mathrm{C}_{6} \mathrm{H}_{3} \mathrm{ClFNO}$ : 158.9882; Found: 158.9888.

IR (film): 3445, 3064, 1825, 1579, 1562, 1408, 1276, 1235, 1112, 996, 799, 760, 711, 700, 633, 535, 419 $\mathrm{cm}^{-1}$.

\section{Benzo $[b]$ thiophene-2-carbonyl fluoride (3s)}<smiles>O=C(F)c1cc2ccccc2s1</smiles>

Performed on $0.5 \mathrm{mmol}$ scale, eluted with petroleum ether/ethyl acetate $=20 / 1$ to give $3 \mathbf{s}(51.4 \mathrm{mg}, 57 \%$ yield).

White solid, ${ }^{1} \mathbf{H}$ NMR $\left(400 \mathrm{MHz}, \mathrm{CDCl}_{3}\right) \delta 8.19$ (s, 1H), $7.92(\mathrm{dd}, J=15.4,8.1 \mathrm{~Hz}, 2 \mathrm{H}), 7.55(\mathrm{td}, J=8.2$, 7.1, $1.3 \mathrm{~Hz}, 1 \mathrm{H}), 7.50-7.45(\mathrm{~m}, 1 \mathrm{H})$.

${ }^{19}$ F NMR $\left(376 \mathrm{MHz}, \mathrm{CDCl}_{3}\right) \delta 25.1(\mathrm{~s}, 1 \mathrm{~F})$.

MS (EI, m/z): $180\left(\mathrm{M}^{+}\right)$.

The characterization data are consistent with the previous report. ${ }^{10}$

\section{(E)-3-(Naphthalen-1-yl)acryloyl fluoride (3t)}


<smiles>O=C(F)/C=C/c1cccc2ccccc12</smiles>

Performed on $0.5 \mathrm{mmol}$ scale, eluted with petroleum ether/ethyl acetate $=20 / 1$ to give $3 \mathbf{t}(68.1 \mathrm{mg}, 68 \%$ yield).

White solid. ${ }^{1} \mathbf{H}$ NMR $\left(300 \mathrm{MHz}, \mathrm{CDCl}_{3}\right) \delta 8.71(\mathrm{~d}, J=15.7 \mathrm{~Hz}, 1 \mathrm{H}), 8.17(\mathrm{~d}, J=8.3 \mathrm{~Hz}, 1 \mathrm{H}), 8.03-7.79$ (m, 3H), 7.70-7.47 (m, 3H), 6.55-6.43 (m, 1H).

${ }^{19}$ F NMR $\left(282 \mathrm{MHz}, \mathrm{CDCl}_{3}\right) \delta 26.1(\mathrm{~d}, J=7.1 \mathrm{M} \mathrm{Hz}, 1 \mathrm{~F})$.

MS (EI, m/z): $200\left(\mathbf{M}^{+}\right)$.

The characterization data are consistent with the previous report. ${ }^{11}$

\section{(E)-3-(3-Chlorophenyl)acryloyl fluoride (3u)}<smiles>O=C(F)/C=C/c1cccc(Cl)c1</smiles>

Performed on $0.5 \mathrm{mmol}$ scale, eluted with petroleum ether/ethyl acetate $=20 / 1$ to give $3 \mathbf{u}(71.1 \mathrm{mg}, 77 \%$ yield).

Colorless oil. ${ }^{1} \mathbf{H}$ NMR $\left(400 \mathrm{MHz}, \mathrm{CDCl}_{3}\right) \delta 7.76(\mathrm{~d}, J=16.0 \mathrm{~Hz}, 1 \mathrm{H}), 7.54(\mathrm{~d}, J=2.1 \mathrm{~Hz}, 1 \mathrm{H}), 7.47-7.33$ (m, 3H), $6.37(\mathrm{dd}, J=16.0,7.0 \mathrm{~Hz}, 1 \mathrm{H})$.

${ }^{19}$ F NMR $\left(376 \mathrm{MHz}, \mathrm{CDCl}_{3}\right) \delta 25.9(\mathrm{~s}, 1 \mathrm{~F})$.

${ }^{13} \mathrm{C}$ NMR $\left(101 \mathrm{MHz}, \mathrm{CDCl}_{3}\right) \delta 156.6\left(\mathrm{~d},{ }^{1} J_{\mathrm{CF}}=339.2 \mathrm{~Hz}\right), 149.6\left(\mathrm{~d},{ }^{3} J_{\mathrm{CF}}=6.0 \mathrm{~Hz}\right), 135.2,134.8,131.7$, $130.5,128.4,126.9,113.6\left(\mathrm{~d},{ }^{2} J_{\mathrm{CF}}=67.9 \mathrm{~Hz}\right)$.

MS (EI, m/z): $184\left(\mathbf{M}^{+}\right)$.

HRMS (EI) m/z: [M] $]^{+}$Calcd. for $\mathrm{C}_{9} \mathrm{H}_{6} \mathrm{ClFO}$ : 184.0086; Found: 184.0088 .

IR (film): 3076, 1800, 1633, 1567, 1473, 1432, 1317, 1258, 1218, 1192, 1110, 982, 887, 855, 787, 668, 584, $433 \mathrm{~cm}^{-1}$.

\section{Detailed Procedure for Deoxyfluorination of carboxylic acid 1u}

To an oven-dried sealed tube charged with a magnetic stirrer bar, were successively added carboxylic acid 
1u (1 mmol, $0.1826 \mathrm{~g})$, CpFluor 2 (1.5 mmol, 1.6 equiv, $365 \mathrm{mg})$, and $\mathrm{CH}_{2} \mathrm{Cl}_{2}(5 \mathrm{~mL})$. The reaction mixture was immersed into $50{ }^{\circ} \mathrm{C}$ oil bath and stirred for $4 \mathrm{~h}$. After the reaction was completed, the reaction mixture was cooled to room temperature and extracted with $\mathrm{H}_{2} \mathrm{O}$ and $\mathrm{CH}_{2} \mathrm{Cl}_{2}$. The obtained organic layer was dried over $\mathrm{MgSO}_{4}$, then concentrated under vacuum. The residue was purified by flash chromatography with petroleum ether/ethyl acetate as eluent to afford the corresponding acyl fluorides $\mathbf{3 u}$ (137.9 mg from 1 mmol of substrate, $75 \%$ yield, colorless oil).

\section{4-(N,N-dipropylsulfamoyl)benzoyl fluoride (3v)}<smiles>CCCN(CCC)S(=O)(=O)c1ccc(C(=O)F)cc1</smiles>

Performed on $0.5 \mathrm{mmol}$ scale, eluted with petroleum ether/ethyl acetate $=3 / 1$ to give $\mathbf{3 v}(117.8 \mathrm{mg}, 82 \%$ yield).

White solid. ${ }^{1} \mathbf{H}$ NMR $\left(400 \mathrm{MHz}, \mathrm{CDCl}_{3}\right) \delta 8.14(\mathrm{~d}, J=8.5 \mathrm{~Hz}, 2 \mathrm{H}), 7.93(\mathrm{~d}, J=7.8 \mathrm{~Hz}, 2 \mathrm{H}), 3.12-3.06(\mathrm{~m}$, $4 \mathrm{H}), 1.57-1.47(\mathrm{~m}, 4 \mathrm{H}), 0.83(\mathrm{t}, J=7.4 \mathrm{~Hz}, 6 \mathrm{H})$.

${ }^{19}$ F NMR $\left(376 \mathrm{MHz}, \mathrm{CDCl}_{3}\right) \delta 20.1(\mathrm{~s}, 1 \mathrm{~F})$.

$\operatorname{MS}(\mathbf{E I}, \mathrm{m} / \mathrm{z}): 287\left(\mathbf{M}^{+}\right)$.

The characterization data are consistent with the previous report. ${ }^{3}$

\section{4-(1-(3,5,5,8,8-Pentamethyl-5,6,7,8-tetrahydronaphthalen-2-yl)vinyl)benzoyl fluoride} (3w)<smiles>C=C(c1ccc(C(=O)F)cc1)c1cc2c(cc1C)C(C)(C)CCC2(C)C</smiles>

Performed on $0.5 \mathrm{mmol}$ scale, eluted with petroleum ether/ethyl acetate $=3 / 1$ to give $\mathbf{3 w}(66.6 \mathrm{mg}, 38 \%$ yield). 
White solid. ${ }^{1} \mathbf{H}$ NMR $\left(400 \mathrm{MHz}, \mathrm{CDCl}_{3}\right) \delta 8.03(\mathrm{~d}, J=8.4 \mathrm{~Hz}, 2 \mathrm{H}), 7.37(\mathrm{~d}, J=8.4 \mathrm{~Hz}, 2 \mathrm{H}), 7.13(\mathrm{~s}, 1 \mathrm{H})$, $7.08(\mathrm{~s}, 1 \mathrm{H}), 5.83(\mathrm{~d}, J=1.4 \mathrm{~Hz}, 1 \mathrm{H}), 5.34(\mathrm{~d}, J=1.3 \mathrm{~Hz}, 1 \mathrm{H}), 1.94$ (s, 3H), 1.69 (s, 4H), 1.29 (d, $J=10.9$ $\mathrm{Hz}, 12 \mathrm{H})$.

${ }^{19}$ F NMR $\left(376 \mathrm{MHz}, \mathrm{CDCl}_{3}\right) \delta 17.9(\mathrm{~s}, 1 \mathrm{~F})$.

MS (EI, m/z): $350\left(\mathrm{M}^{+}\right)$.

The characterization data are consistent with the previous report. ${ }^{9}$ 


\section{Procedures for One-Pot Deoxyfluorination/Amination of Carboxylic Acids}

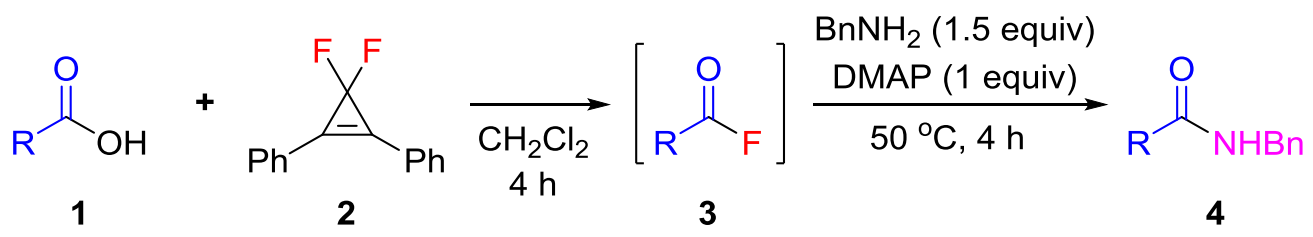

To an oven-dried sealed tube charged with a magnetic stirrer bar, were successively added carboxylic acid $\mathbf{1}$ (0.5 mmol), CpFluor 2 (0.8 mmol, 1.6 equiv, $183 \mathrm{mg})$, and $\mathrm{CH}_{2} \mathrm{Cl}_{2}(2.5 \mathrm{~mL})$ for $4 \mathrm{~h}$. Then benzylamine ( $0.75 \mathrm{mmol}, 1.5$ equiv, $81.1 \mathrm{mg}$ ) and 4-dmethylaminopyridine ( $0.5 \mathrm{mmol}, 1$ equiv, $122.2 \mathrm{mg})$ were added to the reaction mixture. The reaction mixture was immersed into $50{ }^{\circ} \mathrm{C}$ oil bath and stirred for $4 \mathrm{~h}$. After cooling to room temperature, to the mixture was added saturated aqueous $\mathrm{NaHCO}_{3}$ (ca. $5 \mathrm{~mL}$ ) and extracted with EtOAc (ca. $5 \mathrm{~mL} \times 3$ ). The combined organic layer was dried over $\mathrm{MgSO}_{4}$, and then filtration, the filtrate was concentrated under reduced pressure. The residue was purified by column chromatography on silica gel with petroleum ether/ethyl acetate as eluent to afford the corresponding final product 4 .

\section{$N$-Benzyl-2-(naphthalen-2-yl)acetamide (4x)}<smiles>O=C(Cc1ccc2ccccc2c1)Nc1ccccc1</smiles>

Performed on $0.5 \mathrm{mmol}$ scale, eluted with petroleum ether/ethyl acetate $=5 / 1$ to give $4 \mathbf{x}(75.7 \mathrm{mg}, 55 \%$ yield).

Pale yellow solid. ${ }^{1}$ H NMR $\left(400 \mathrm{MHz}, \mathrm{CDCl}_{3}\right) \delta 7.80(\mathrm{dd}, J=16.1,7.1 \mathrm{~Hz}, 3 \mathrm{H}), 7.70(\mathrm{~s}, 1 \mathrm{H}), 7.47(\mathrm{td}, J=$ 6.7, 6.1, 3.3 Hz, 2H), $7.37(\mathrm{dd}, J=8.5,1.8 \mathrm{~Hz}, 1 \mathrm{H}), 7.28-7.20(\mathrm{~m}, 3 \mathrm{H}), 7.20-7.11(\mathrm{~m}, 2 \mathrm{H}), 5.83(\mathrm{~s}, 1 \mathrm{H})$, $4.39(\mathrm{~d}, J=5.8 \mathrm{~Hz}, 2 \mathrm{H}), 3.76(\mathrm{~s}, 2 \mathrm{H})$.

MS (EI, m/z): $275\left(\mathbf{M}^{+}\right)$.

The characterization data are consistent with the previous report. ${ }^{12}$

\section{$N$-Benzyl-2-(4-methoxyphenyl)acetamide (4y)}<smiles>COc1ccc(CC(=O)Nc2ccccc2)cc1</smiles> 
Performed on $0.5 \mathrm{mmol}$ scale, eluted with petroleum ether/ethyl acetate $=5 / 1$ to give $4 \mathbf{y}(88.1 \mathrm{mg}, 69 \%$ yield).

White solid. ${ }^{1} \mathbf{H}$ NMR $\left(400 \mathrm{MHz}, \mathrm{CDCl}_{3}\right) \delta$ 7.30-7.22 (m, 3H), 7.21-7.11 (m, 4H), $6.85(\mathrm{~d}, J=8.6 \mathrm{~Hz}, 2 \mathrm{H})$, $5.76(\mathrm{~s}, 1 \mathrm{H}), 4.38(\mathrm{~d}, J=5.8 \mathrm{~Hz}, 2 \mathrm{H}), 3.77(\mathrm{~s}, 3 \mathrm{H}), 3.54(\mathrm{~s}, 2 \mathrm{H})$.

MS (EI, m/z): $255\left(\mathrm{M}^{+}\right)$.

The characterization data are consistent with the previous report. ${ }^{12}$

$N$-Benzyl-4-phenylbutanamide (4z)<smiles>O=C(CCCc1ccccc1)Nc1ccccc1</smiles>

Performed on $0.5 \mathrm{mmol}$ scale, eluted with petroleum ether/ethyl acetate $=5 / 1$ to give $\mathbf{4 z}(105.1 \mathrm{mg}, 83 \%$ yield).

White solid. ${ }^{1} \mathbf{H}$ NMR $\left(400 \mathrm{MHz}, \mathrm{CDCl}_{3}\right) \delta$ 7.37-7.21 (m, 7H), 7.21-7.09 (m, 3H), $5.93(\mathrm{~s}, 1 \mathrm{H}), 4.39(\mathrm{~d}, J=$ $5.7 \mathrm{~Hz}, 2 \mathrm{H}), 2.64(\mathrm{t}, J=7.5 \mathrm{~Hz}, 2 \mathrm{H}), 2.19(\mathrm{t}, J=7.5 \mathrm{~Hz}, 2 \mathrm{H}), 2.02-1.93(\mathrm{~m}, 2 \mathrm{H})$.

MS (EI, m/z): $253\left(\mathrm{M}^{+}\right)$.

The characterization data are consistent with the previous report. ${ }^{13}$

\section{$N$-Benzyl-3-phenylpropiolamide (4aa)}<smiles>O=C(C#Cc1ccccc1)Nc1ccccc1</smiles>

Performed on $0.5 \mathrm{mmol}$ scale, eluted with petroleum ether/ethyl acetate $=5 / 1$ to give $\mathbf{3 a}(77 \mathrm{mg}, 65 \%$ yield).

White solid. Major rotamer: ${ }^{1} \mathrm{H}$ NMR $\left(400 \mathrm{MHz}, \mathrm{CDCl}_{3}\right) \delta 7.50(\mathrm{~d}, J=7.1 \mathrm{~Hz}, 2 \mathrm{H})$, 7.43-7.26 $(\mathrm{m}, 8 \mathrm{H})$, $6.58(\mathrm{~s}, 1 \mathrm{H}), 4.51(\mathrm{~d}, J=5.9 \mathrm{~Hz}, 2 \mathrm{H})$. White solid. Minor rotamer: ${ }^{1} \mathbf{H} \mathbf{N M R}\left(400 \mathrm{MHz}, \mathrm{CDCl}_{3}\right) \delta 7.50(\mathrm{~d}$, $J=7.1 \mathrm{~Hz}, 2 \mathrm{H}), 7.43-7.26(\mathrm{~m}, 8 \mathrm{H}), 6.31(\mathrm{~s}, 1 \mathrm{H}), 4.66(\mathrm{~d}, J=6.5 \mathrm{~Hz}, 1 \mathrm{H})$.

MS (EI, m/z): $235\left(\mathbf{M}^{+}\right)$.

The characterization data are consistent with the previous report. ${ }^{14}$ 


\section{$N$-Benzyl-6-bromonicotinamide (4ab)}<smiles>O=C(Nc1ccccc1)c1ccc(Br)nc1</smiles>

Performed on $0.5 \mathrm{mmol}$ scale, eluted with petroleum ether/ethyl acetate $=5 / 1$ to give $4 \mathbf{a b}(106.8 \mathrm{mg}, 73 \%$ yield).

Yellow solid. m.p. 136-137 ${ }^{\circ} \mathrm{C} .{ }^{1} \mathbf{H}$ NMR $\left(400 \mathrm{MHz}, \mathrm{CDCl}_{3}\right) \delta 8.64(\mathrm{~d}, J=2.5 \mathrm{~Hz}, 1 \mathrm{H}), 7.90(\mathrm{dd}, J=8.2$, $2.5 \mathrm{~Hz}, 1 \mathrm{H}), 7.47(\mathrm{~d}, J=8.3 \mathrm{~Hz}, 1 \mathrm{H}), 7.27(\mathrm{q}, J=6.6 \mathrm{~Hz}, 5 \mathrm{H}), 6.98(\mathrm{t}, J=5.8 \mathrm{~Hz}, 1 \mathrm{H}), 4.54(\mathrm{~d}, J=5.7 \mathrm{~Hz}$, $2 \mathrm{H})$.

${ }^{13}$ C NMR (101 MHz, $\left.\mathrm{CDCl}_{3}\right) \delta 164.6,148.4,145.2,137.6,137.5,129.3,128.8,128.2,127.9,127.8,44.2$.

MS (EI, m/z): $290\left(\mathbf{M}^{+}\right)$.

HRMS (EI) m/z: [M] $]^{+}$Calcd. for $\mathrm{C}_{13} \mathrm{H}_{11} \mathrm{BrN}_{2} \mathrm{O}$ : 290.0049; Found: 290.0059.

IR (film): 3284, 3064, 3034, 1640, 1580, 1540, 1494, 1453, 1321, 1090, 1048, 1022, 976, 848, 769, 694, $630 \mathrm{~cm}^{-1}$.

\section{$N$-Benzyl-4-oxo-4H-chromene-2-carboxamide (4ac)}<smiles>O=C(Nc1ccccc1)c1cc(=O)c2ccccc2o1</smiles>

Performed on $0.5 \mathrm{mmol}$ scale, eluted with petroleum ether/ethyl acetate $=5 / 1$ to give $\mathbf{4 a c}(57.9 \mathrm{mg}, 41 \%$ yield).

White solid. ${ }^{1} \mathbf{H}$ NMR $\left(400 \mathrm{MHz}, \mathrm{CDCl}_{3}\right) \delta 8.26(\mathrm{dd}, J=8.0,1.7 \mathrm{~Hz}, 1 \mathrm{H}), 7.78(\mathrm{td}, J=8.7,7.1,1.7 \mathrm{~Hz}$, 1H), $7.60-7.36(\mathrm{~m}, 8 \mathrm{H}), 7.26(\mathrm{~s}, 1 \mathrm{H}), 4.75(\mathrm{~d}, J=5.9 \mathrm{~Hz}, 2 \mathrm{H})$.

MS (EI, m/z): $280\left(\mathrm{M}^{+}\right)$.

The characterization data are consistent with the previous report. ${ }^{15}$

\section{$N$-Benzyl-2-((1-benzyl-1H-indazol-3-yl)oxy)acetamide (4ad)}




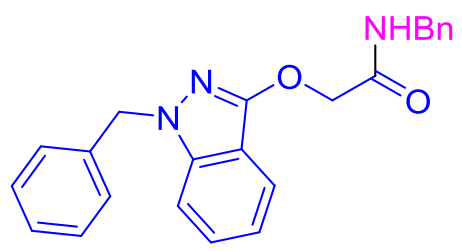

Performed on $0.5 \mathrm{mmol}$ scale, eluted with petroleum ether/ethyl acetate $=3 / 1$ to give $\mathbf{4 a d}(144.4 \mathrm{mg}, 78 \%$ yield).

Yellow oil. ${ }^{1} \mathbf{H}$ NMR (400 MHz, $\left.\mathrm{CDCl}_{3}\right) \delta 7.66(\mathrm{dd}, J=8.1,1.0 \mathrm{~Hz}, 1 \mathrm{H}), 7.38-7.16$ (m, 12H), 7.09-6.99 (m, 2H), $5.37(\mathrm{~s}, 2 \mathrm{H}), 4.98(\mathrm{~s}, 2 \mathrm{H}), 4.55(\mathrm{~d}, J=6.0 \mathrm{~Hz}, 2 \mathrm{H})$.

${ }^{13}$ C NMR (101 MHz, $\left.\mathrm{CDCl}_{3}\right) \delta 168.3,154.3,141.6,137.9,137.0,128.7,128.6,127.7,127.7,127.6,127.5$, $127.2,119.7,119.6,112.3,109.2,68.2,52.5,42.9$.

MS (EI, m/z): $372\left(\mathbf{M}^{+}\right)$.

HRMS (ESI) m/z: [M+H] $]^{+}$Calcd. for $\mathrm{C}_{23} \mathrm{H}_{22} \mathrm{~N}_{3} \mathrm{O}_{2}: 372.1707$; Found: 372.1704 .

IR (film): 3434, 3323, 3064, 1675, 1619, 1581, 1521, 1496, 1454, 1432, 1340, 1186, 909, 770, 733, 700, $649 \mathrm{~cm}^{-1}$.

\section{(Z)- $N$-Benzyl-2-(5-fluoro-2-methyl-1-(4-(methylsulfinyl)benzylidene)-1H-inden-3-yl)acet} amide (4ae)<smiles>CC1=C(CC(=O)NCc2ccccc2)c2cc(F)ccc2/C1=C\c1ccc(S(C)=O)cc1</smiles>

Performed on $0.5 \mathrm{mmol}$ scale, eluted with petroleum ether/ethyl acetate $=3 / 1$ to give 4 ae $(170.7 \mathrm{mg}, 75 \%$ yield).

Yellow solid. ${ }^{1} \mathbf{H}$ NMR $\left(400 \mathrm{MHz}, \mathrm{CDCl}_{3}\right) \delta$ 7.68-7.52 (m, 4H), 7.25-7.06 (m, 7H), 6.96-6.86 (m, 2H), $6.51(\mathrm{td}, J=8.8,2.4 \mathrm{~Hz}, 1 \mathrm{H}), 4.34(\mathrm{~d}, J=5.7 \mathrm{~Hz}, 2 \mathrm{H}), 3.48(\mathrm{~s}, 2 \mathrm{H}), 2.71(\mathrm{~s}, 3 \mathrm{H}), 2.16(\mathrm{~s}, 3 \mathrm{H})$.

${ }^{19}$ F NMR $\left(376 \mathrm{MHz}, \mathrm{CDCl}_{3}\right) \delta-112.60(\mathrm{~m}, 1 \mathrm{~F})$.

MS (EI, m/z): $446\left(\mathrm{M}^{+}\right)$.

The characterization data are consistent with the previous report. ${ }^{16}$ 


\section{Procedures for Two-Step Acyl Fluorides Synthesis via in-situ Formed CpFluor}

5.1 Two-step [2+1] Cycloaddition/Deoxyfluorination for Aryl Acyl Fluorides Synthesis
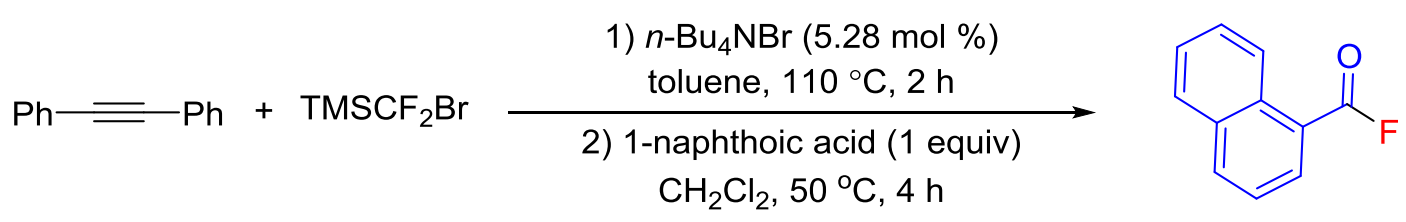

$3 c, 61 \%$

To an oven-dried sealed tube charged with a magnetic stirrer bar, were successively added 1,2-diphenylethyne (0.88 mmol, 1.76 equiv, $156.8 \mathrm{mg}),{ }^{n} \mathrm{Bu}_{4} \mathrm{NBr}(0.0264 \mathrm{mmol}, 5.28 \mathrm{~mol} \%, 8.5 \mathrm{mg})$, toluene $(2 \mathrm{~mL})$ and $\mathrm{TMSCF}_{2} \mathrm{Br}(1.32 \mathrm{mmol}, 2.64$ equiv, $268.1 \mathrm{mg})$. The reaction mixture was immersed into $110{ }^{\circ} \mathrm{C}$ oil bath and stirred for 2 hours. After the reaction mixture was cooled to room temperature, the solvent toluene was removed by pump. Then directly added 1-naphthoic acid $1 \mathbf{c}(0.5 \mathrm{mmol}, 1$ equiv, 86.1 $\mathrm{mg})$ and $\mathrm{CH}_{2} \mathrm{Cl}_{2}(2.5 \mathrm{~mL})$. The reaction mixture was immersed again into $50{ }^{\circ} \mathrm{C}$ oil bath and stirred for 4 hours. After the reaction was completed, the reaction mixture was cooled to room temperature and extracted with $\mathrm{H}_{2} \mathrm{O}$ and $\mathrm{CH}_{2} \mathrm{Cl}_{2}$. The combined organic layer was dried over $\mathrm{MgSO}_{4}$, then concentrated under vacuum. The crude product was purified by flash chromatography with petroleum ether/ethyl acetate as eluent to afford the corresponding acyl fluorides $3 \mathbf{c}$ in $61 \%$ yield.

\subsection{Two-Step [2+1] Cycloaddition/Deoxyfluorination for Alkyl Acyl Fluorides Synthesis}

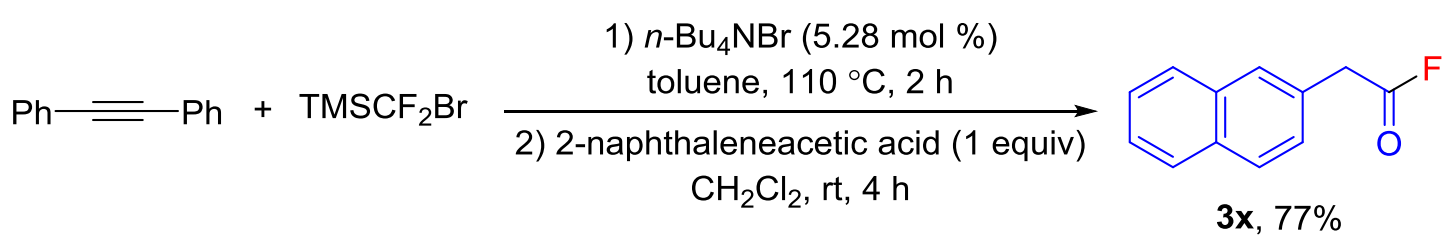

To an oven-dried sealed tube charged with a magnetic stirrer bar, were successively added 1,2-diphenylethyne (0.88 mmol, 1.76 equiv, $156.8 \mathrm{mg}),{ }^{n} \mathrm{Bu}_{4} \mathrm{NBr}(0.0264 \mathrm{mmol}, 5.28 \mathrm{~mol} \%, 8.5 \mathrm{mg})$, toluene $(2 \mathrm{~mL})$ and $\mathrm{TMSCF}_{2} \mathrm{Br}(1.32 \mathrm{mmol}, 2.64$ equiv, $268.1 \mathrm{mg})$. The reaction mixture was immersed into $110{ }^{\circ} \mathrm{C}$ oil bath and stirred for 2 hours. After the reaction mixture was cooled to room temperature, the solvent toluene was removed by pump. Then directly added 2-naphthaleneacetic acid $\mathbf{1 x}(0.5 \mathrm{mmol}, 1$ equiv, $93.1 \mathrm{mg})$, and $\mathrm{CH}_{2} \mathrm{Cl}_{2}(2.5 \mathrm{~mL})$. The reaction mixture was immersed again into $50{ }^{\circ} \mathrm{C}$ oil bath and stirred for 4 hours. After the reaction was completed, the reaction mixture was cooled to room temperature and quenched with $\mathrm{H}_{2} \mathrm{O}$ and $\mathrm{CH}_{2} \mathrm{Cl}_{2}$. The combined organic layer was dried over $\mathrm{MgSO}_{4}$, then concentrated 
under vacuum. Due to the instability of $\mathbf{3 x}$ on silica gel, the ${ }^{19} \mathrm{~F}$ NMR yield of $\mathbf{3 x}$ (77\%) was measured using benzotrifluoride as an internal standard. 


\section{Copies of NMR Charts for Products}
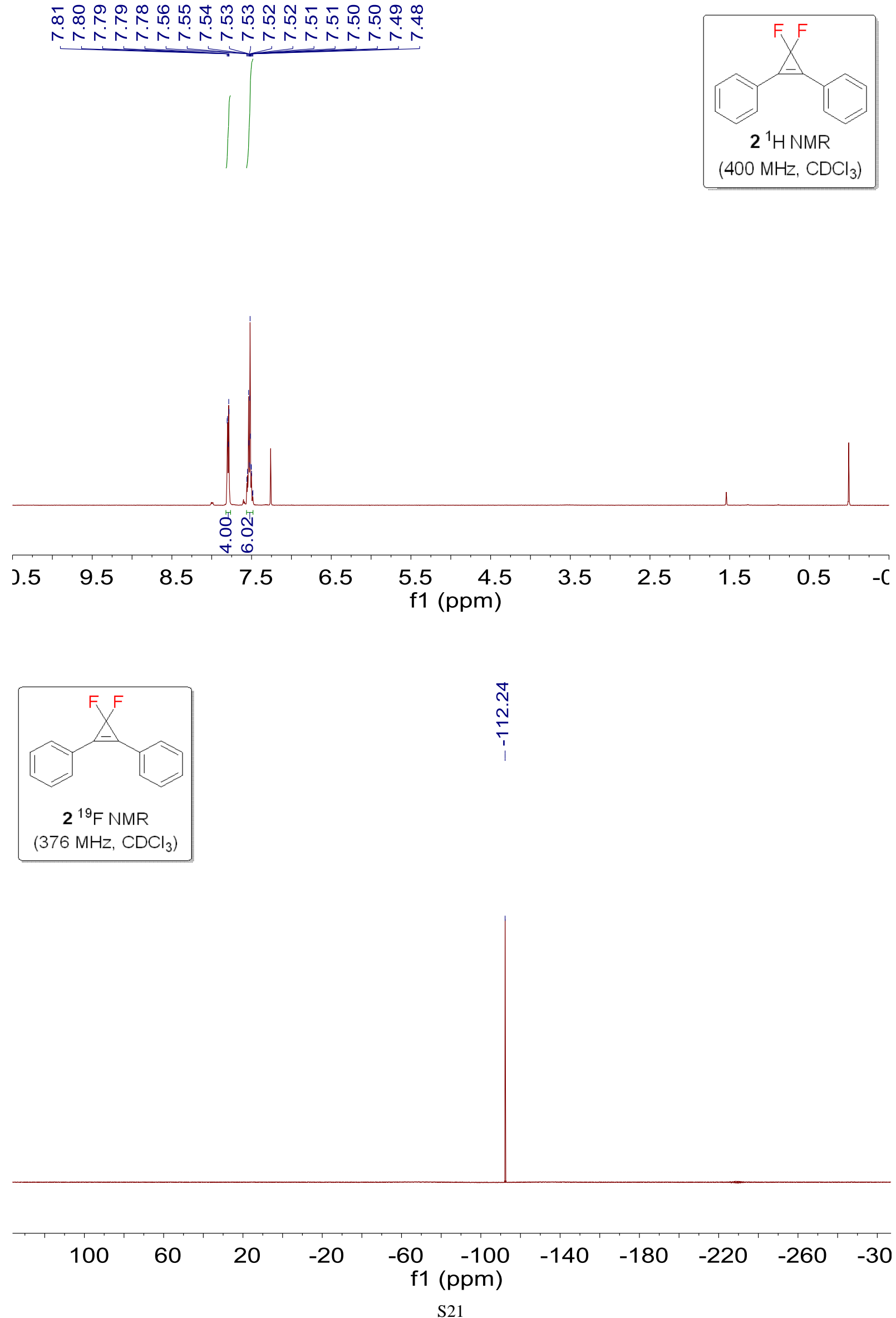


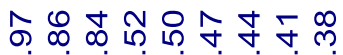

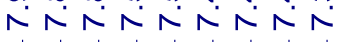
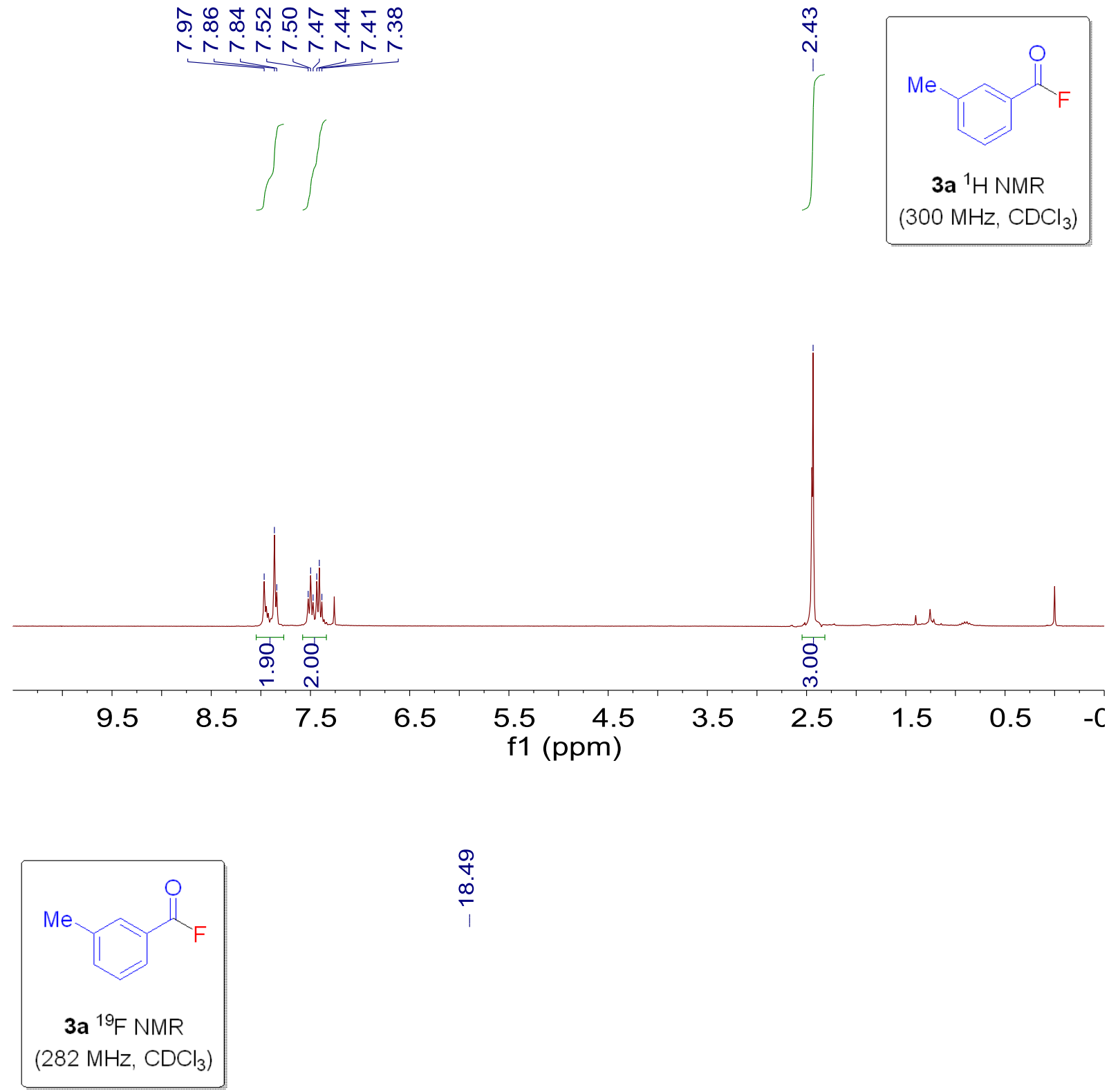

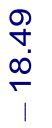

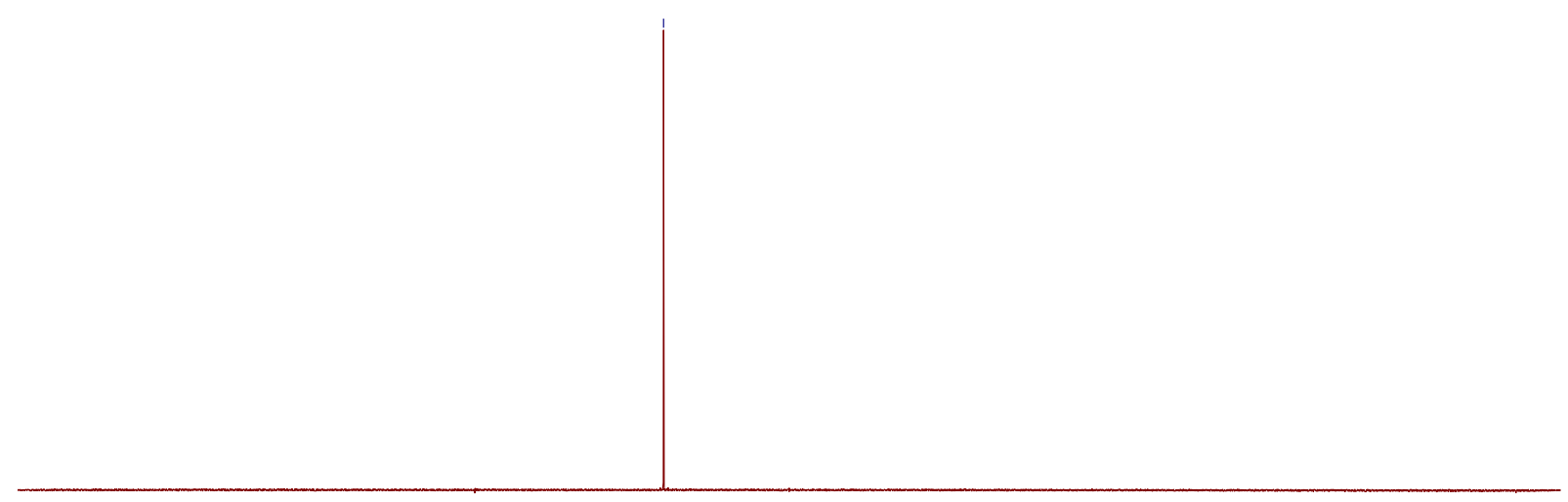

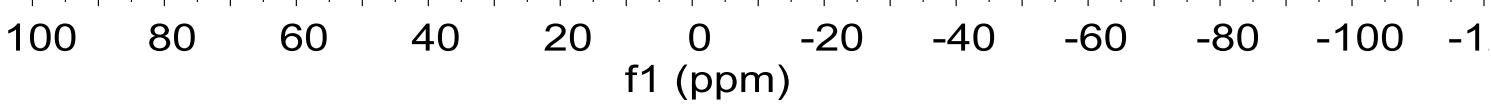




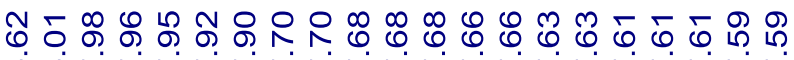

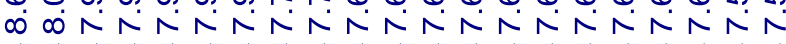
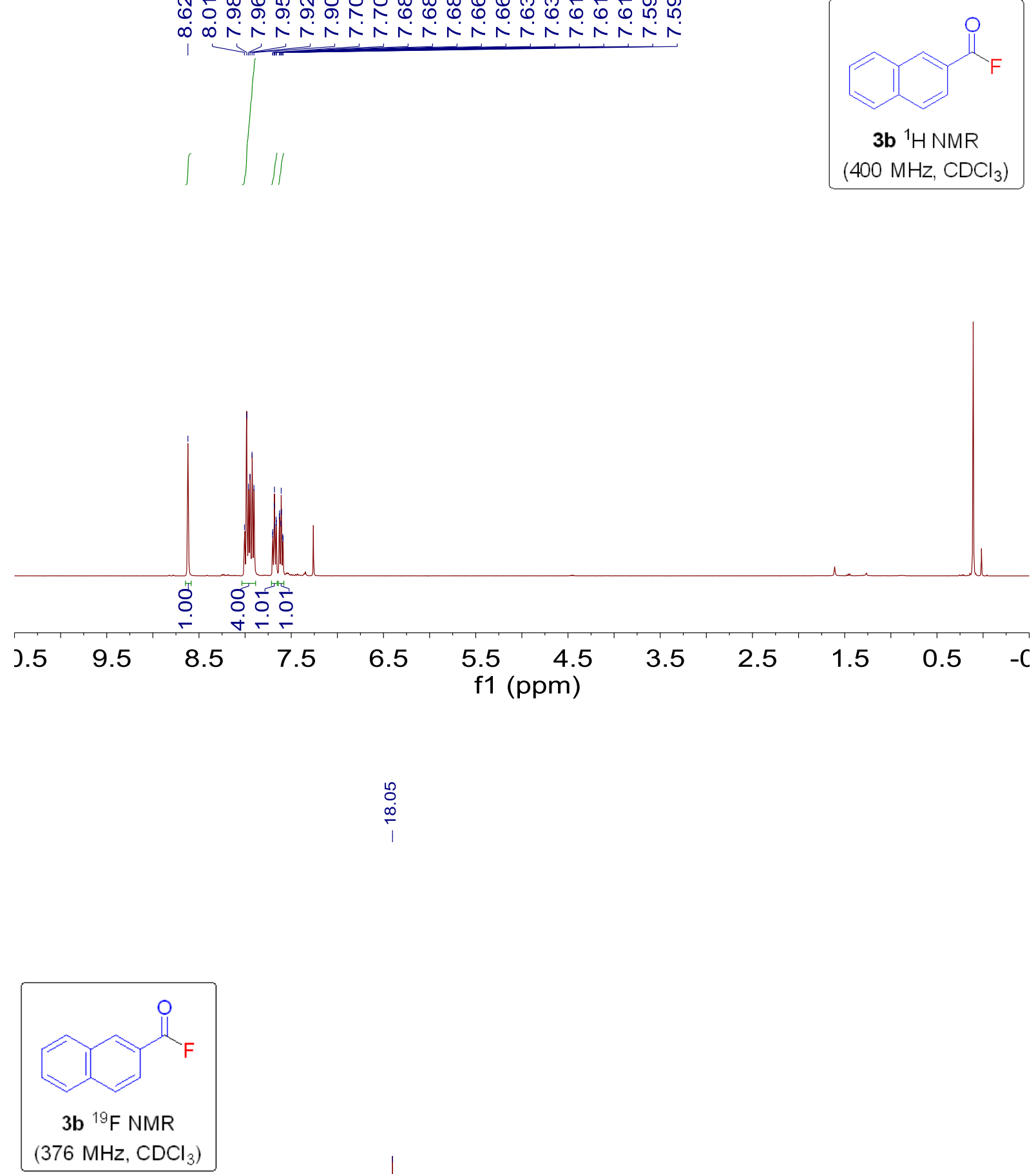

$\infty$
$\infty$
$\infty$
1

$\begin{array}{lllllllllllllllllllllll}140 & 120 & 100 & 80 & 60 & 40 & 20 & 0 & \begin{array}{c}-20 \\ \mathrm{f} 1(\mathrm{ppm})\end{array} & -40 & -60 & -80 & -100 & -120 & -140 & -160 & -180 & -21\end{array}$ 

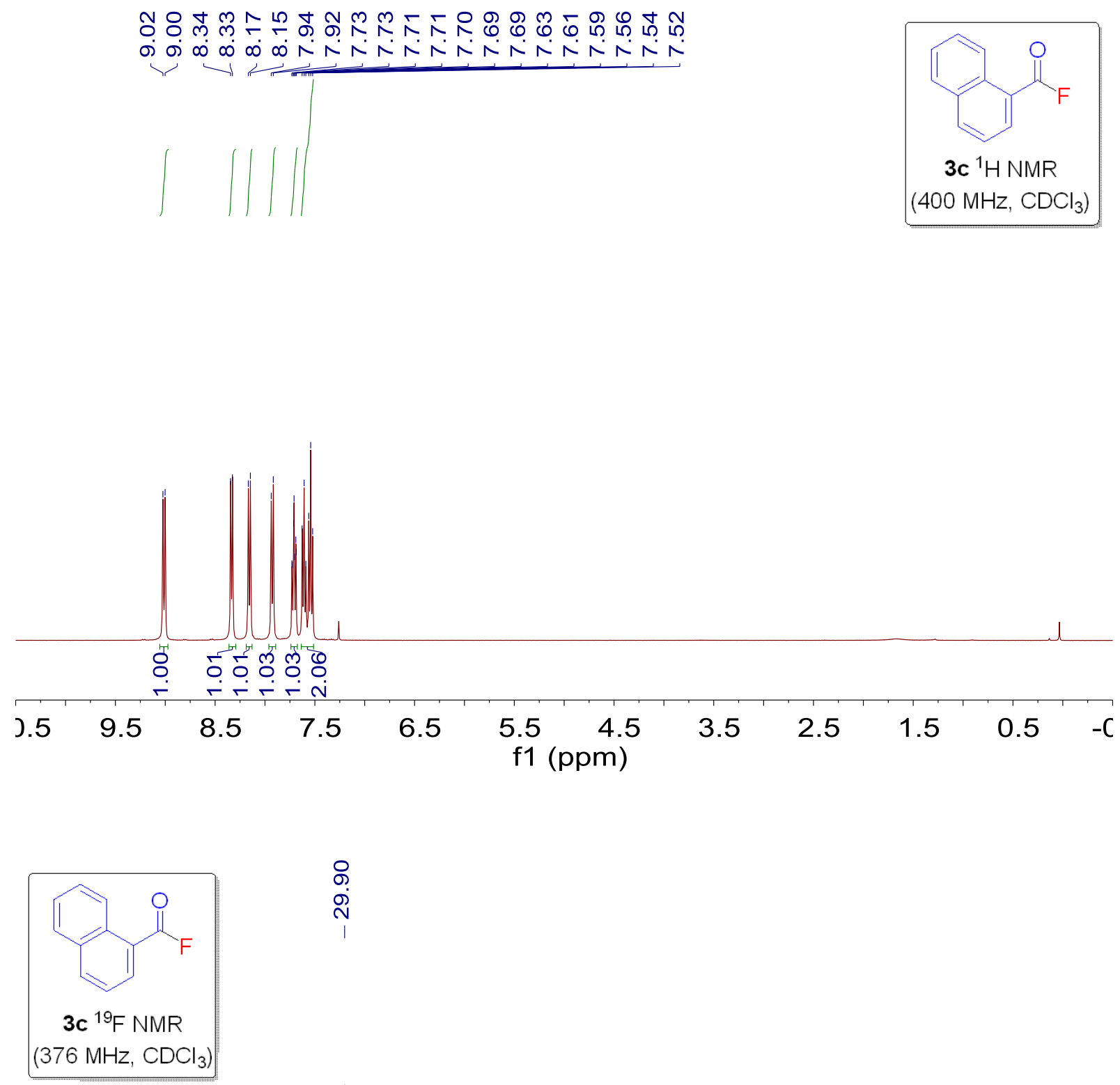

\section{:}

$\begin{array}{llllll}100 & 70 & 40 & 10 & -20 & -50\end{array}$

f1 (ppm)

$\begin{array}{lllll}-80 & -110 & -140 & -170 & -2(\end{array}$ 

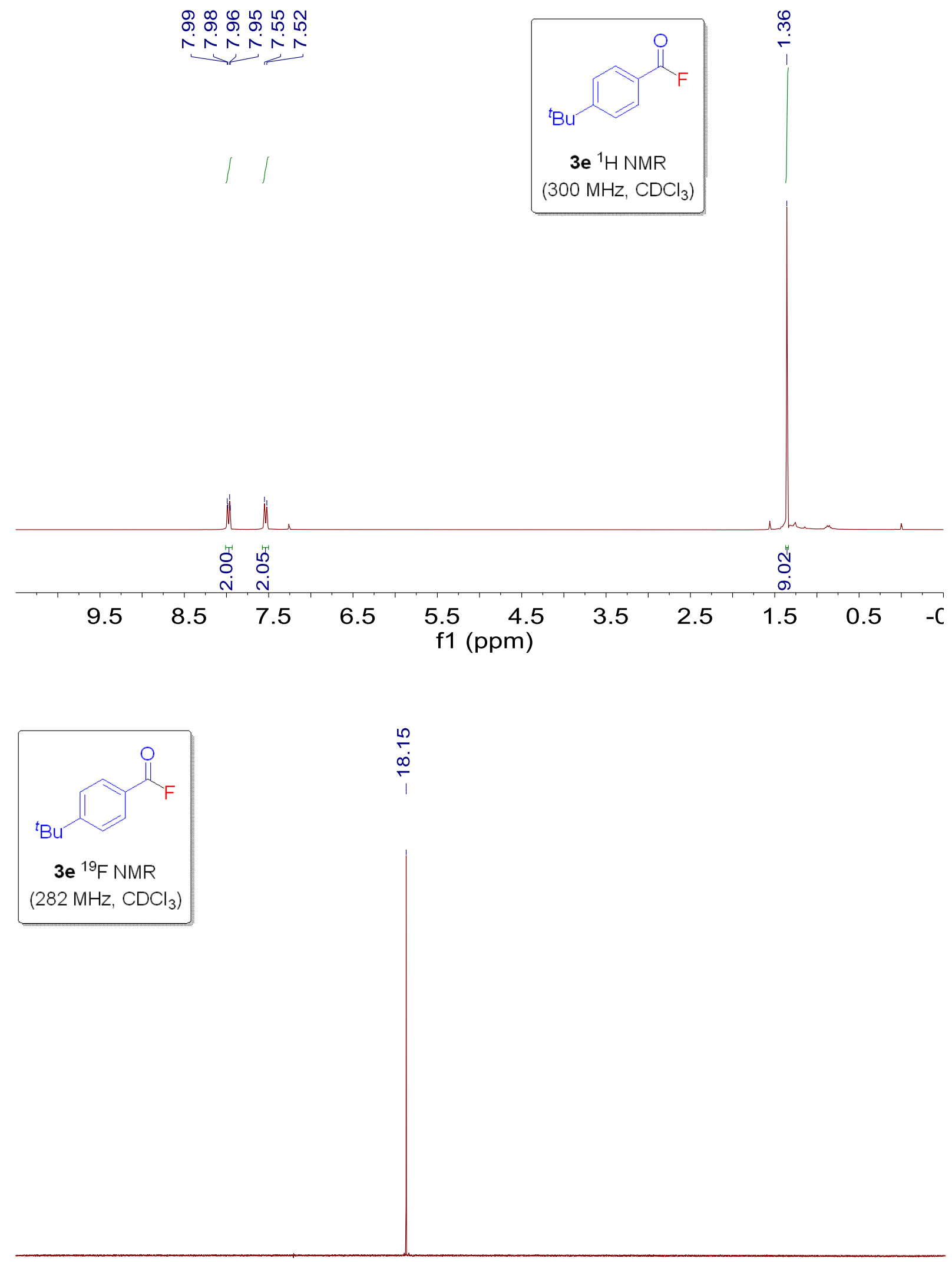

\begin{tabular}{|c|c|c|c|c|c|c|c|c|c|c|}
\hline 100 & 80 & 60 & 40 & 20 & $\begin{array}{c}0 \\
\text { f1 }(p p m\end{array}$ & -20 & -40 & -60 & -80 & -100 \\
\hline
\end{tabular}




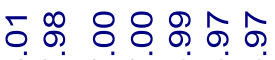

$\infty$
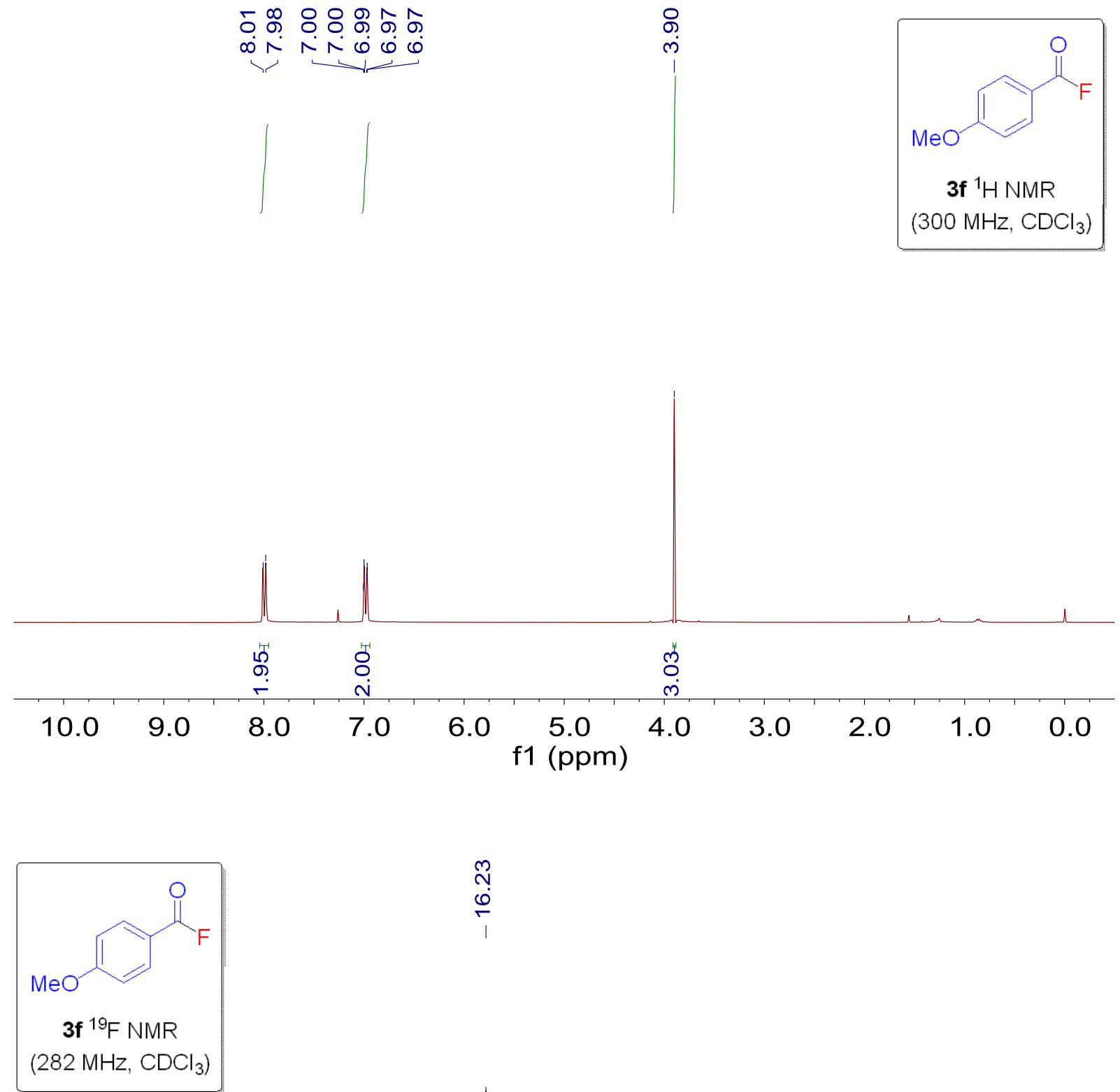

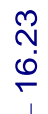

\begin{tabular}{|c|c|c|c|c|c|c|c|c|c|c|}
\hline 100 & 80 & 60 & 40 & 20 & $\begin{array}{c}0 \\
1 \text { (ppm }\end{array}$ & )$^{-20}$ & -40 & -60 & -80 & $\begin{array}{ll}-100 & -1\end{array}$ \\
\hline
\end{tabular}



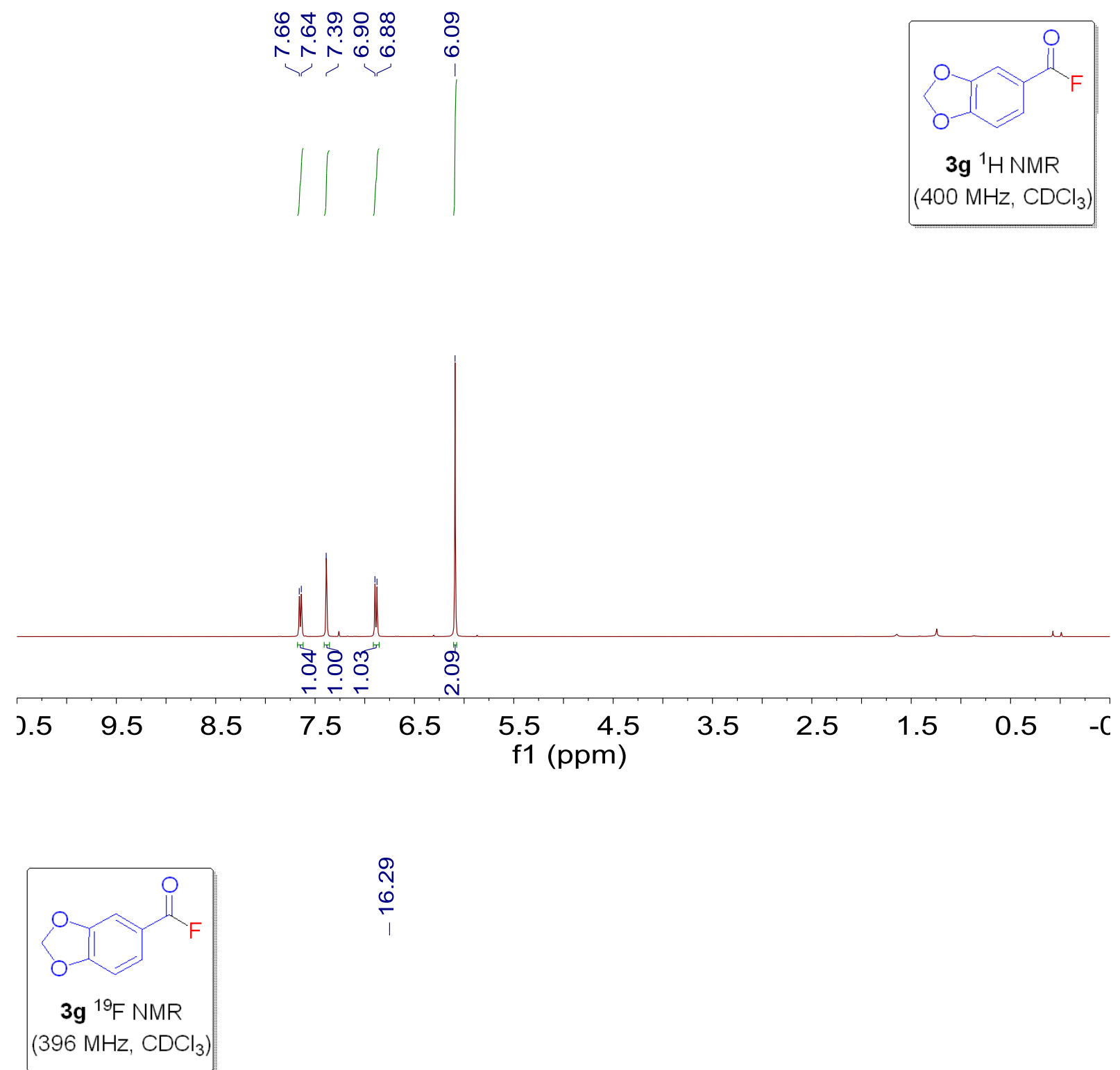

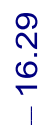

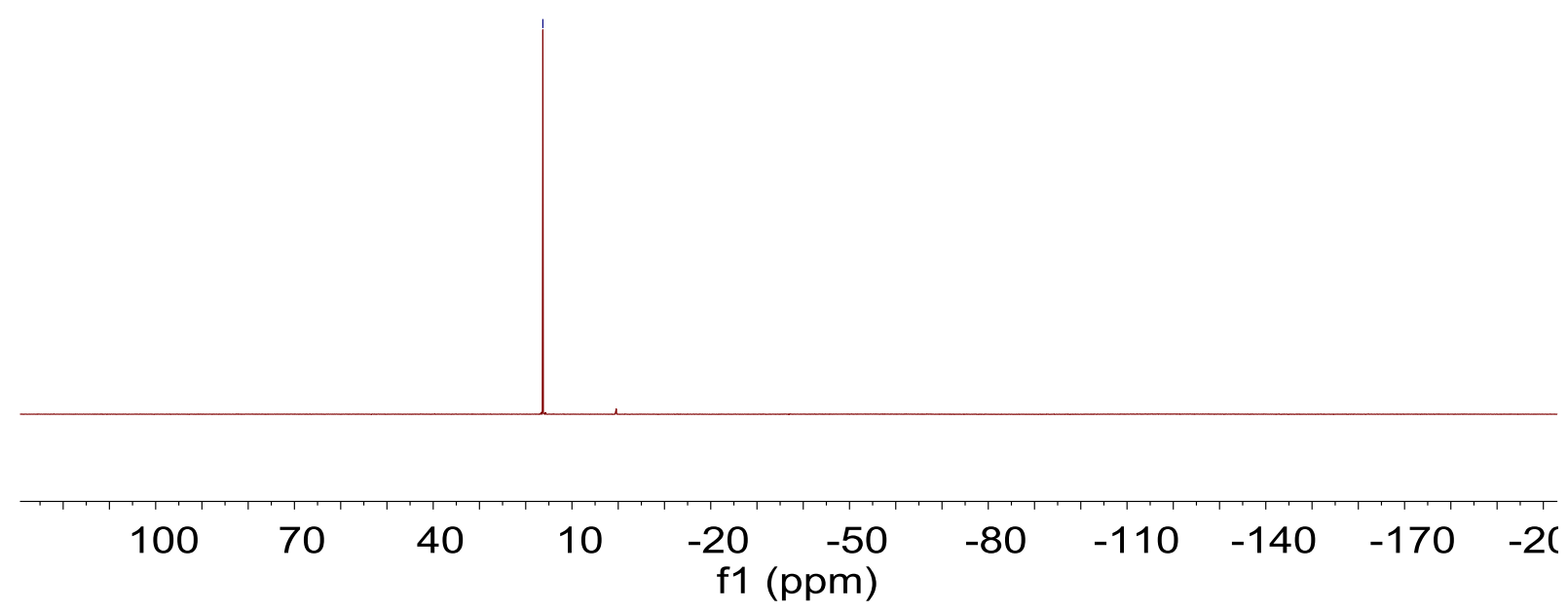


응ํํำ

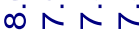
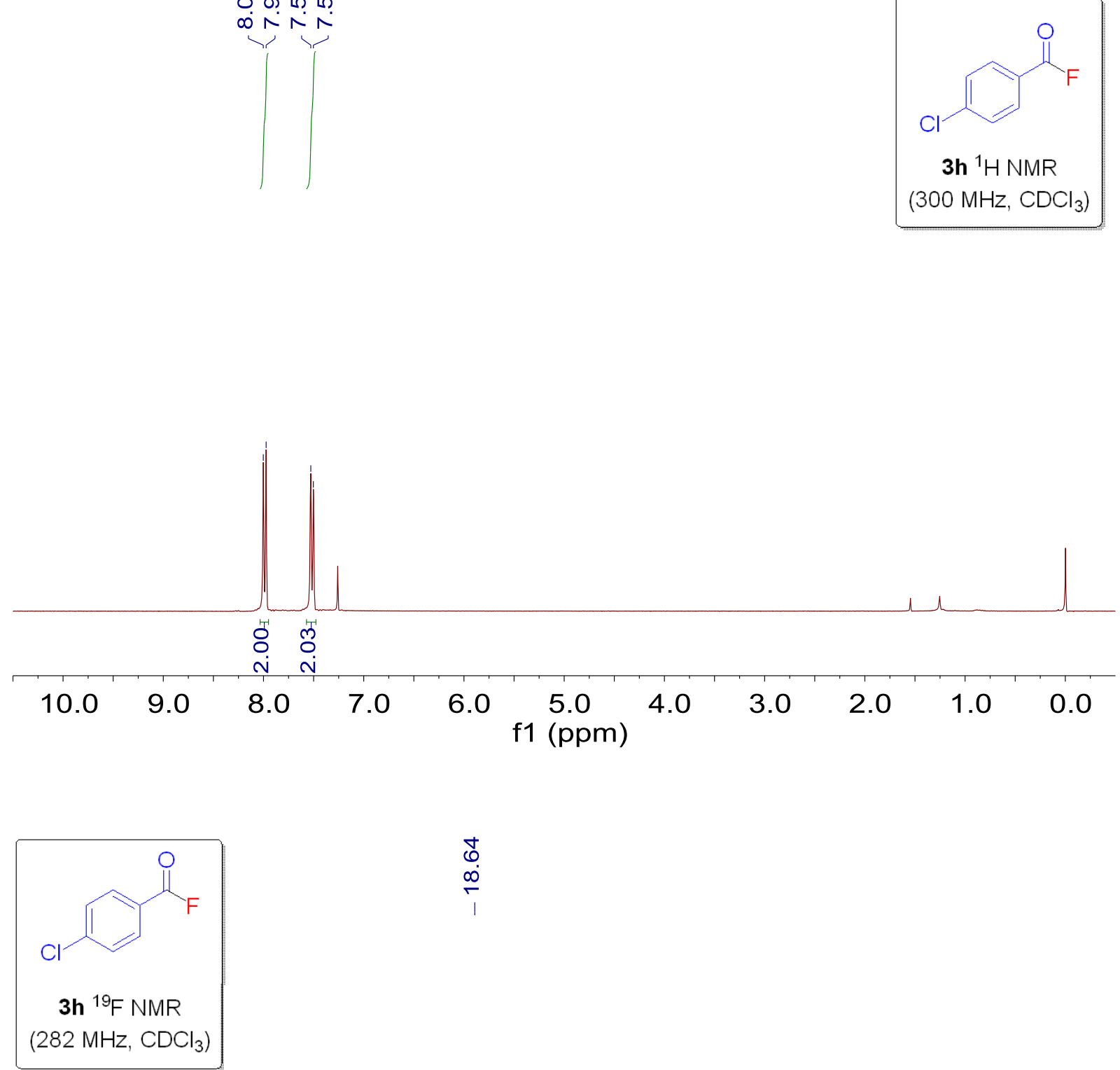

\begin{tabular}{l}
$\stackrel{+}{0}$ \\
$\infty$ \\
\hline
\end{tabular}

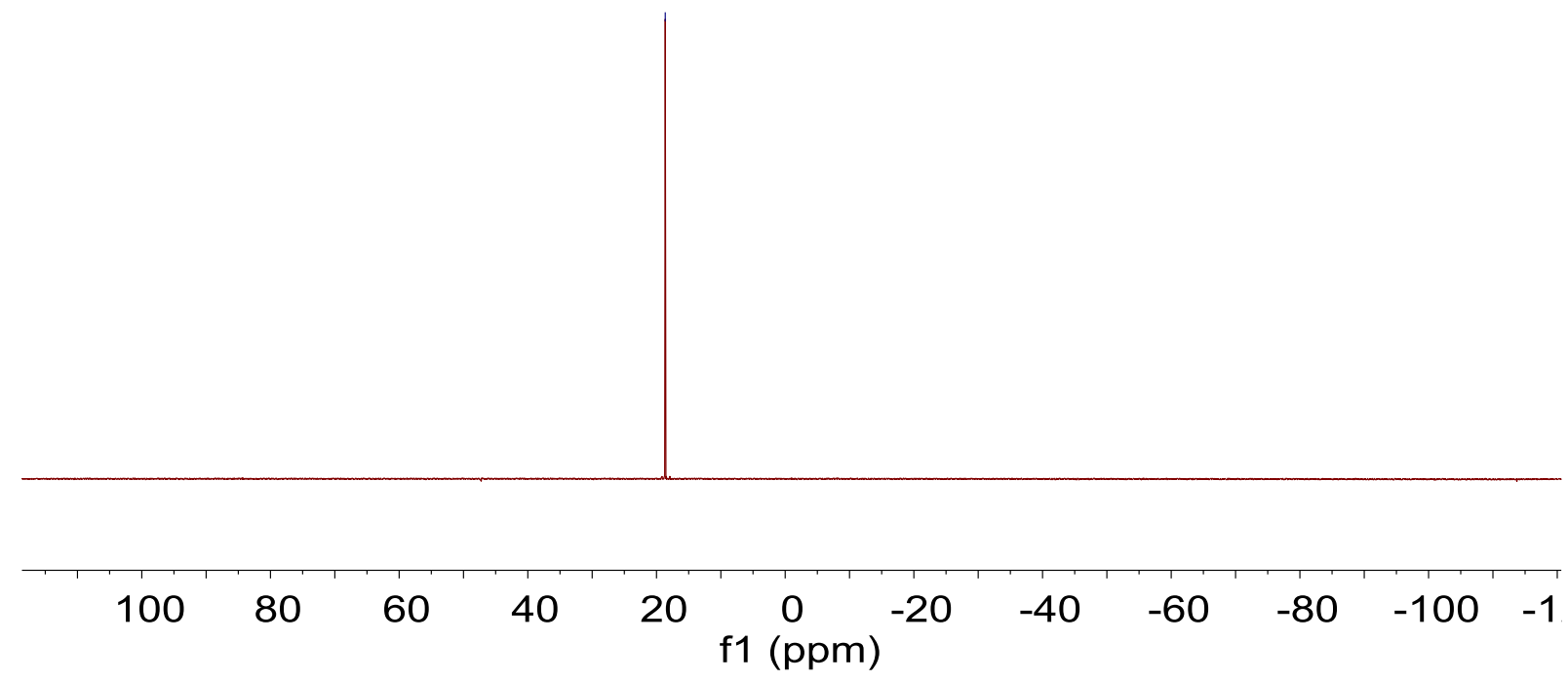


ูํํㅇำ

NNN
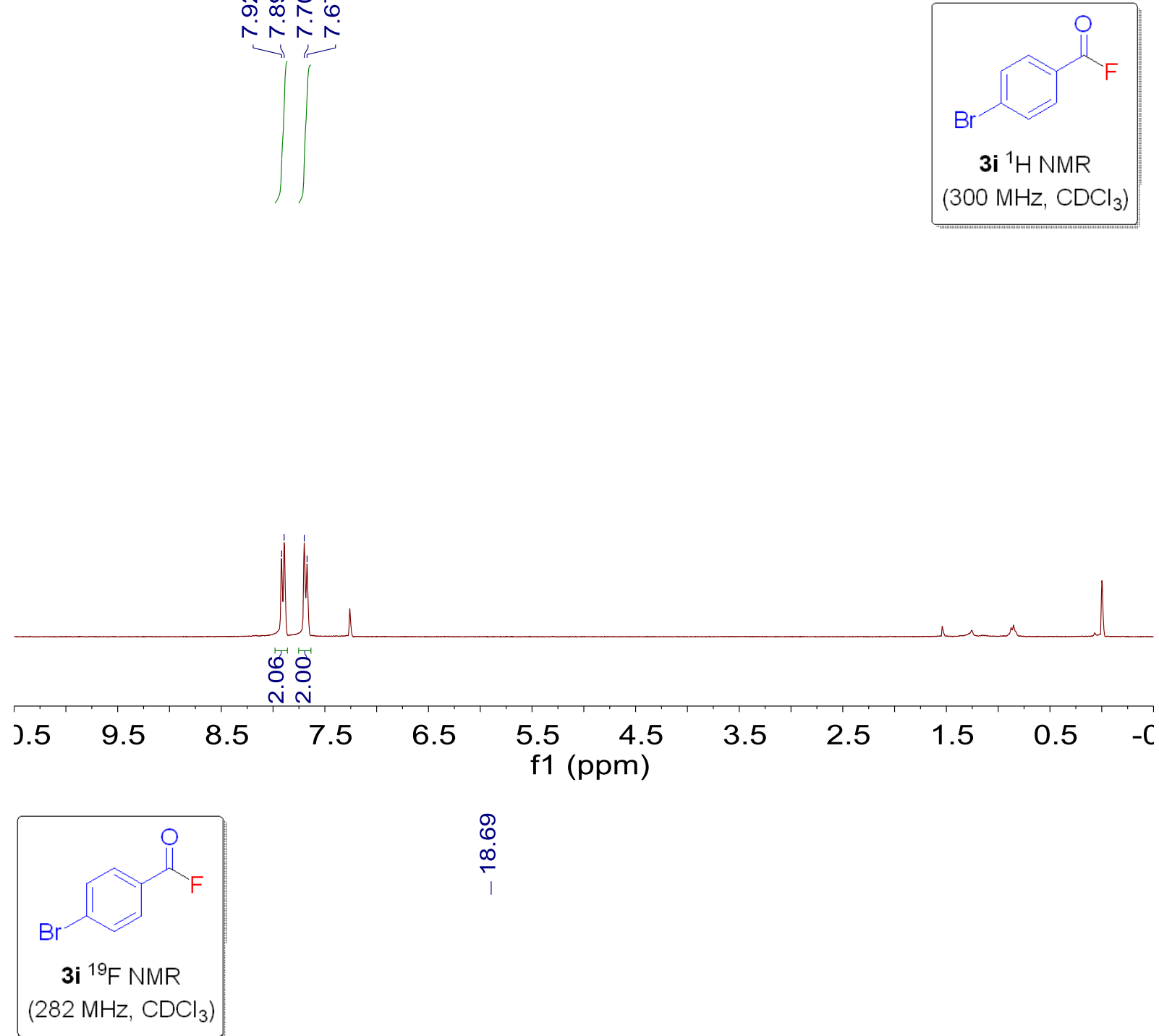

\section{\begin{tabular}{l}
8 \\
0 \\
$\infty$ \\
\hline
\end{tabular}}

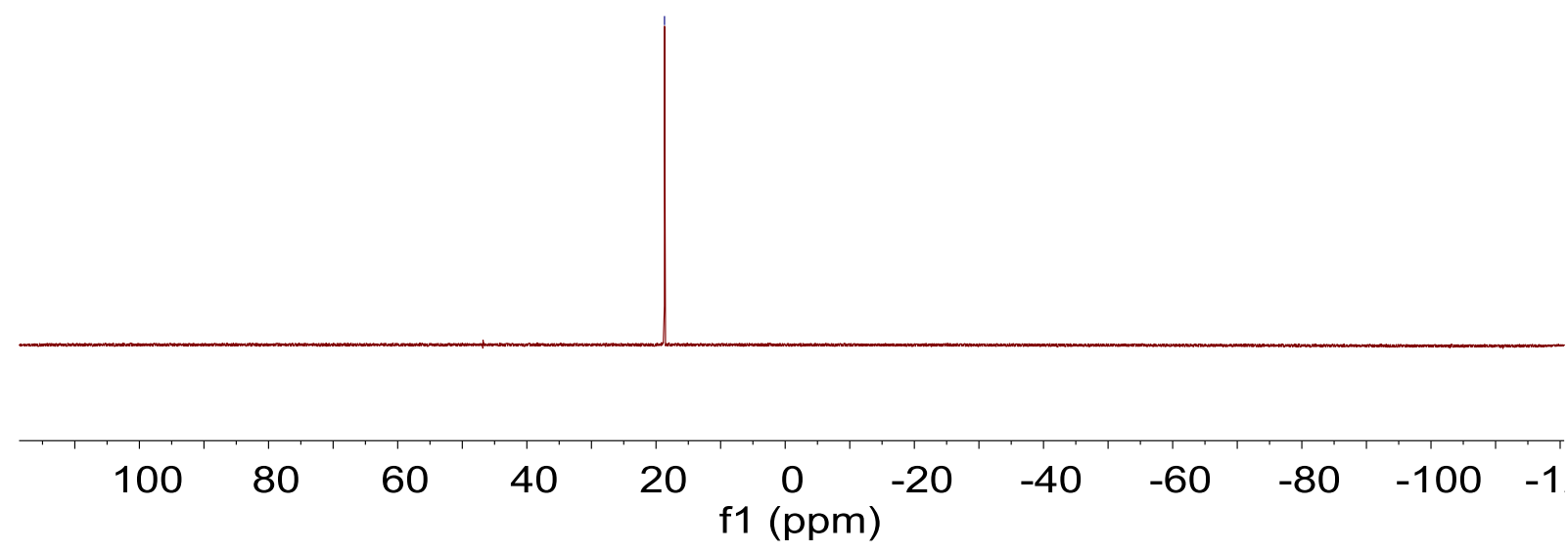



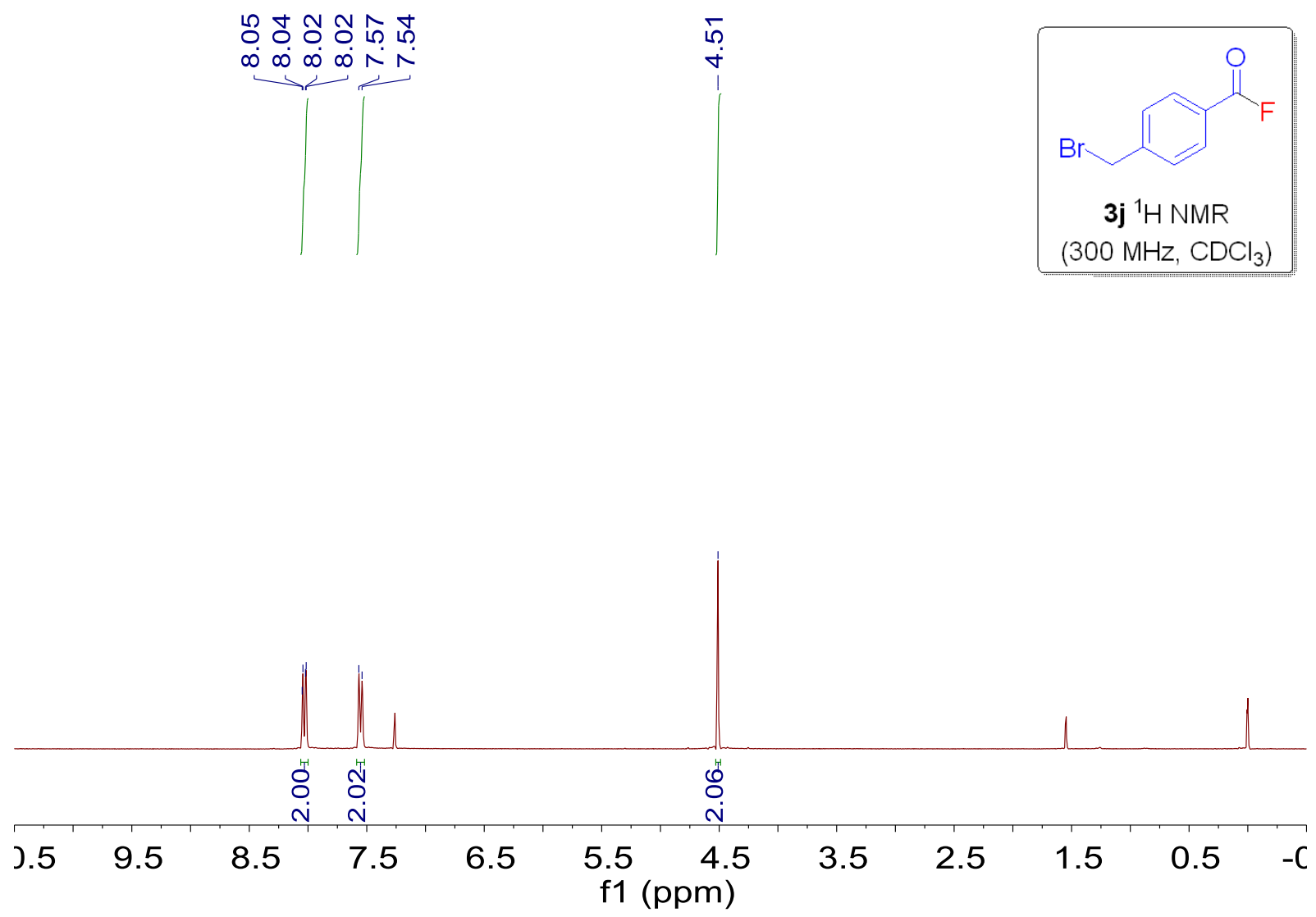

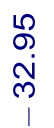
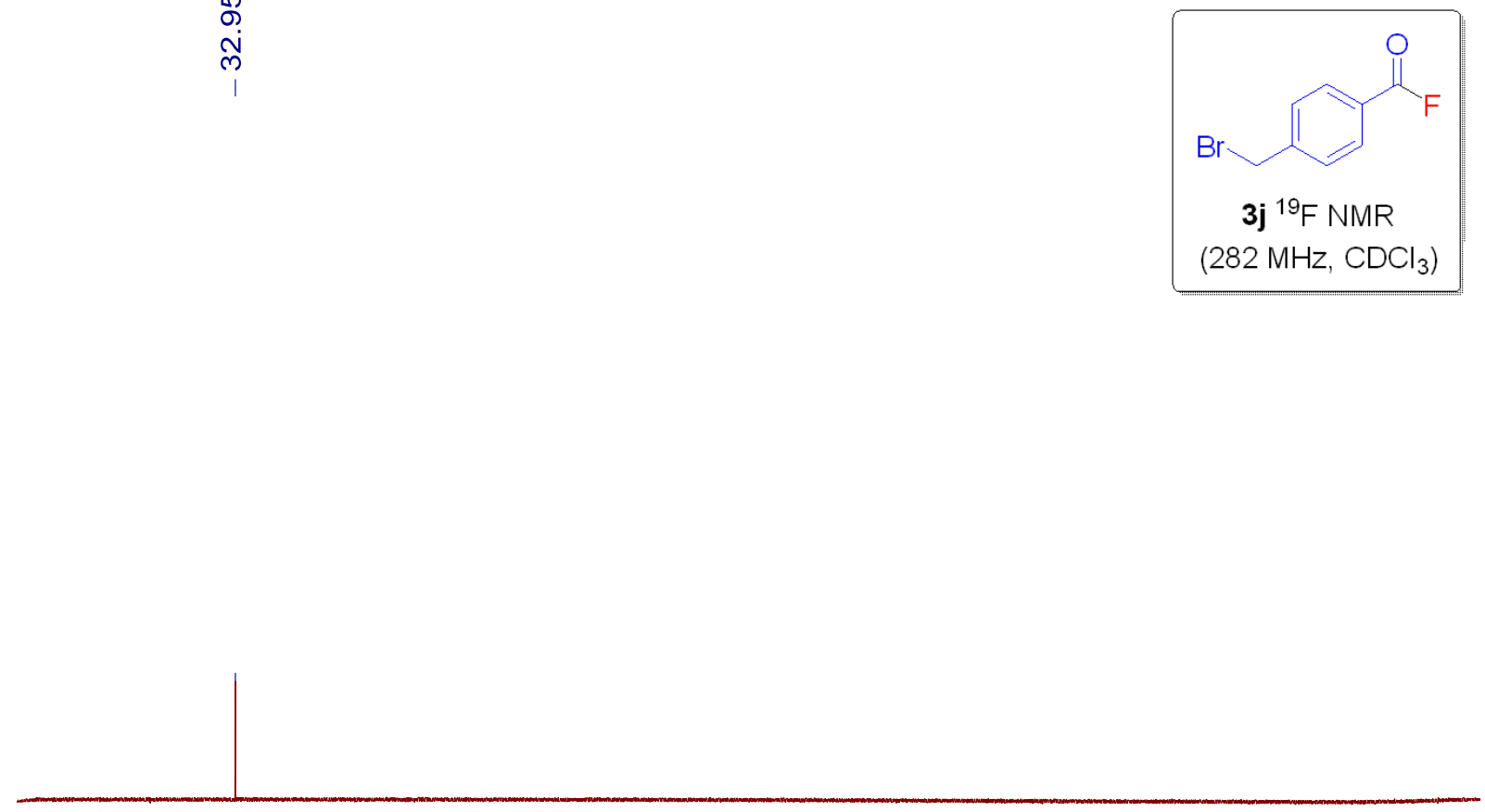

$\begin{array}{llllllllllll}50 & 30 & 10 & -10 & -30 & \begin{array}{l}-50 \\ \mathrm{f} 1\end{array}(\mathrm{ppm}) & -70 & -90 & -110 & -130 & -150 & -1\end{array}$




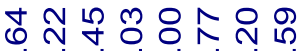

品
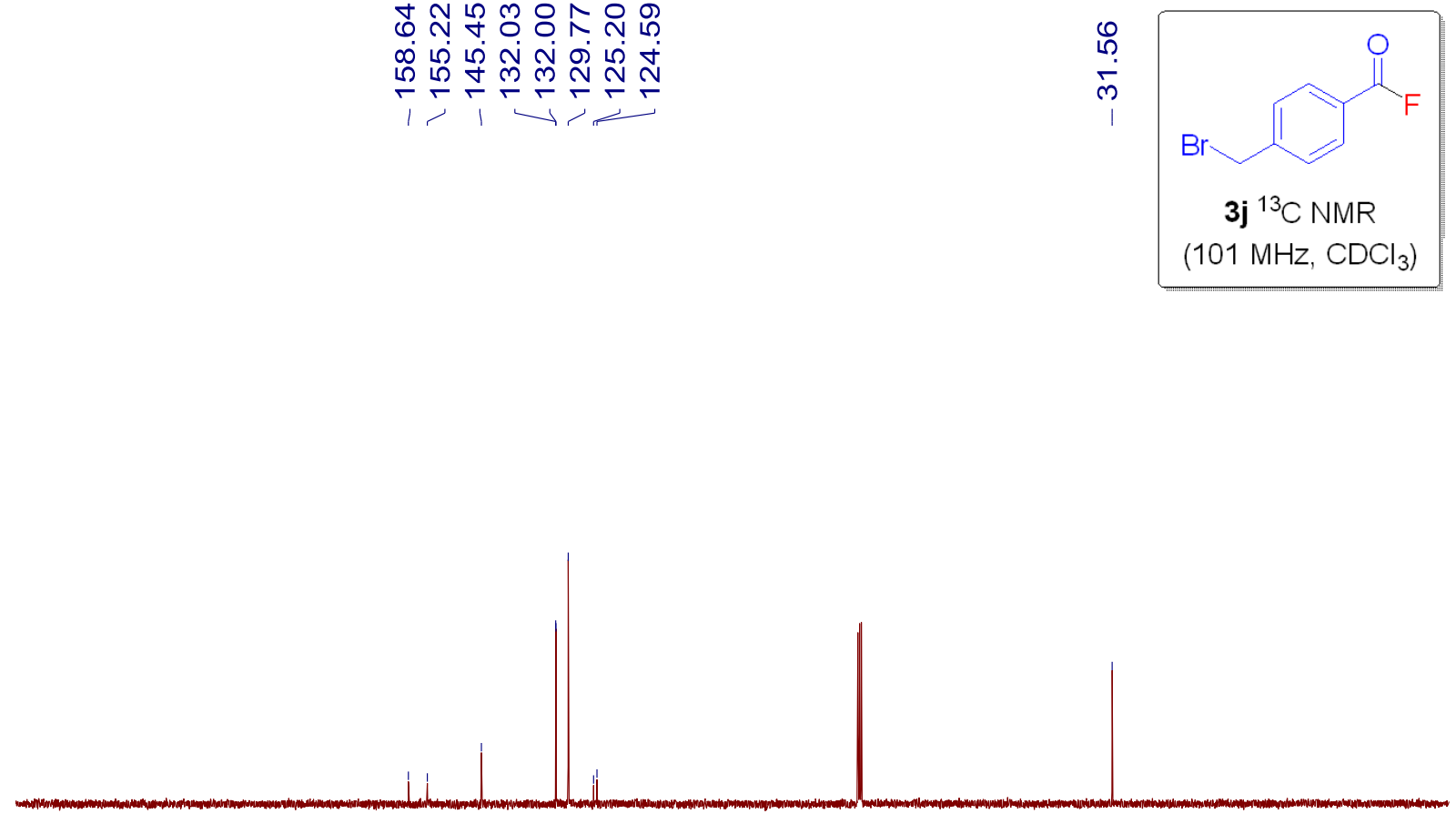

\begin{tabular}{llllllllllll}
\hline 220 & 200 & 180 & 160 & 140 & $120 \underset{\mathrm{f} 1(\mathrm{ppm})}{100} 80$ & 60 & 40 & 20 & 0 & -20
\end{tabular}
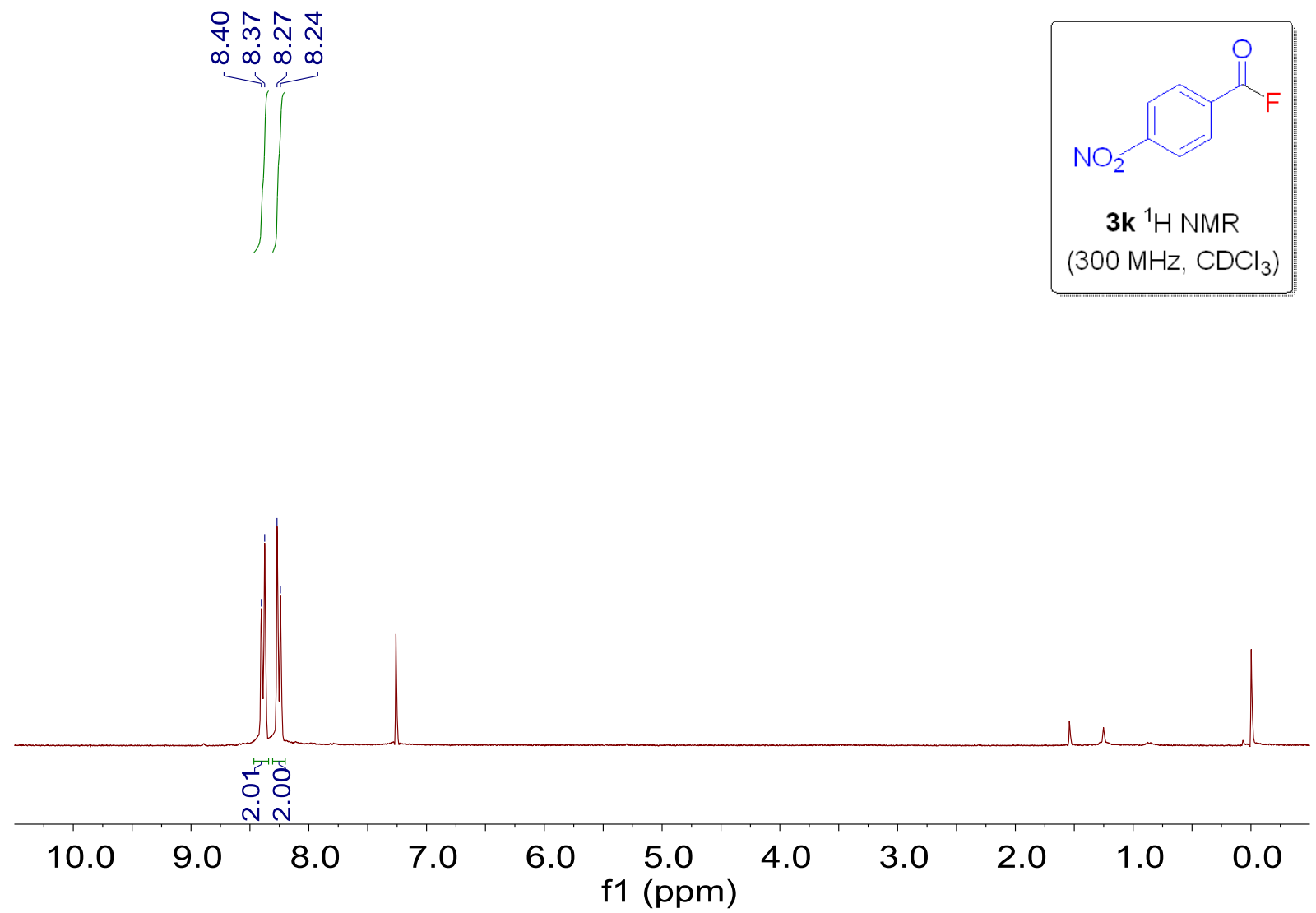


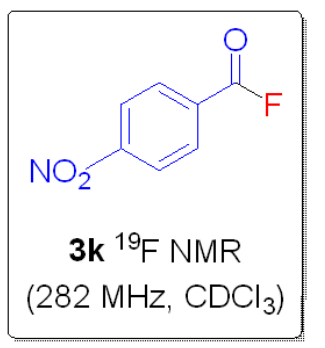

ํำ
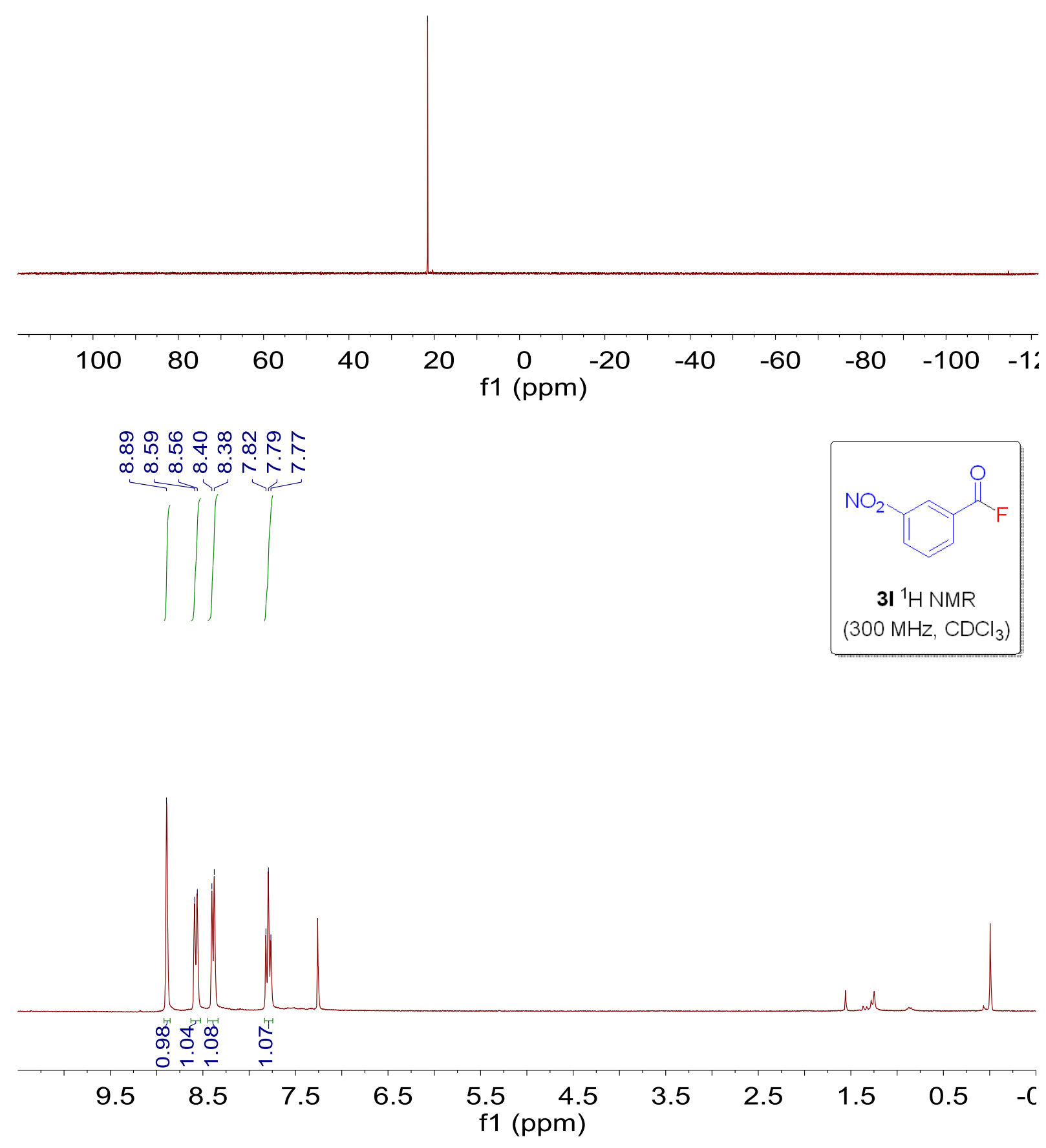


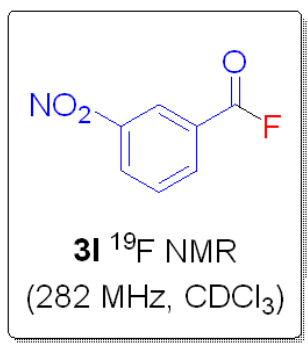

ֻั

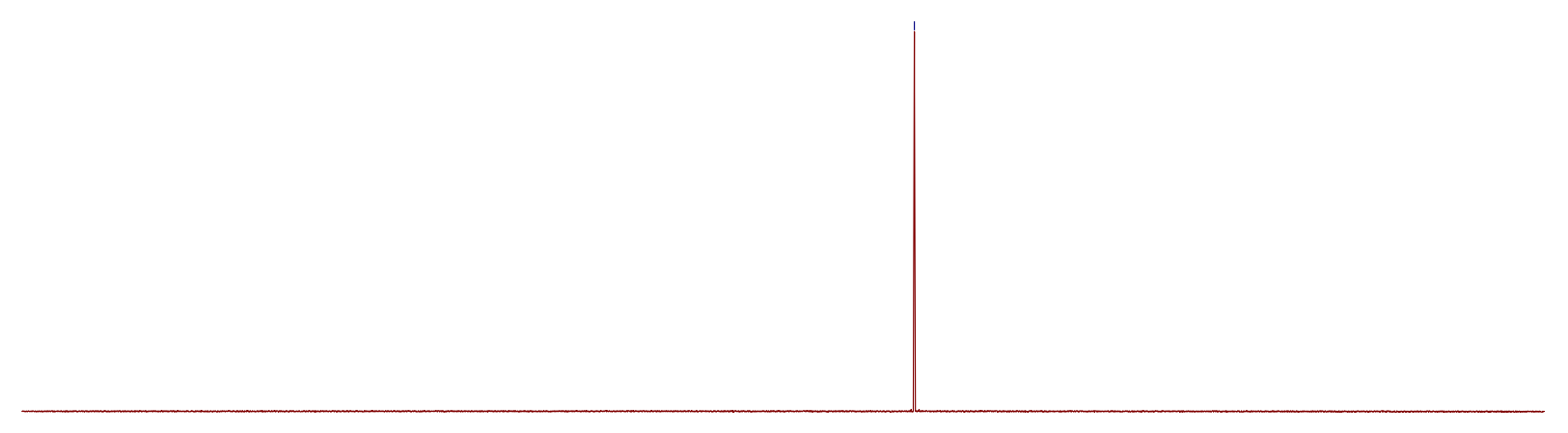

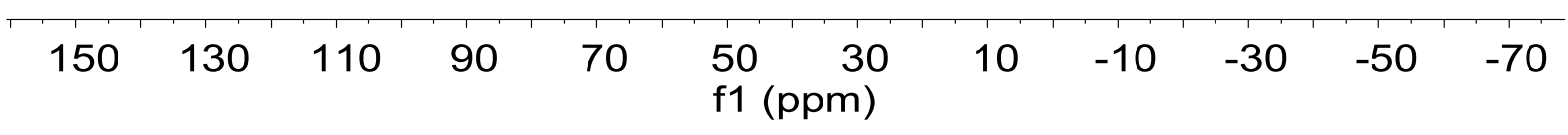

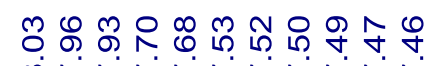

$\infty N \wedge N \wedge N \wedge \wedge$
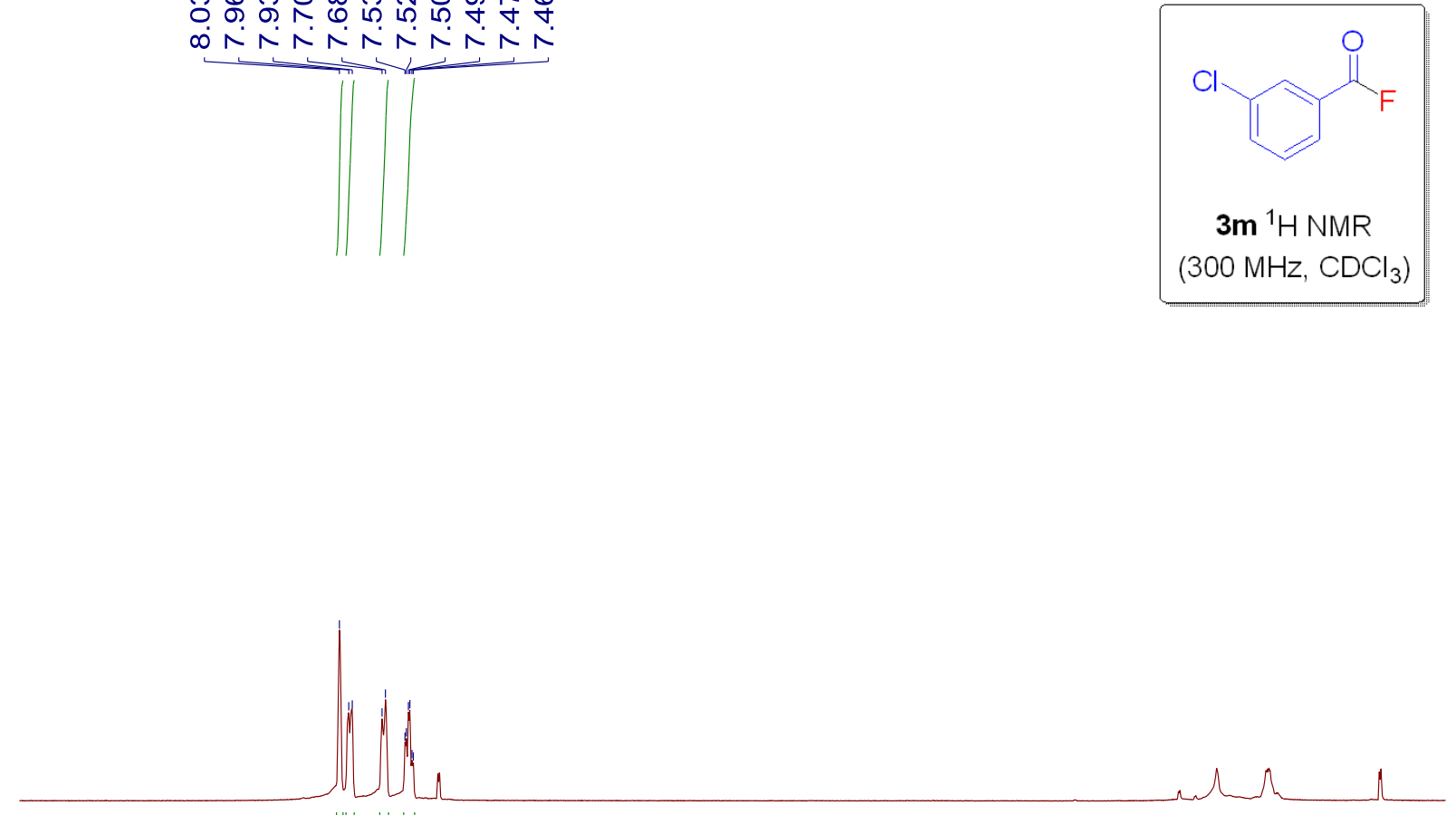

80

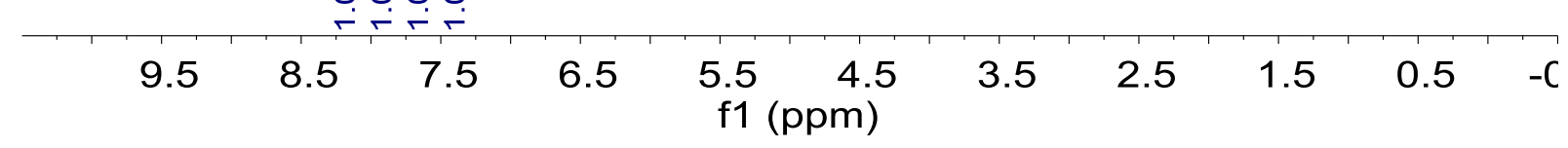



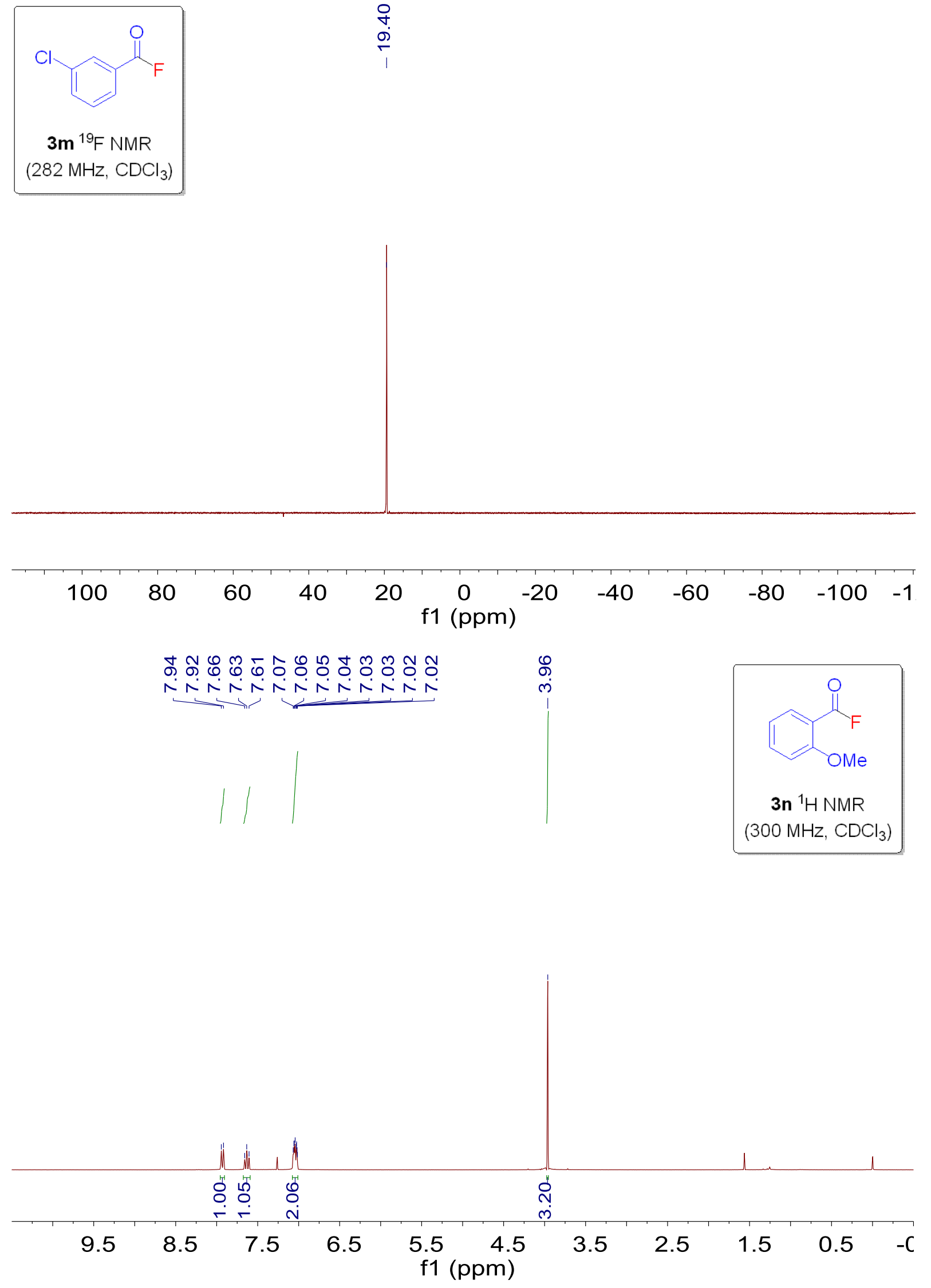


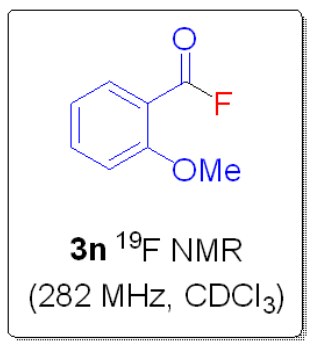

吕

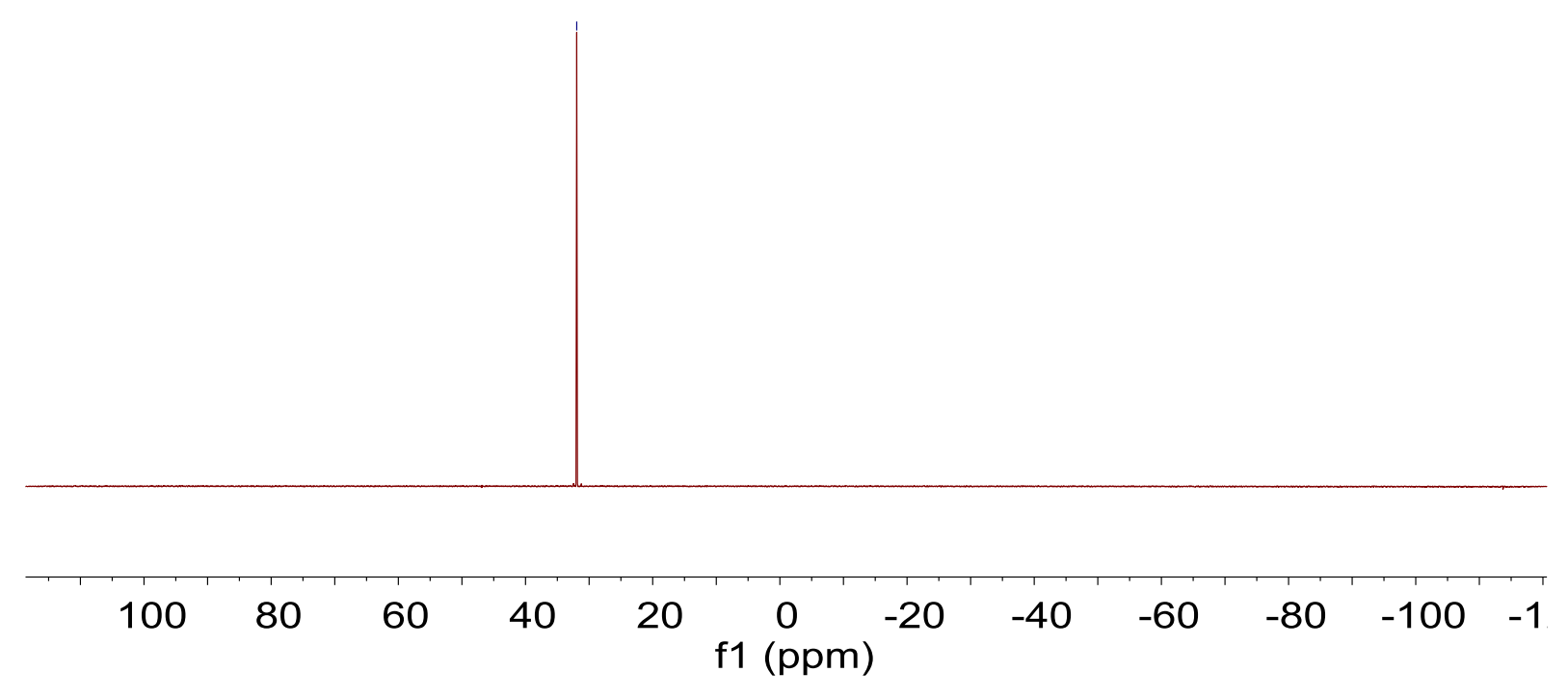

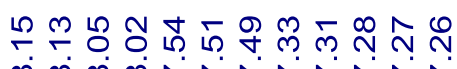

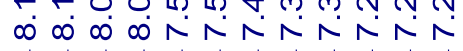
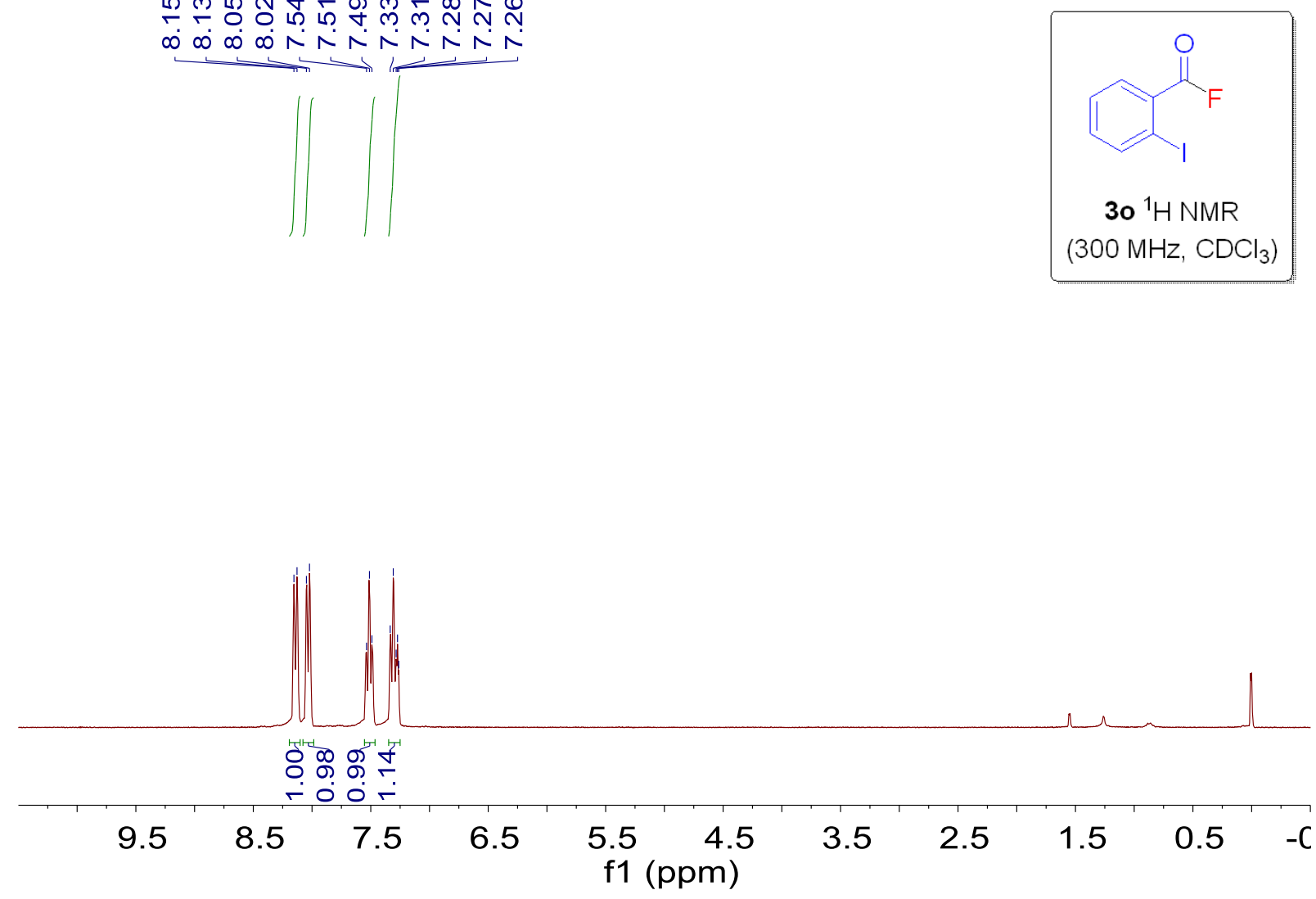


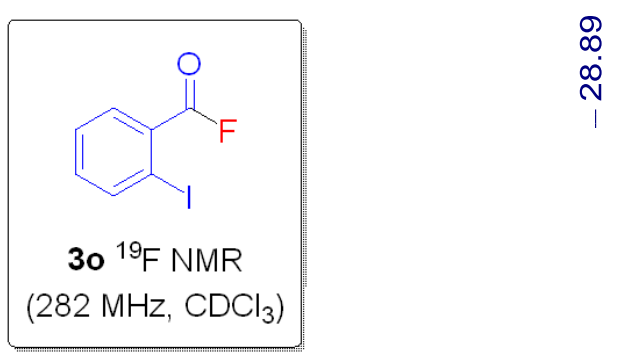

\begin{tabular}{|c|c|c|c|c|c|c|c|c|c|c|c|}
\hline 100 & 80 & 60 & 40 & 20 & $\begin{array}{c}0 \\
\mathrm{f} 1(\mathrm{ppm}\end{array}$ & )$^{-20}$ & -40 & -60 & -80 & -100 & -1 \\
\hline
\end{tabular}

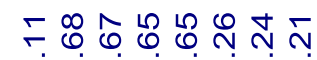

$\infty N N N N$
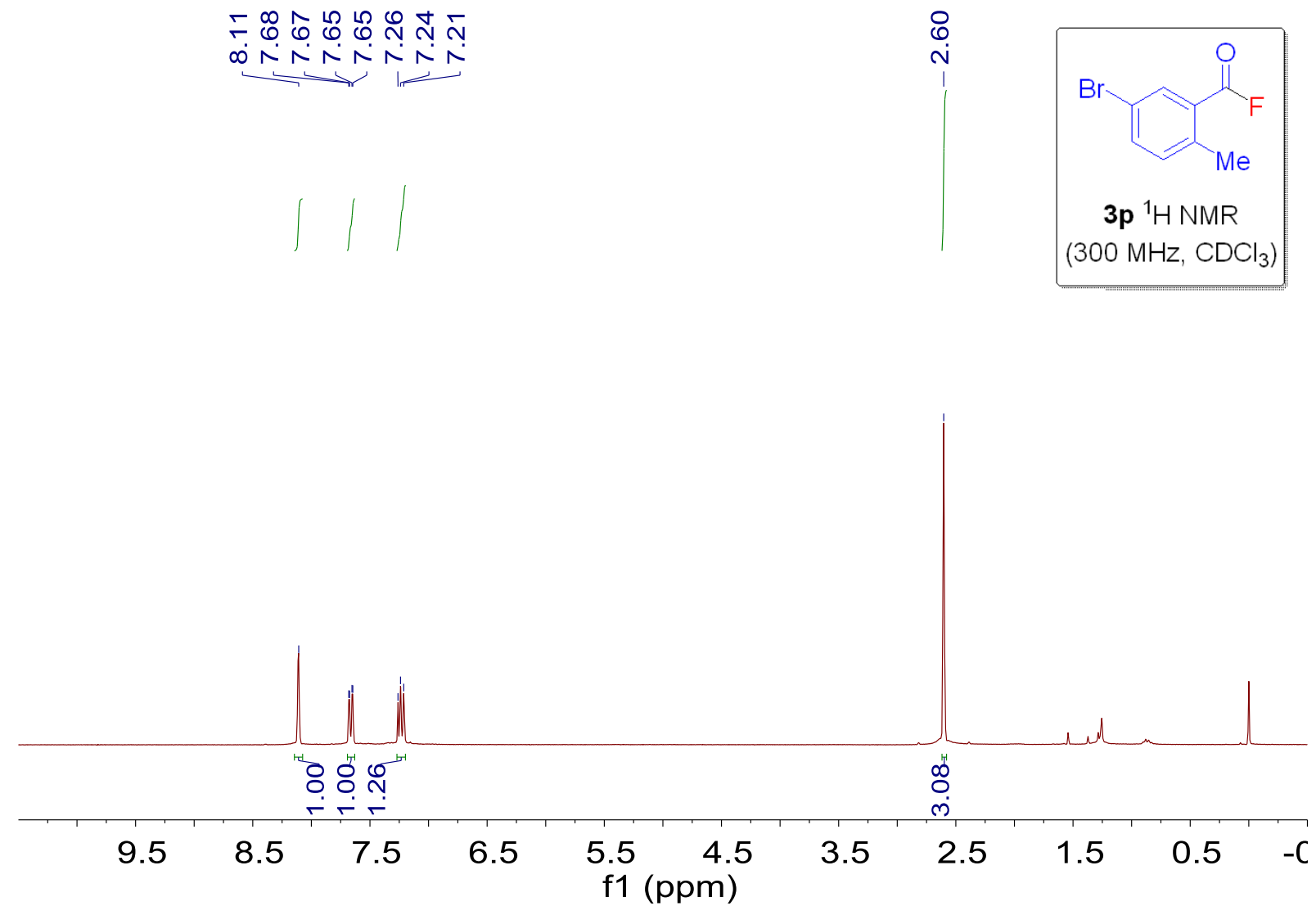


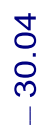

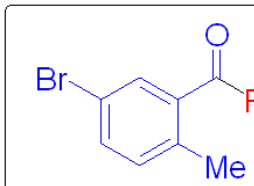

$3{ }^{19} \mathrm{~F}$ NMR

$\left(282 \mathrm{MHz}, \mathrm{CDCl}_{3}\right)$

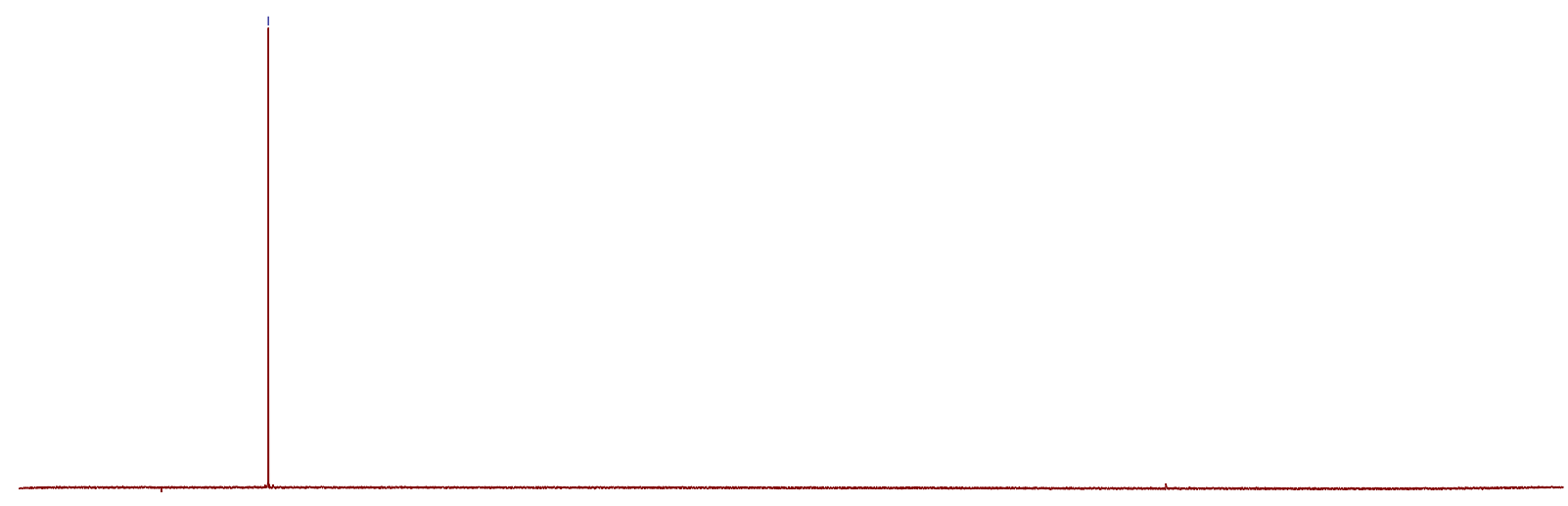

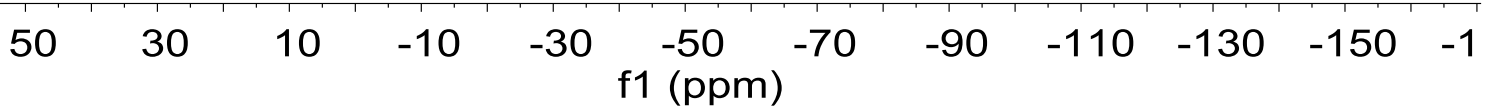

ஸุำ

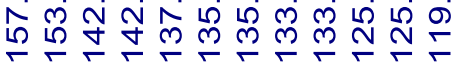

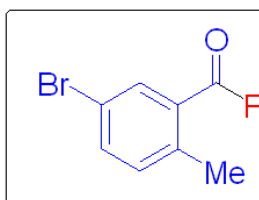

$3{ }^{13} \mathrm{C} \mathrm{NMR}$

$\left(101 \mathrm{MHz}, \mathrm{CDCl}_{3}\right.$ )

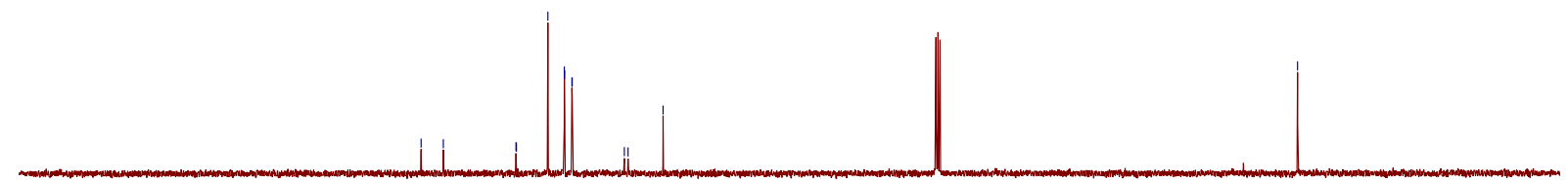

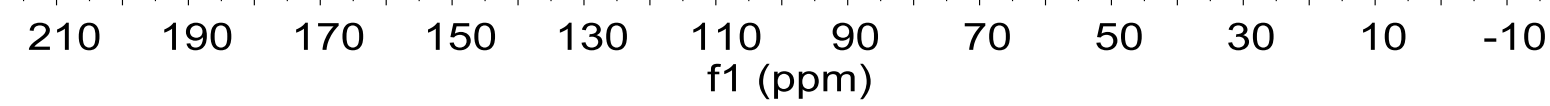



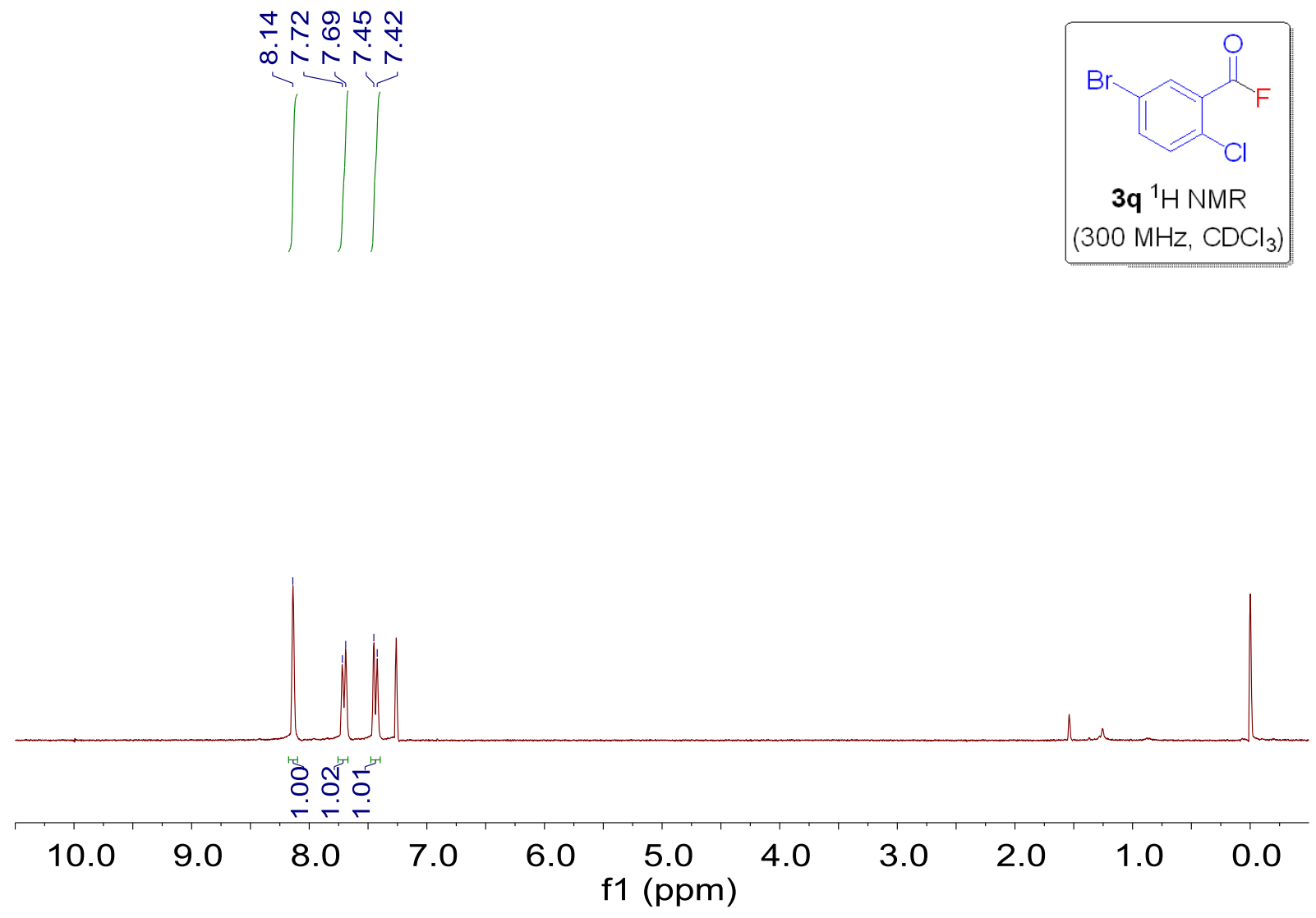

N
N
m
1

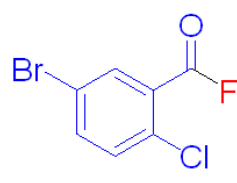

$3 q{ }^{19} \mathrm{~F} \mathrm{NMR}$

$\left(376 \mathrm{MHz}, \mathrm{CDCl}_{3}\right)$

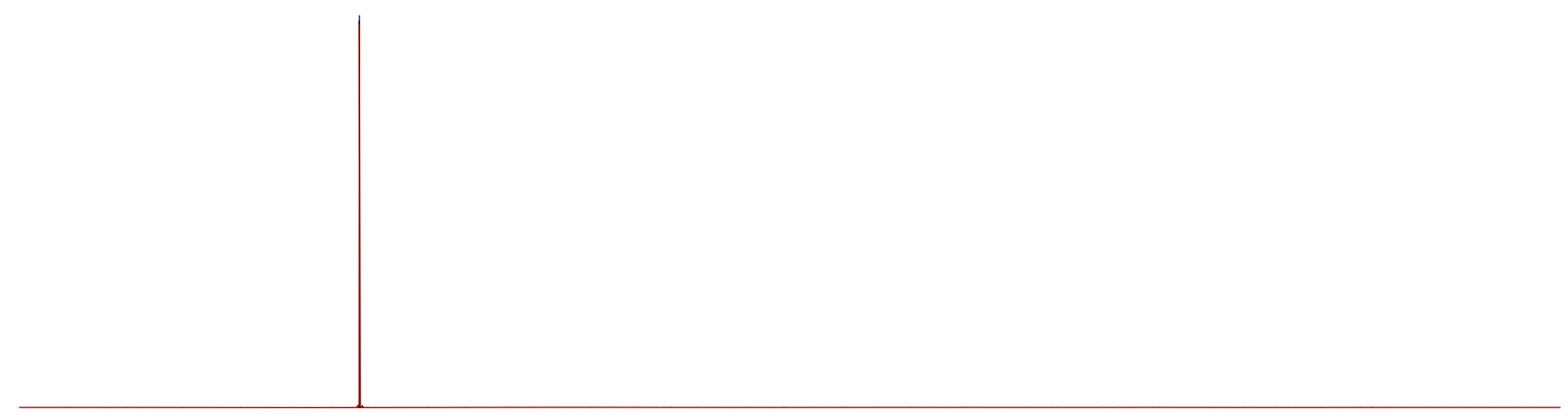

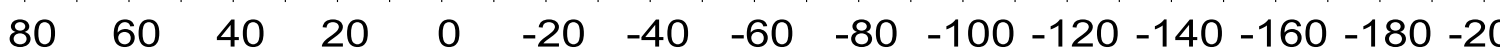
f1 (ppm) 


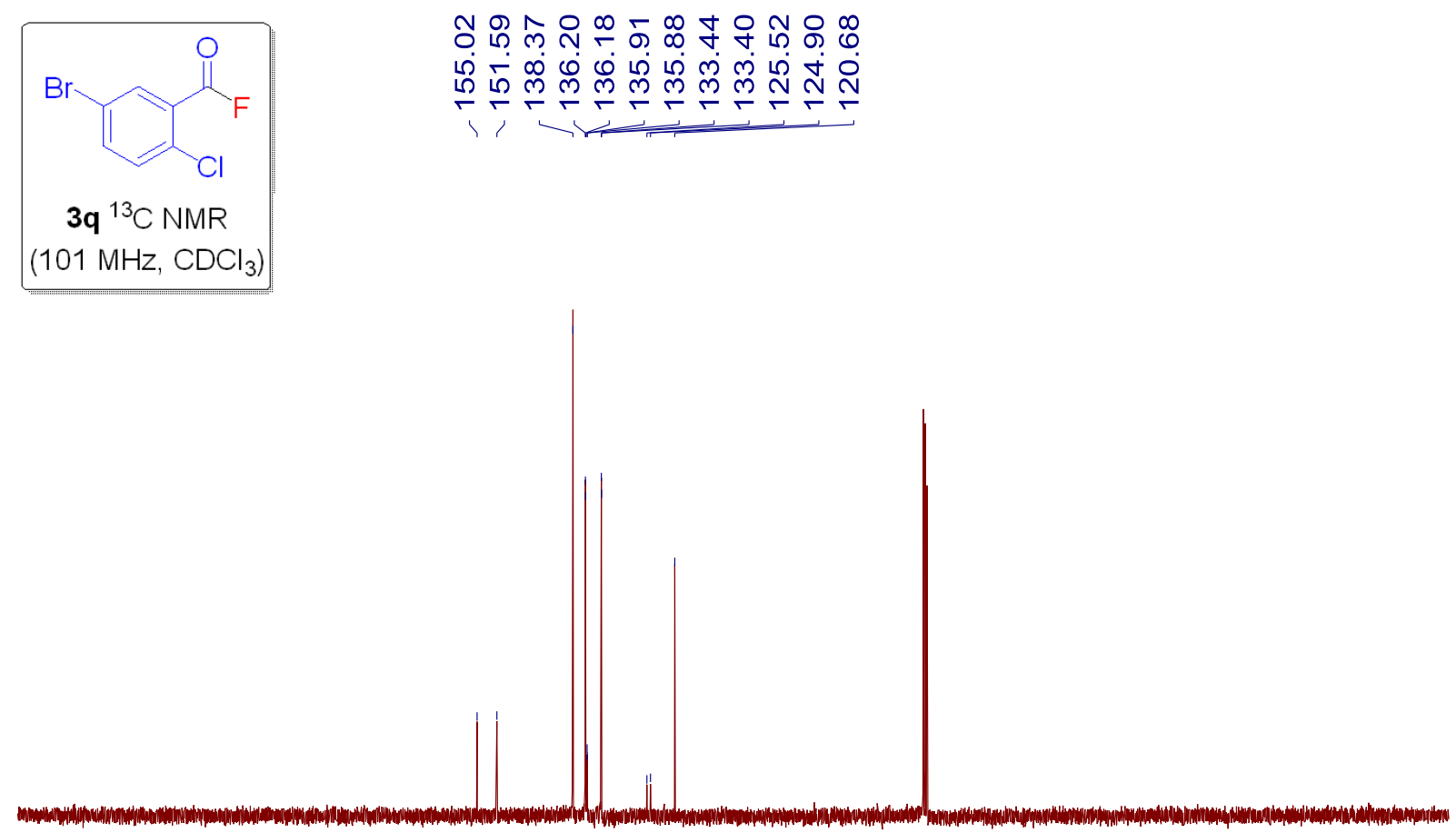

\begin{tabular}{|c|c|c|c|c|c|c|c|c|c|c|c|c|}
\hline 230 & 210 & 190 & 170 & 150 & 130 & $\begin{array}{l}110 \\
(\mathrm{ppm})\end{array}$ & 90 & 70 & 50 & 30 & 10 & -1( \\
\hline
\end{tabular}
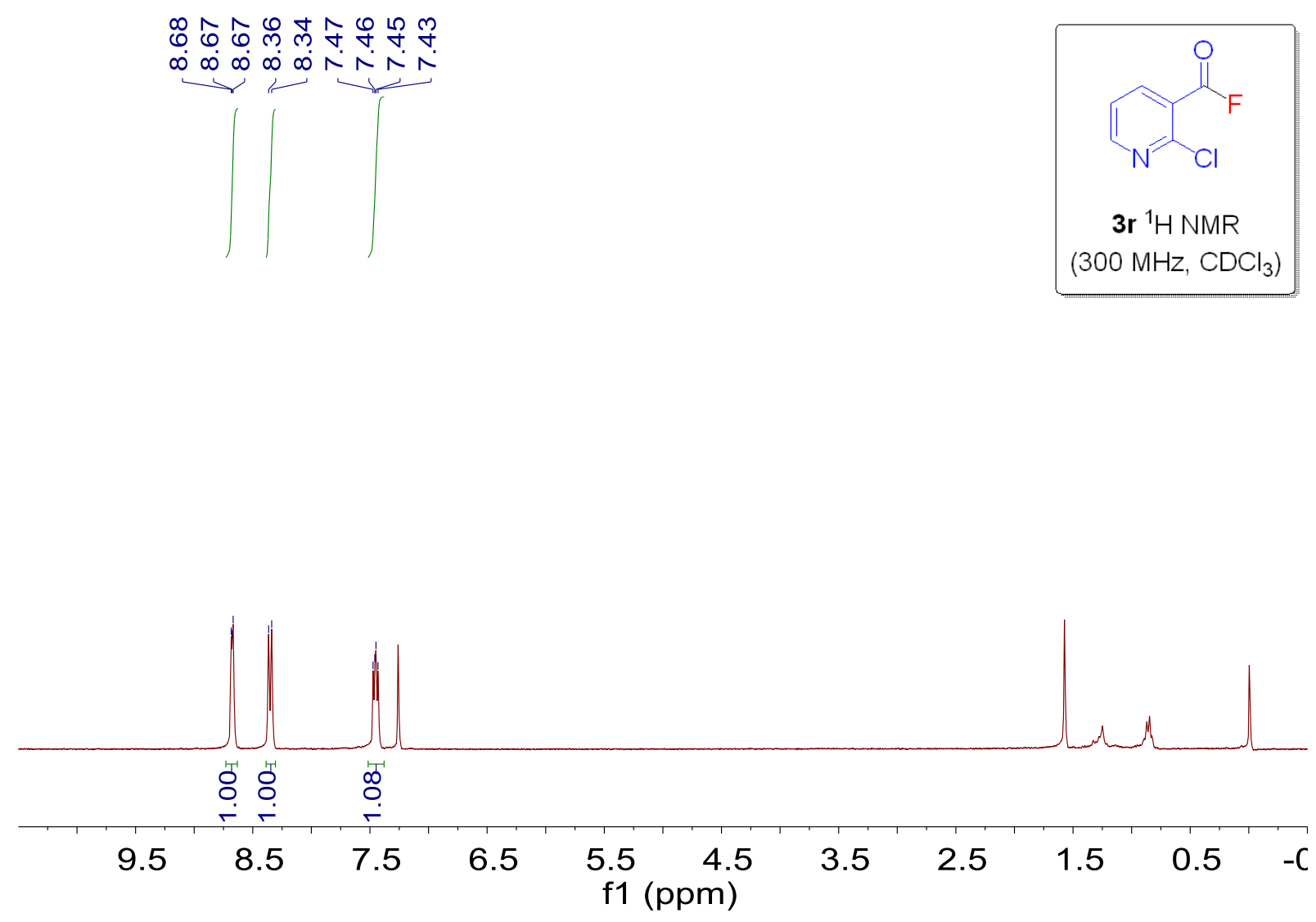


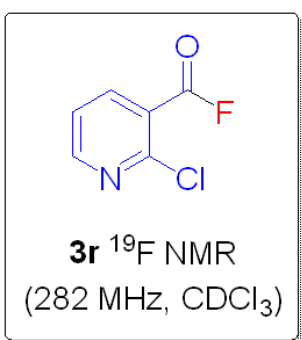

\section{$\infty$}

लें

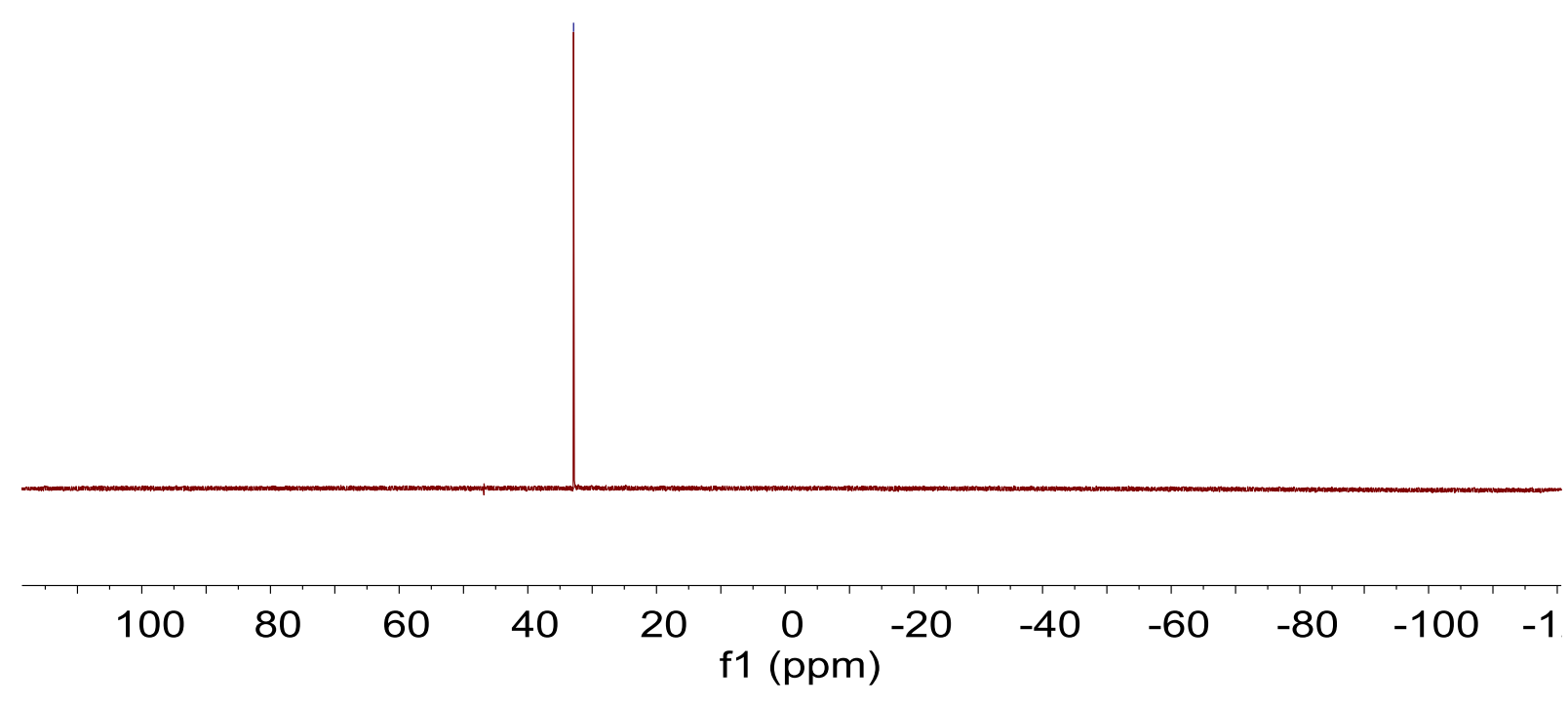

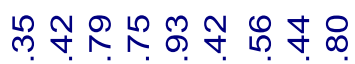

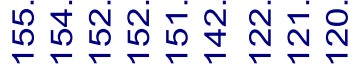

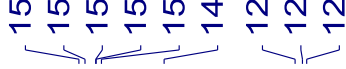

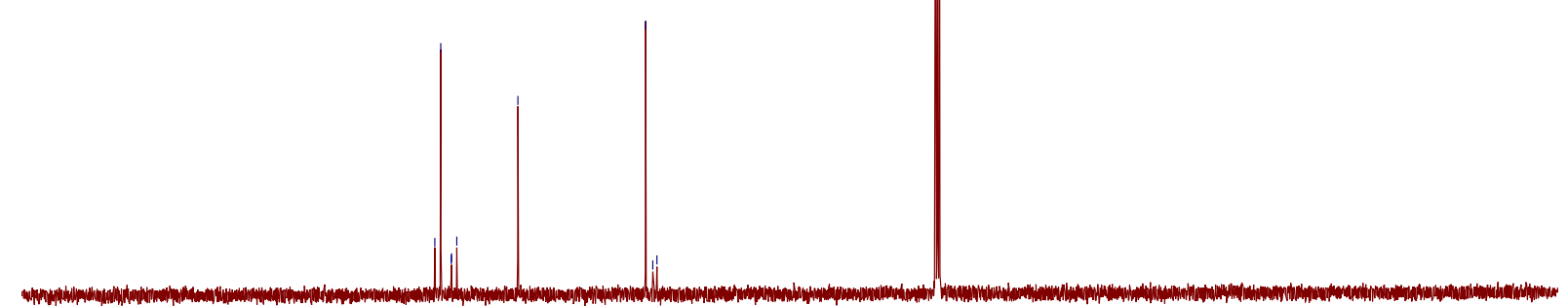

$\begin{array}{llllllllllll}210 & 190 & 170 & 150 & 130 & \begin{array}{c}110 \\ \mathrm{f} 1(\mathrm{ppm})\end{array} & 90 & 70 & 50 & 30 & 10 & -10\end{array}$




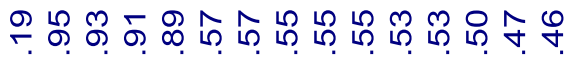

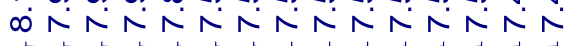
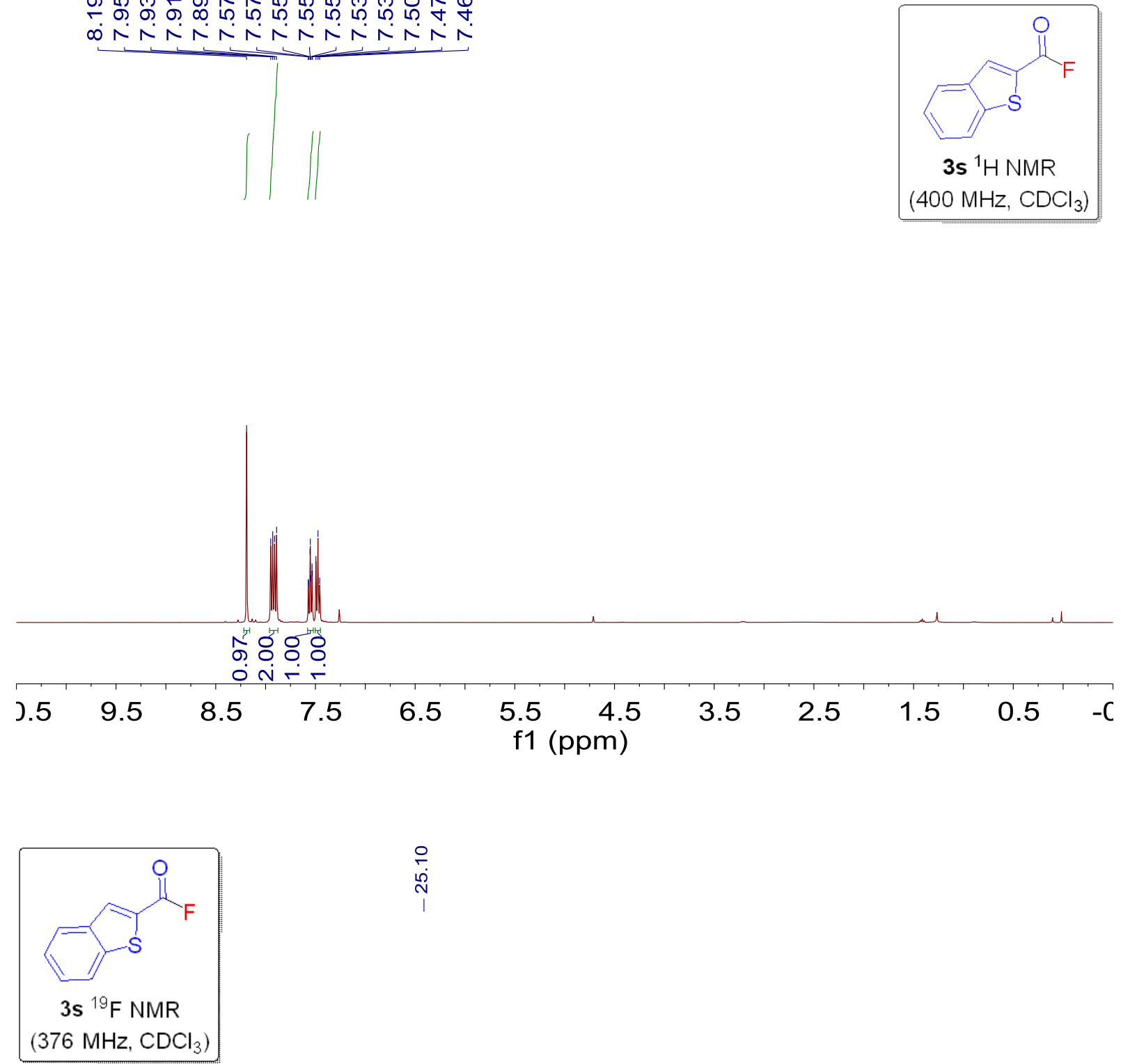

올

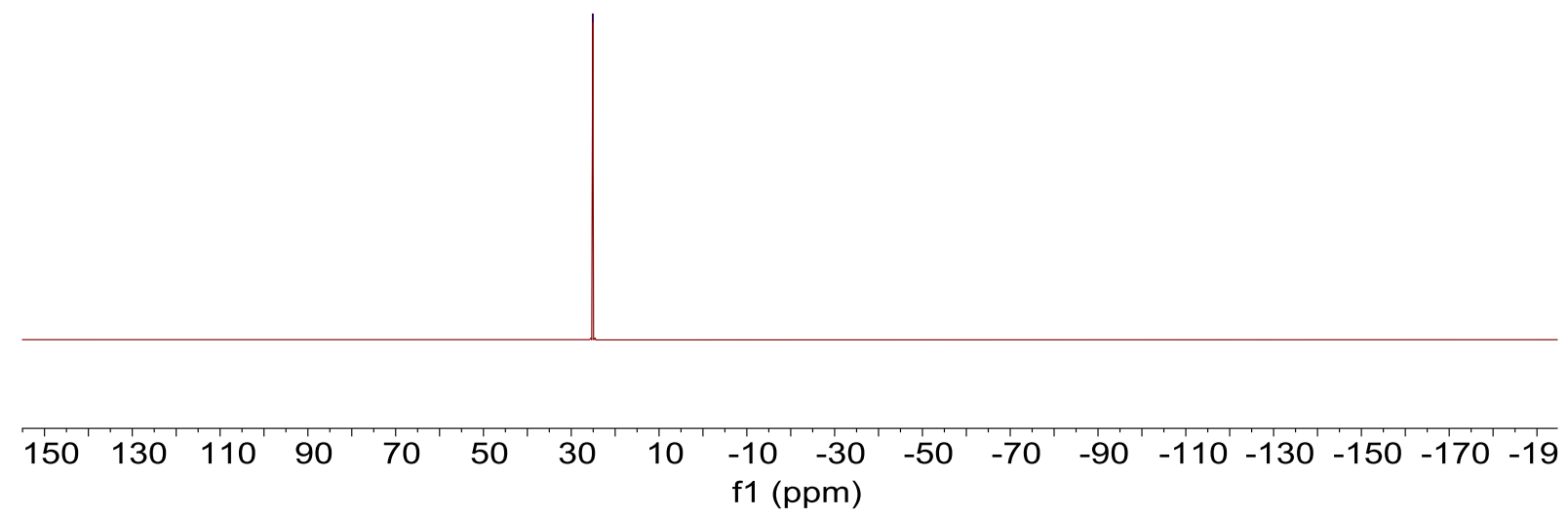




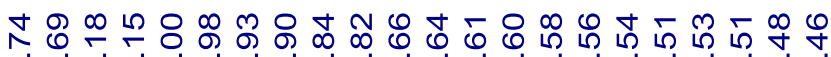

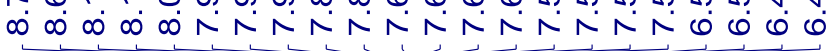

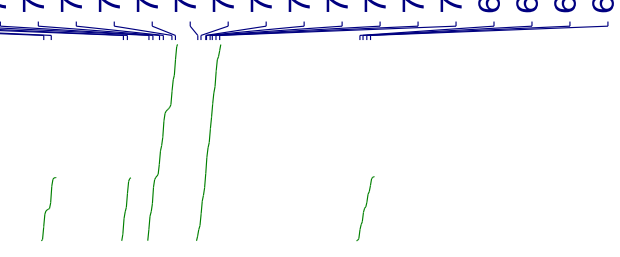

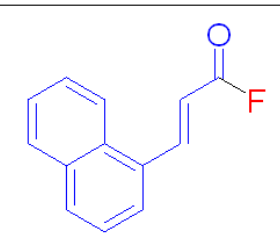

3t ${ }^{1} \mathrm{H}$ NMR

$\left(300 \mathrm{MHz}, \mathrm{CDCl}_{3}\right)$
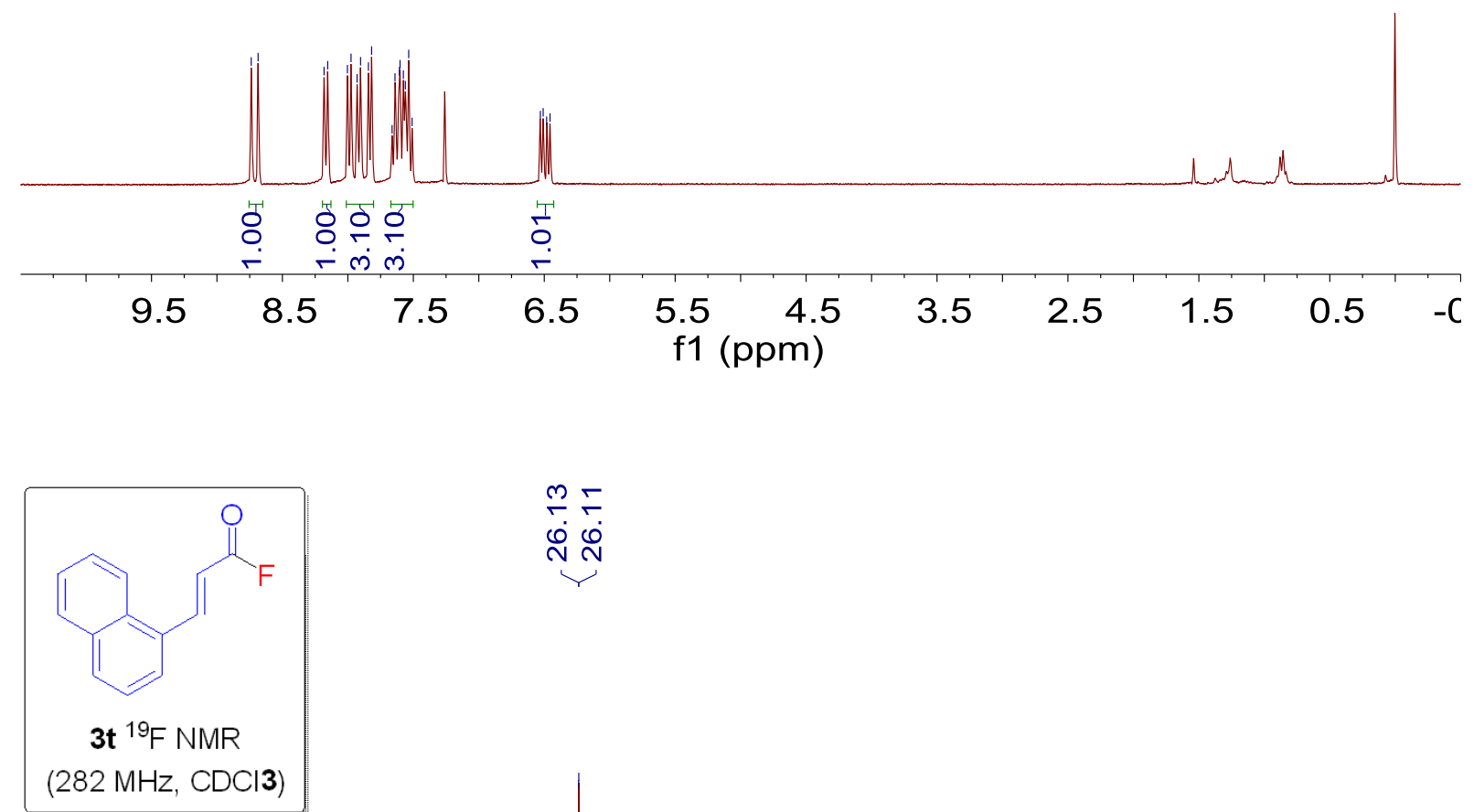

m닫

$\stackrel{\varphi}{N} \stackrel{\circ}{N}$

$\checkmark$

100

$80 \quad 60 \quad 40$

20

$\begin{array}{lllllll}0 & -20 & -40 & -60 & -80 & -100 & -1 \\ (\mathrm{ppm})\end{array}$ 


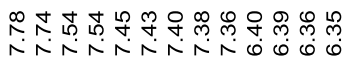

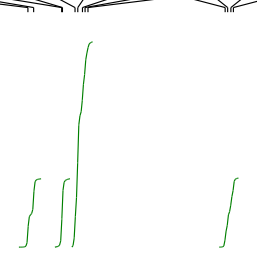

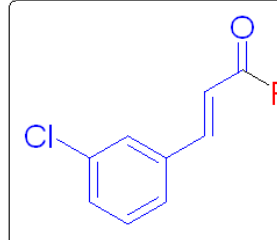

3u ${ }^{1} \mathrm{H}$ NMR

$\left(400 \mathrm{MHz}, \mathrm{CDCl}_{3}\right.$ )

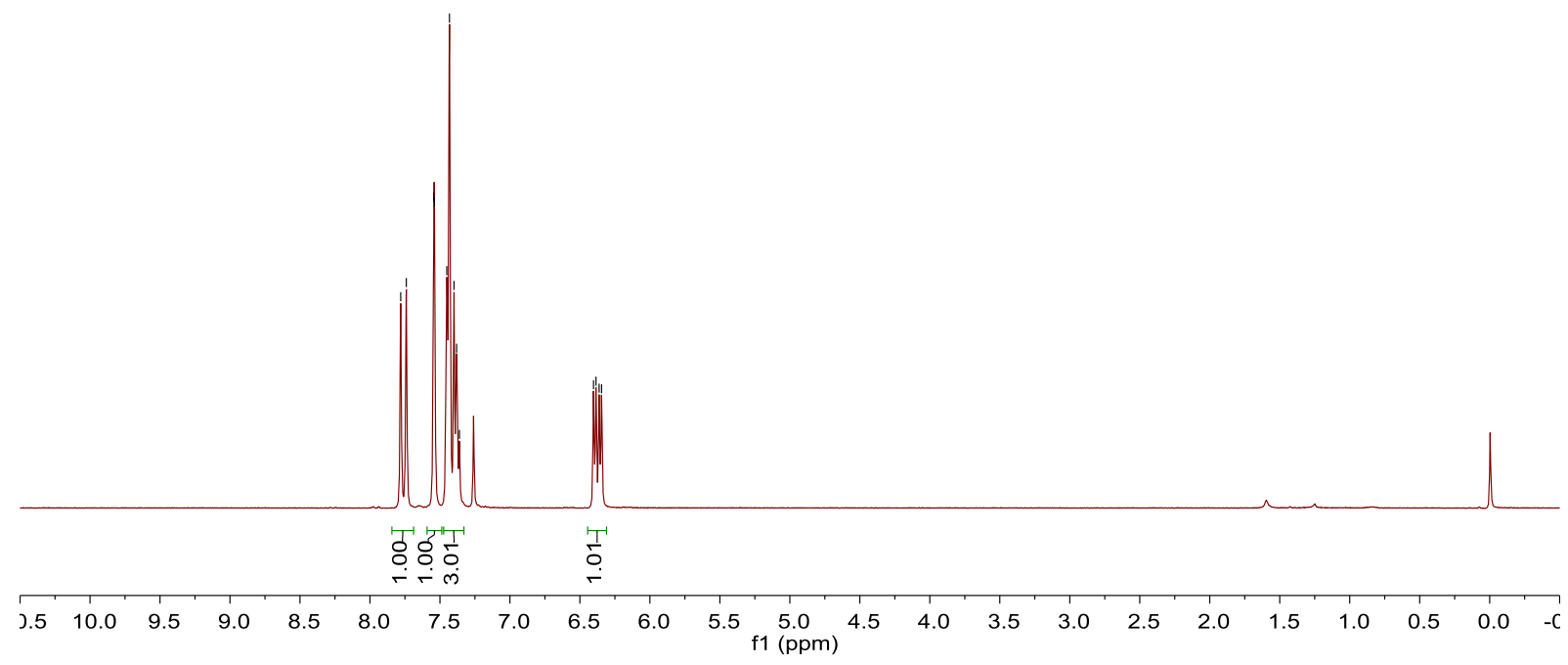

$\underset{\substack{\infty \\ \stackrel{\infty}{\infty}}}{1}$
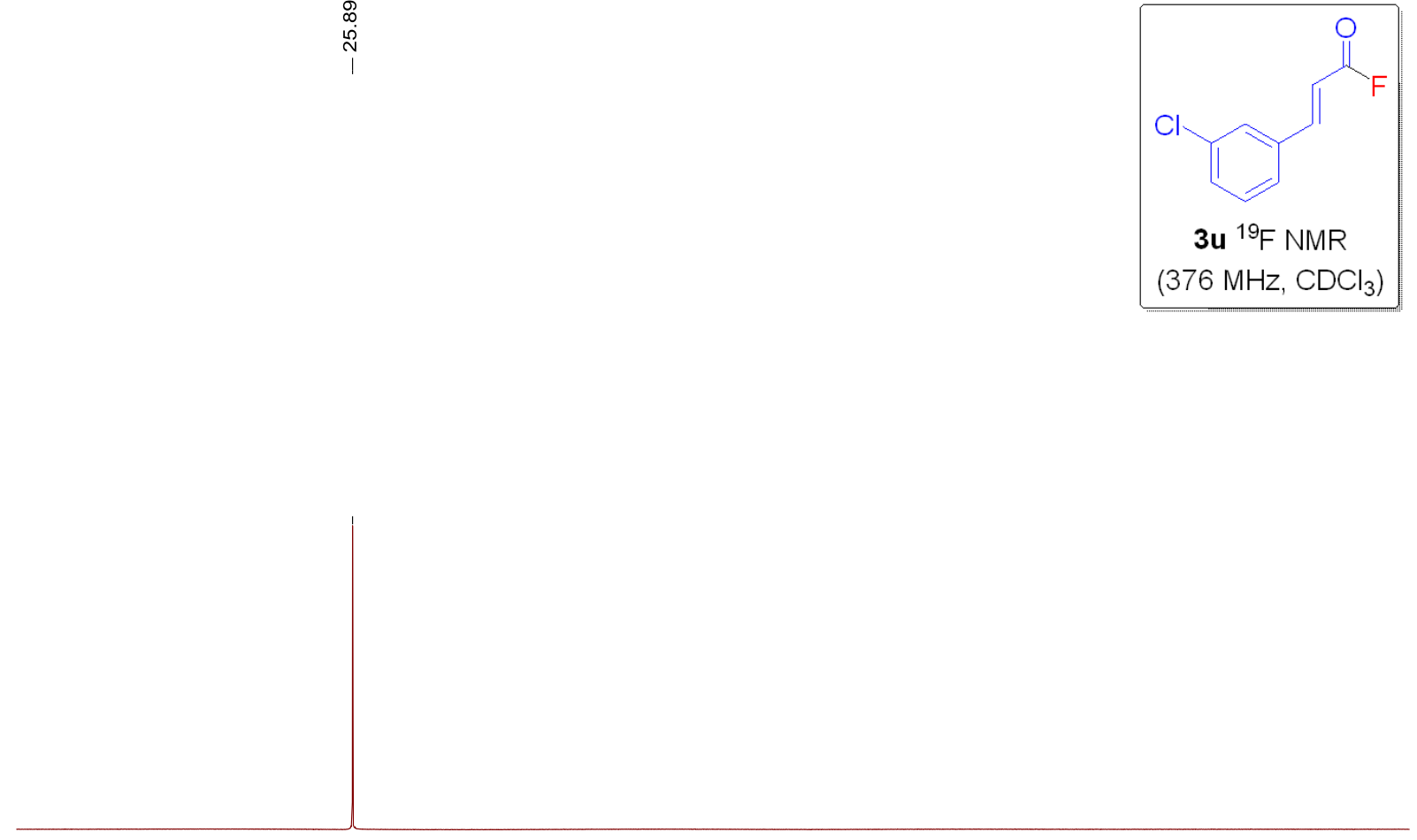

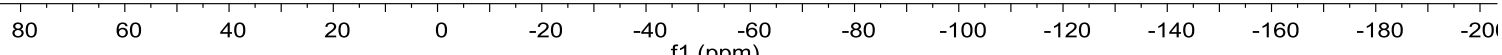




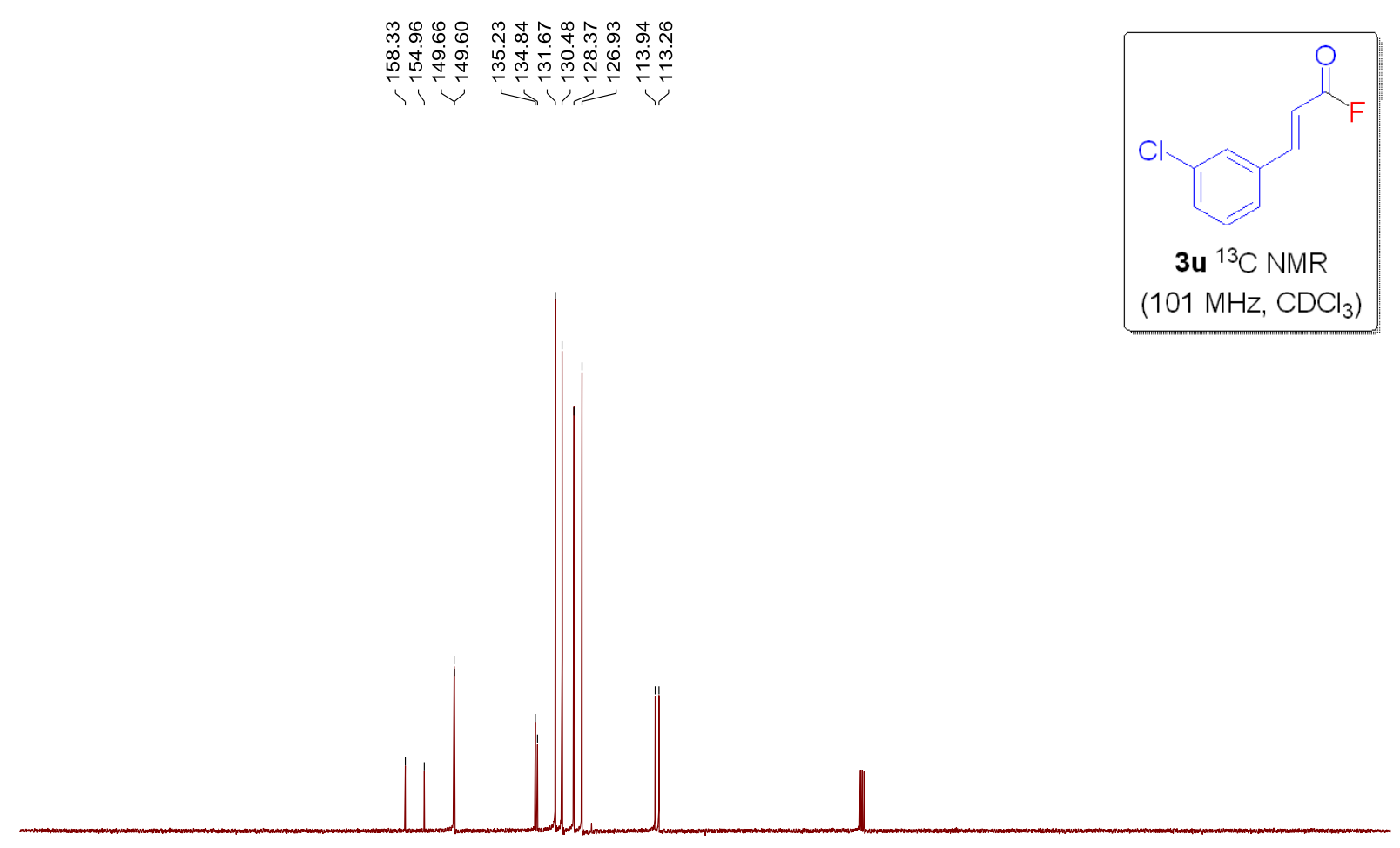

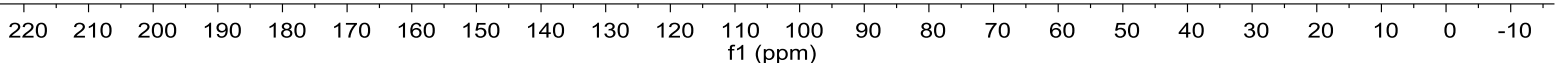
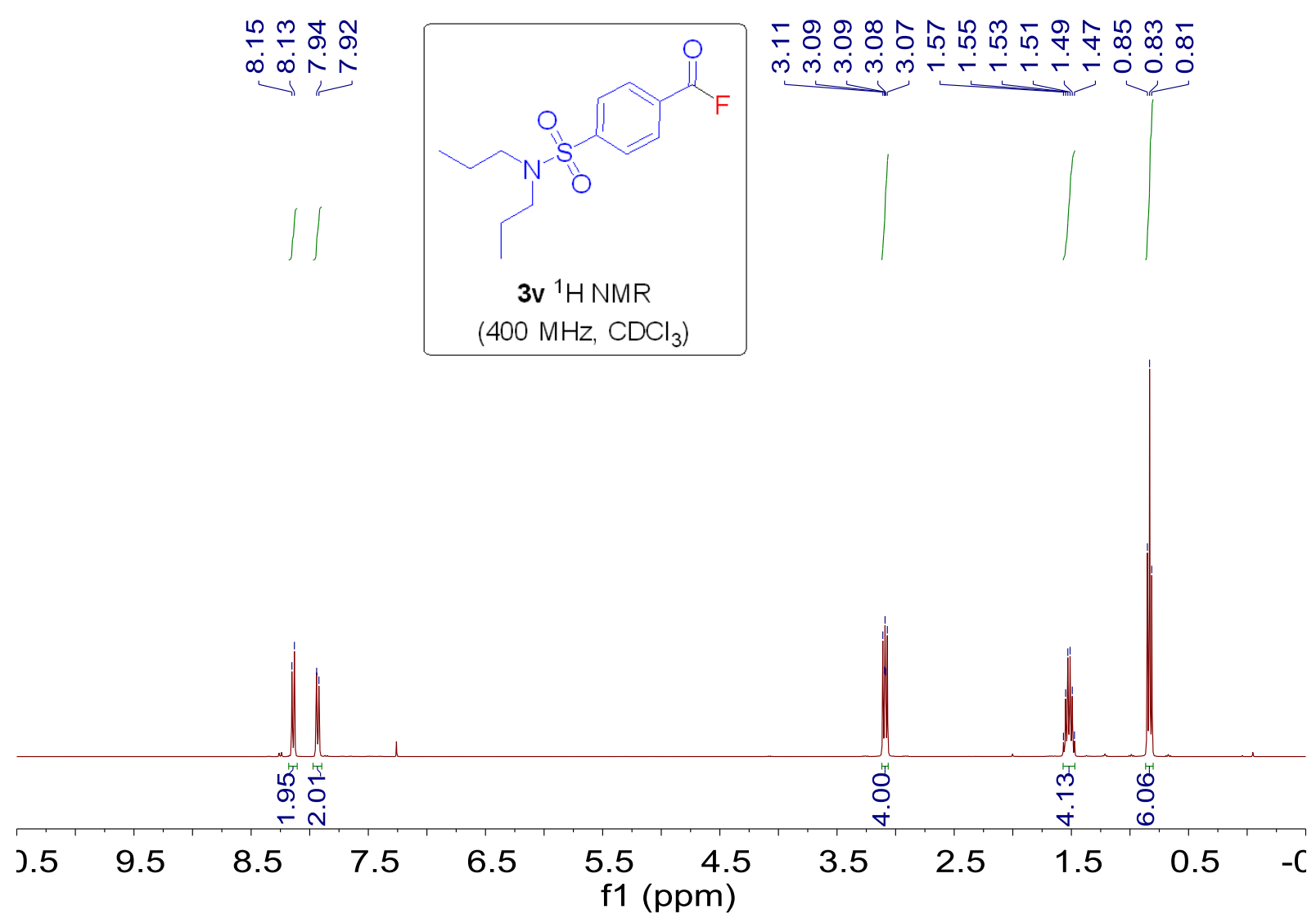


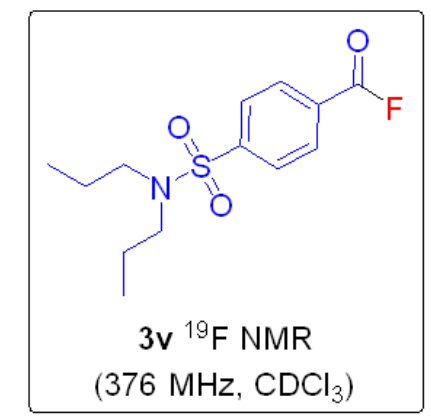

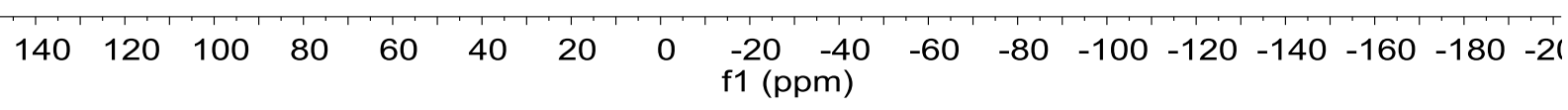

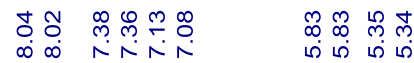

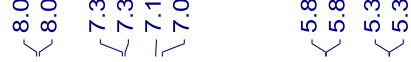

宓

Ti

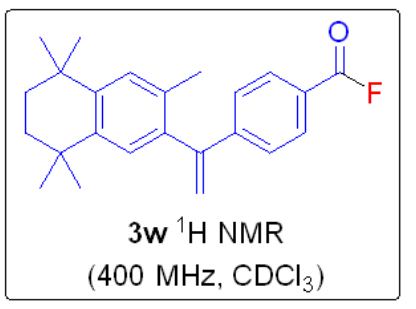

$400 \mathrm{MHz}, \mathrm{CDCl}_{3}$ )

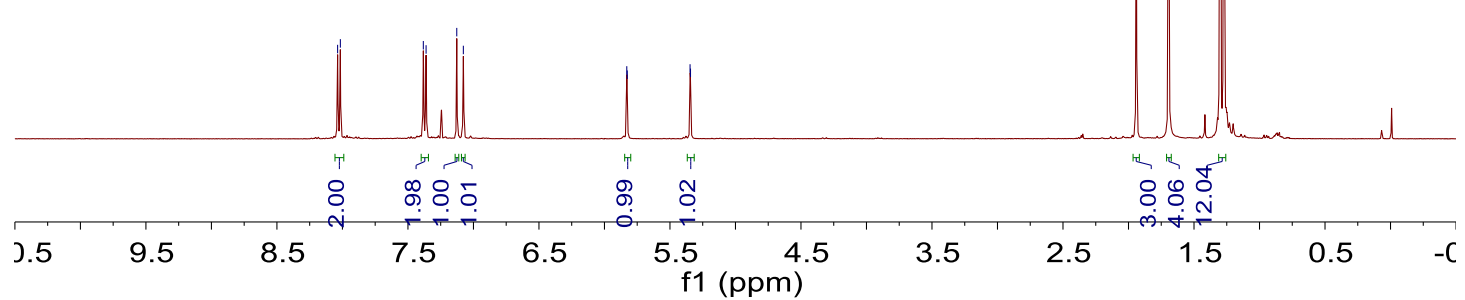




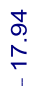

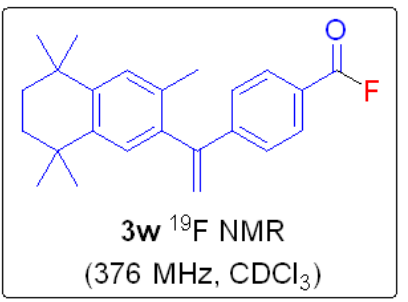

\begin{tabular}{|c|c|c|c|c|c|c|c|c|c|c|c|}
\hline 20 & 0 & -20 & -40 & -60 & $\begin{array}{l}-80 \\
\text { f1 (ppn }\end{array}$ & ר) & -120 & -140 & -160 & -180 & -20 \\
\hline
\end{tabular}
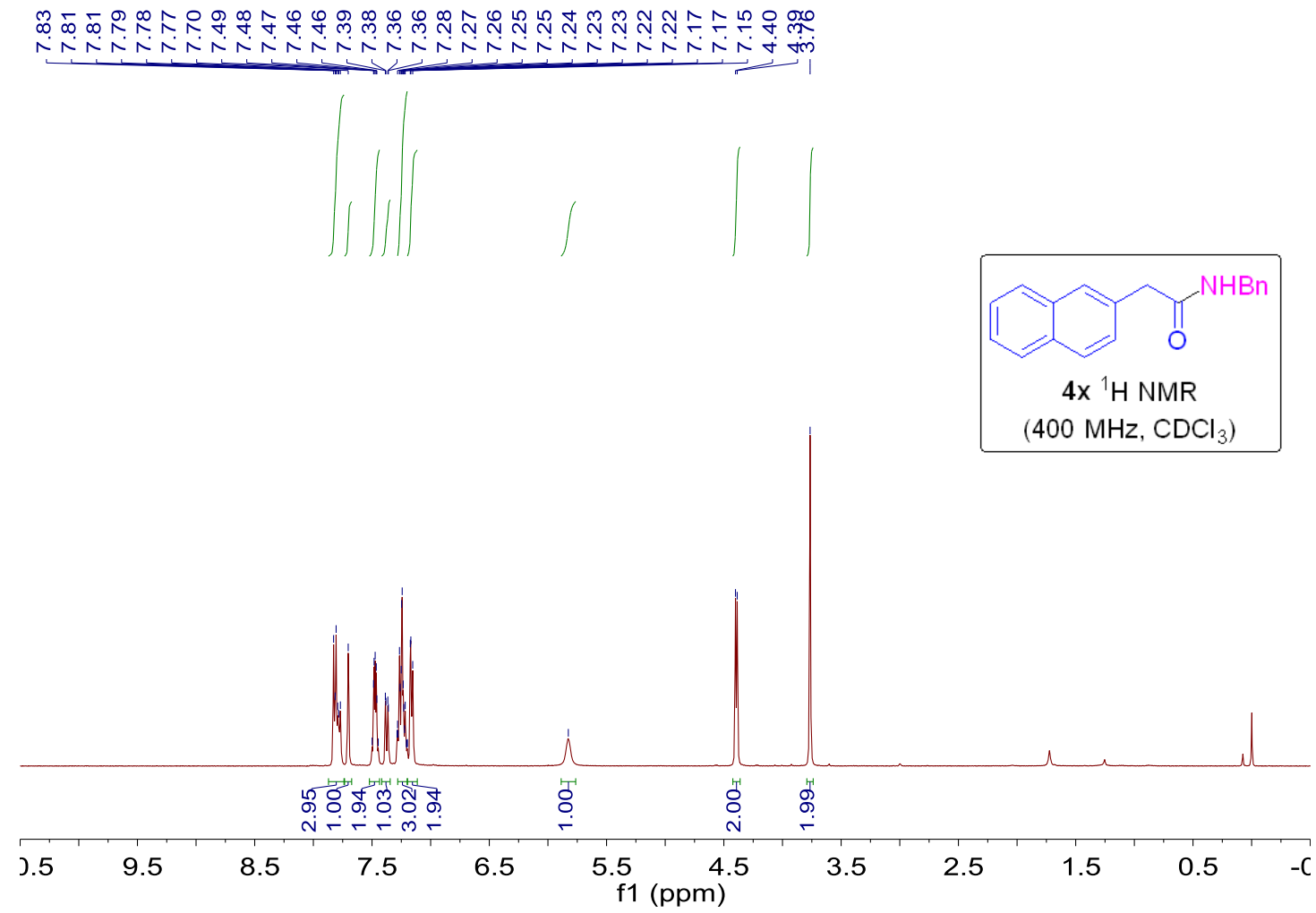

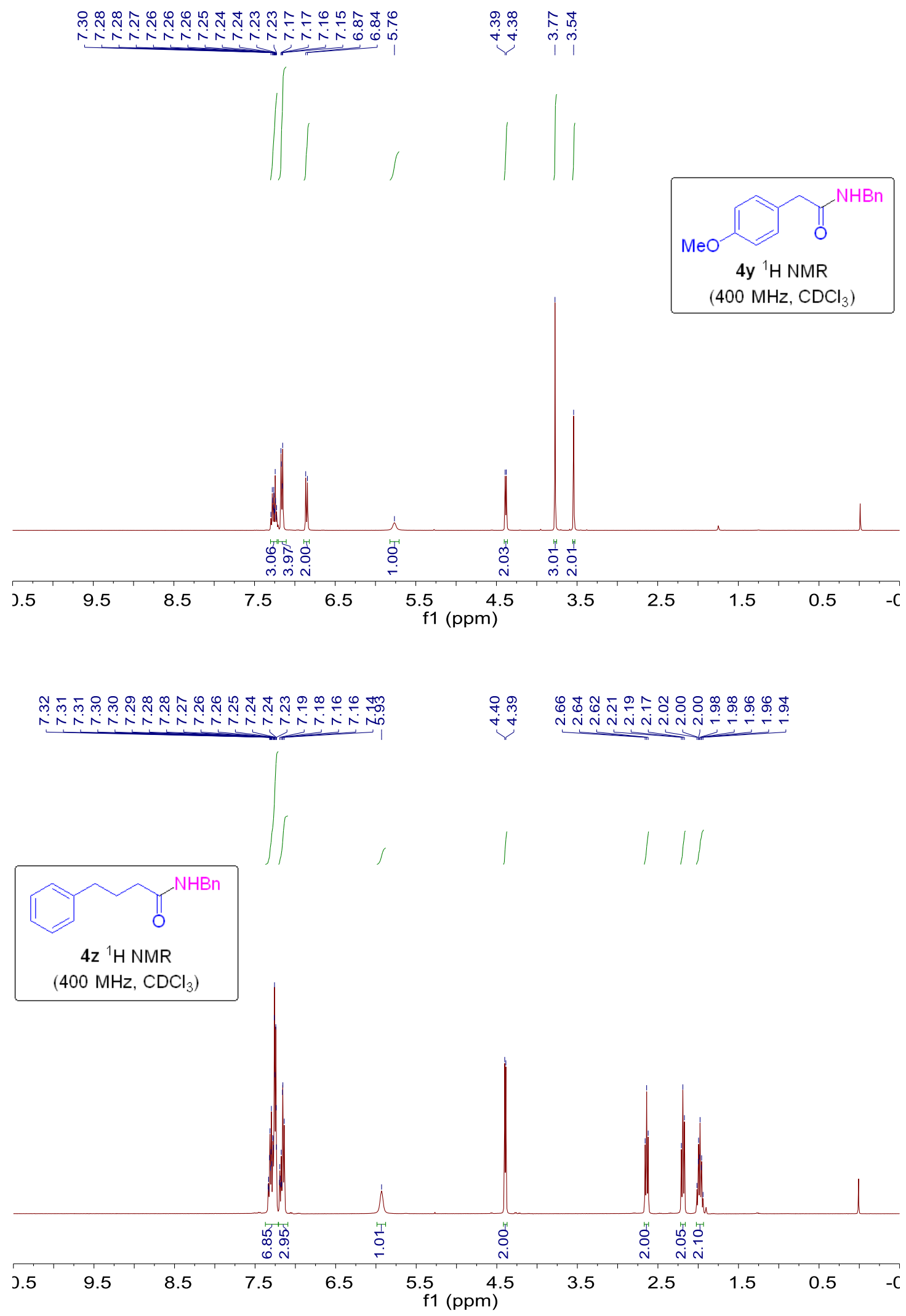


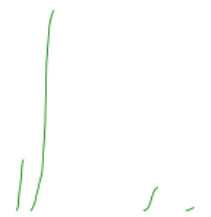

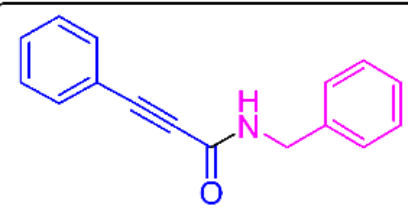

4aa ${ }^{1} \mathrm{H}$ NMR $400 \mathrm{MHZ}, \mathrm{CDCl}_{3}$

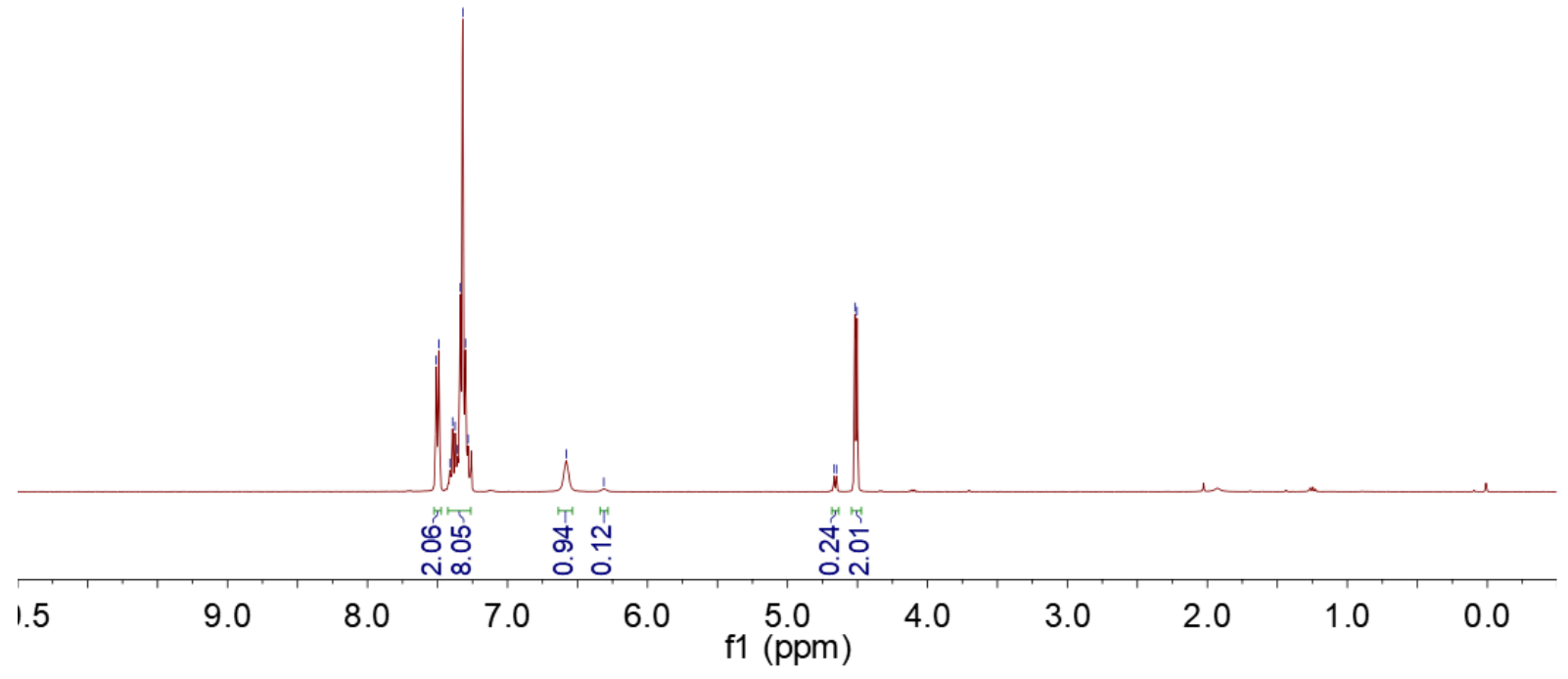

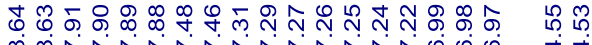
$e_{0}$
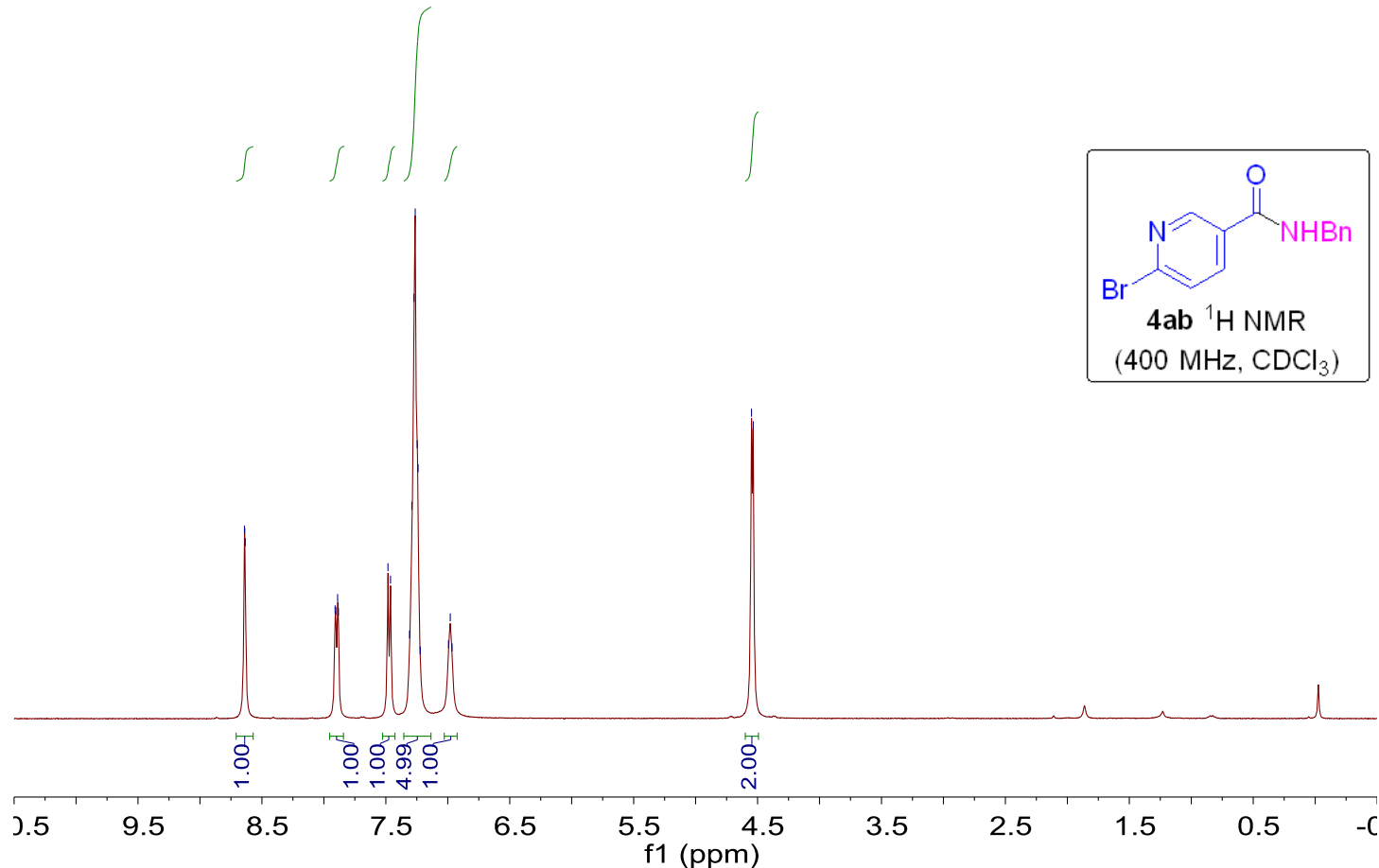

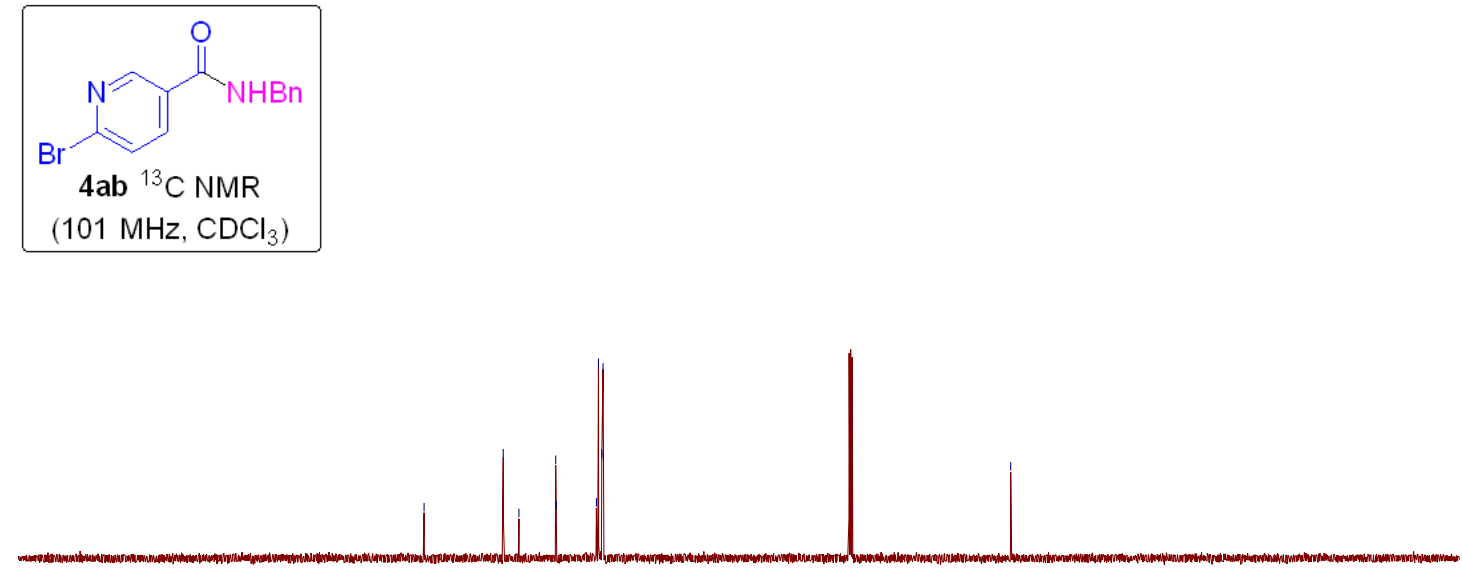

$\begin{array}{llllllllllllllll}240 & 220 & 200 & 180 & 160 & 140 & 120 & \begin{array}{c}100 \\ \mathrm{f} 1(\mathrm{ppm})\end{array} & 80 & 60 & 40 & 20 & 0 & -20 & -40\end{array}$

งุ $ิ$ กุ

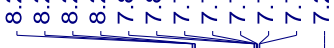
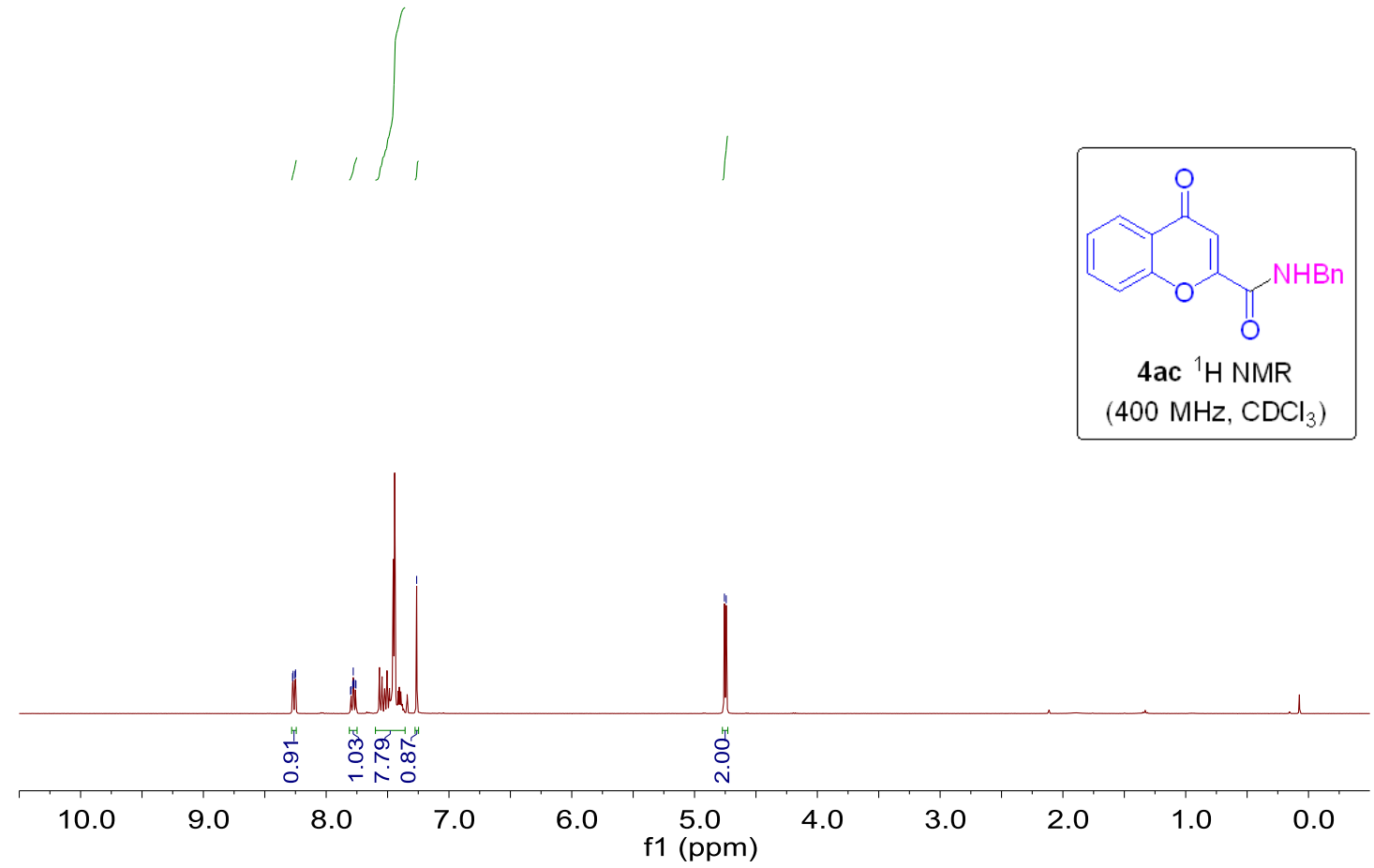


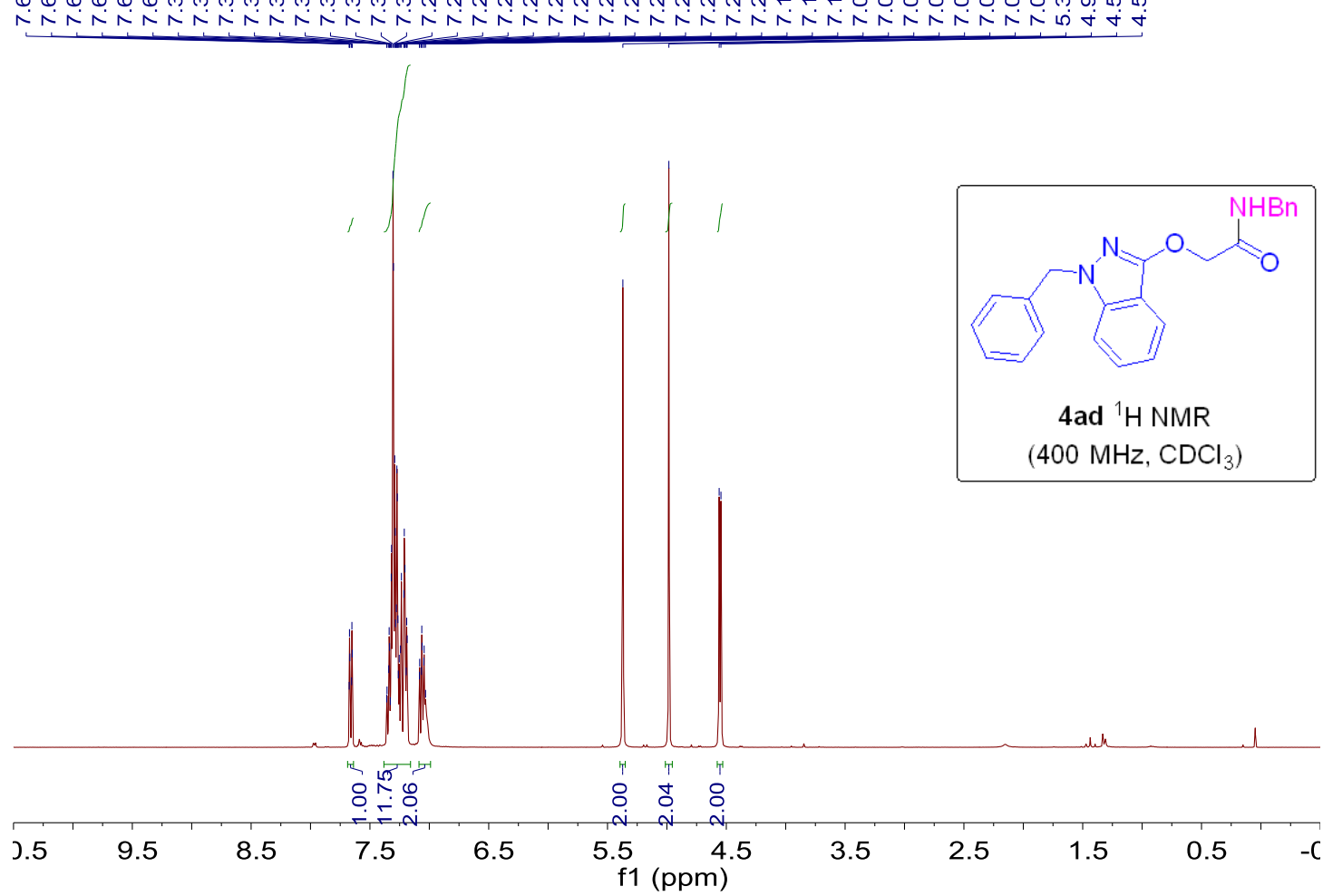

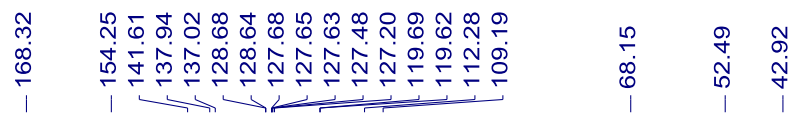

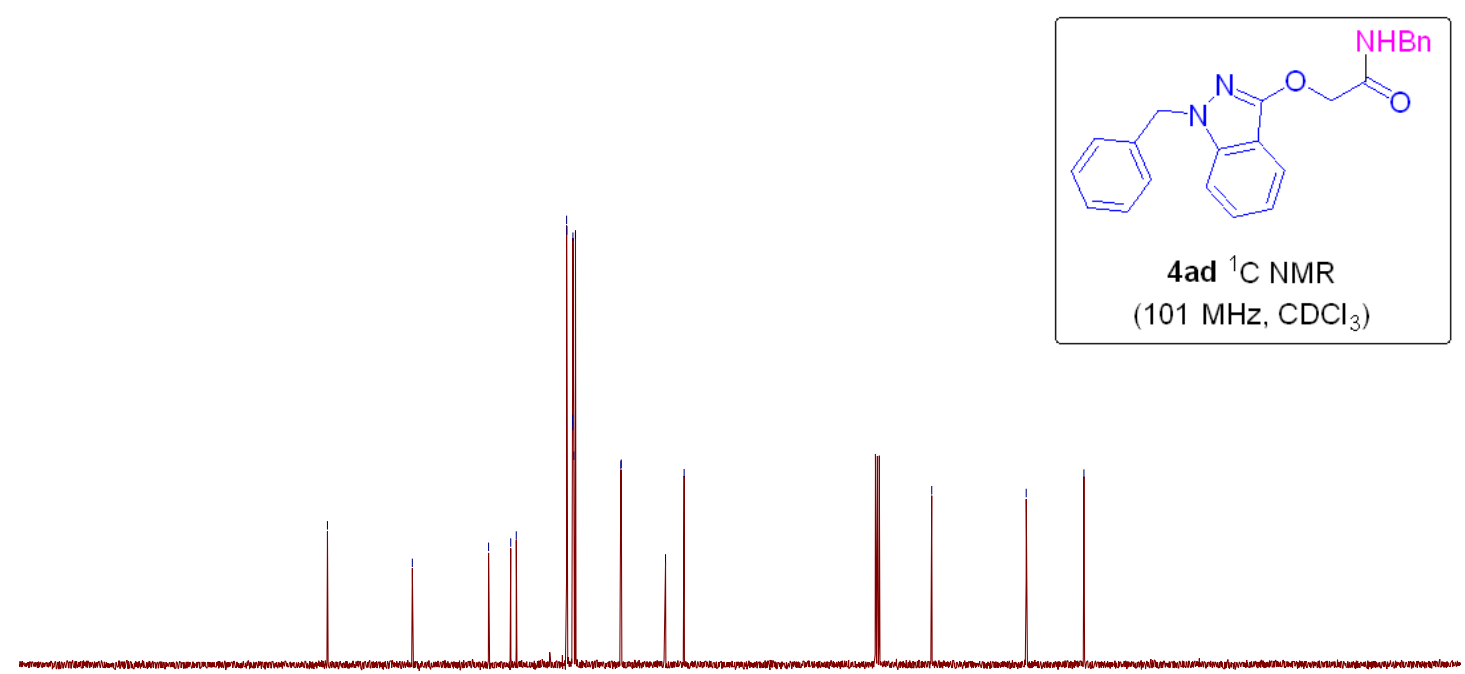

$21020019018017016015014013012011010090 \quad 80 \quad 70 \quad 60 \quad 50 \quad 40 \quad 30 \quad 20 \quad 10 \quad 0 \quad-10$ f1 (ppm) 

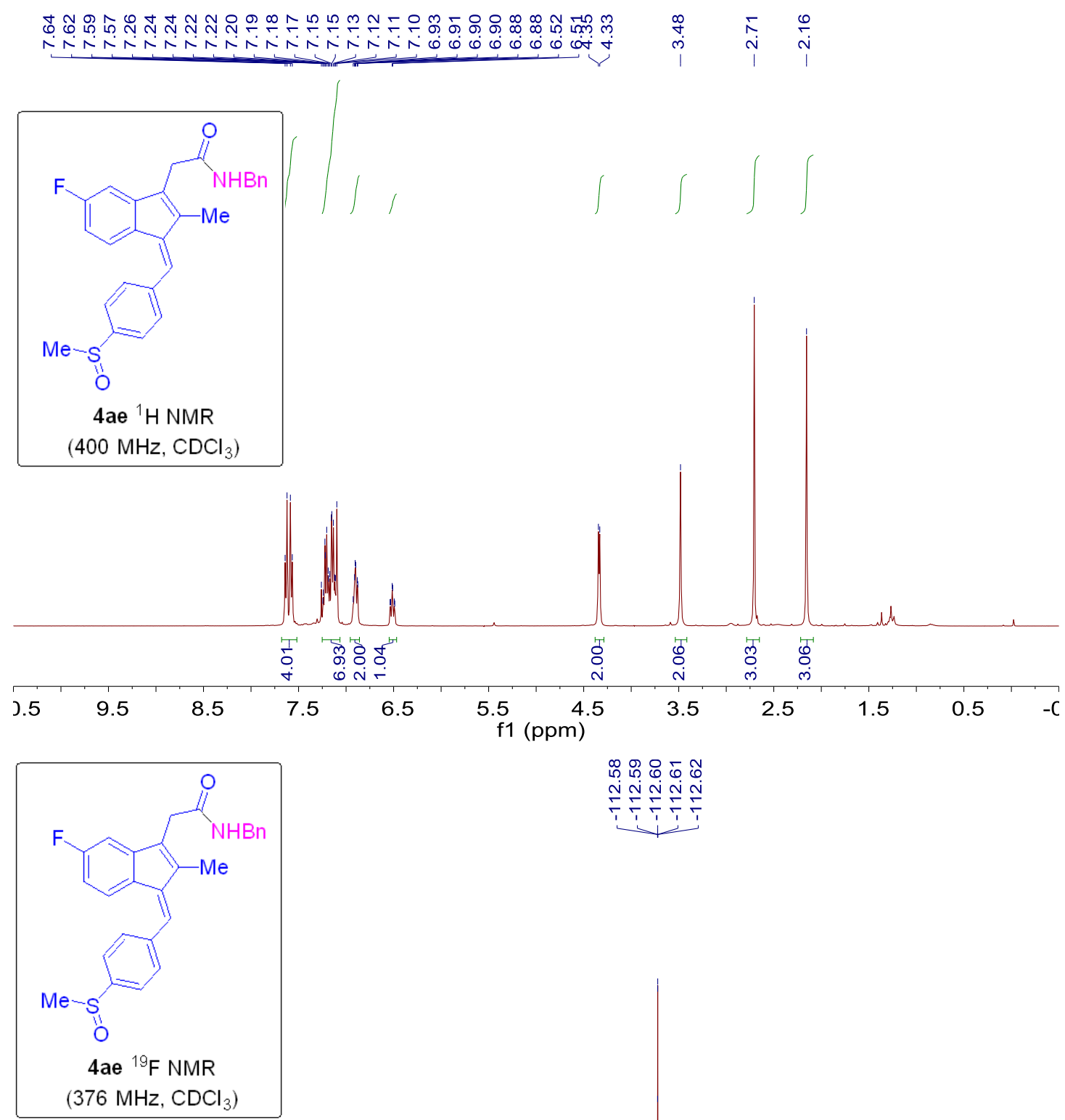

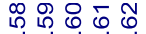

군 $\frac{1}{2} \frac{5}{\mathfrak{N}}$

\begin{tabular}{|c|c|c|c|c|c|c|c|c|c|c|c|}
\hline 20 & 0 & -20 & -40 & -60 & $\begin{array}{l}-80 \\
\text { f1 (ppm }\end{array}$ & -100 & -120 & -140 & -160 & -180 & -20 \\
\hline
\end{tabular}




\section{References}

(1) Deng, X.-Y.; Lin, J.-H.; Zheng, J.; and Xiao, J.-C. Chem. Commun. 2015, 51, 8805-8808.

(2) Meanwell, M.; Lehmann, J.; Eichenberger, M.; Martin, R. E.; Britton, R. Chem. Commun. 2018, 54, 9985-9988.

(3) Wang, Z.; Wang, X.; Nishihara, Y. Chem. Commun. 2018, 54, 13969-13972.

(4) Nongkunsarn, P.; Ramsden, C. A. J. Chem. Soc., Perkin Trans. 1 1995, 121-122.

(5) Scattolin, T.; Deckers, K.; Schoenebeck, F. Org. Lett. 2017, 19, 5740-5743.

(6) Chambers, R. D.; Sandford, G.; Trmcic, J.; Okazoe, T. Org. Process Res. Dev. 2008, 12, 339-344.

(7) Brownlee, R. T. C.; Craik, D. J. Magn Reson Chem. 1980, 14, 187-191.

(8) Baell, J. B.; Duggan, P. J.; Forsyth, S. A.; Lewis, R. J.; Phei Lok, Y.; Schroeder, C. I. Bioorg. Med. Chem. 2004, 12, 4025-4037.

(9) Munoz, S. B.; Dang, H.; Ispizua-Rodriguez, X.; Mathew, T.; Prakash, G. K. S. Org. Lett. 2019, 21, 1659-1663.

(10) Pan, F.-F.; Guo, P.; Li, C.-L.; Su, P.; Shu, X.-Z. Org. Lett. 2019, 21, 3701-3705.

(11) Yang, Z.; Chen, S.; Yang, F.; Zhang, C.; Dou, Y.; Zhou, Q.; Yan, Y.; Tang, L. Eur. J. Org. Chem. 2019, 5998-6002.

(12) Lanigan, R. M.; Starkov, P.; Sheppard, T. D. J. Org. Chem. 2013, 78, 4512-4523.

(13) Métro, T.-X.; Bonnamour, J.; Reidon, T.; Duprez, A.; Sarpoulet, J.; Martinez, J.; Lamaty, Frédéric. Chem. Eur. J. 2015, 21, 12787-12796.

(14) Kawagoe, Y.; Moriyama, K.; Togo, H. Tetrahedron 2013, 69, 3971-3977.

(15) Vydzhak, R. N.; Panchishin, S. Y. Russ. J. Gen. Chem. 2008, 78, 2391-2397.

(16) Mathew, B.; Hobrath, J. V.; Connelly, M. C.; Guy, R. K.; Reynolds, R. C. Bioorg. Med. Chem. Lett. 2017, 27, $4614-4621$. 